Aus der Klinik für Klinische Neurophysiologie

(Prof. Dr. med. W. Paulus)

im Zentrum Neurologische Medizin

der Medizinischen Fakultät der Universität Göttingen

\title{
Untersuchung struktureller zerebraler Alterationen bei Patienten mit idiopathisch-generalisierter Epilepsie unter besonderer Berücksichtigung des Janz-Syndroms
}

\author{
INAUGURAL-DISSERTATION \\ zur Erlangung des Doktorgrades \\ der Medizinischen Fakultät der \\ Georg-August-Universität zu Göttingen
}

vorgelegt von

Christine Diederich

aus

Dortmund

Göttingen 2015 
Dekan:

1. Berichterstatter:

2. Berichterstatter:
Prof. Dr. Heyo K. Kroemer

Prof. Dr. Walter Paulus

PD Dr. Peter Dechent

Tag der mündlichen Prüfung: 07.10.2015 


\section{Inhaltsverzeichnis}

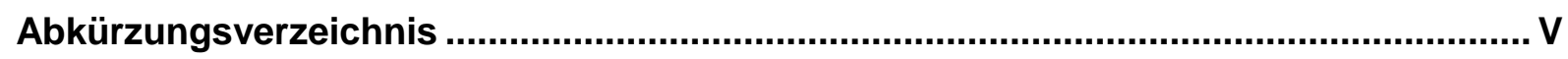

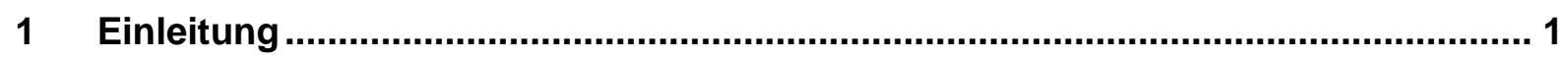

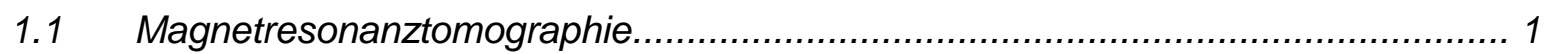

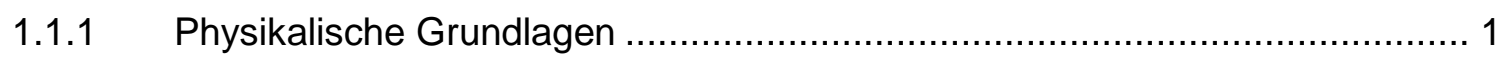

1.1.2 Relaxations-, Repetitions- und Echozeiten …………............................... 2

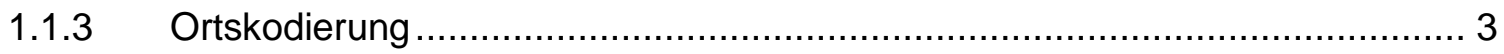

1.1.4 MRT-Techniken und Sequenzen ………………………………….... 3

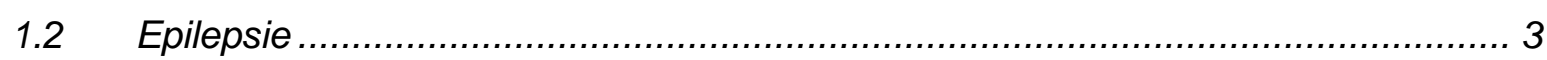

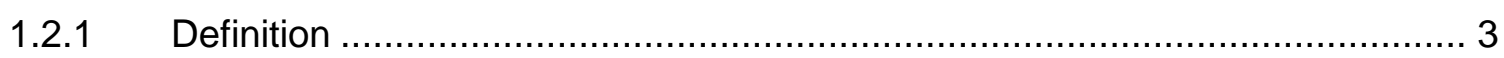

1.2.2 Pathophysiologie und Ätiologie ................................................................. 4

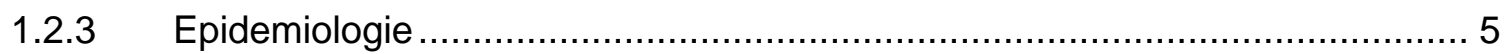

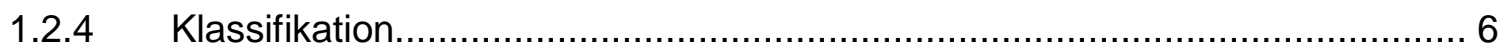

1.2.5 Generalisierte Anfallstypen .................................................................... 7

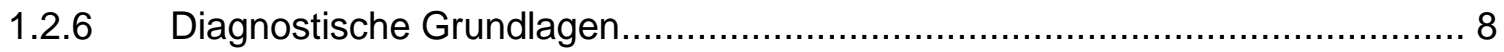

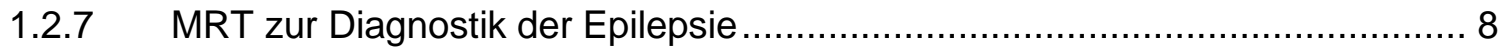

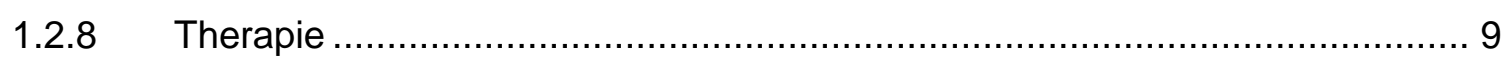

1.3 Bedeutung der MRT-Diagnostik in der Epileptologie .......................................10

$1.4 \quad$ Zielsetzung dieser Studie .........................................................................11

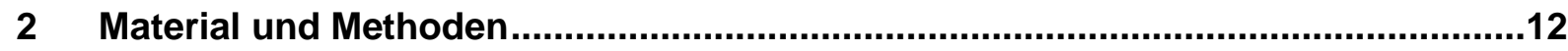

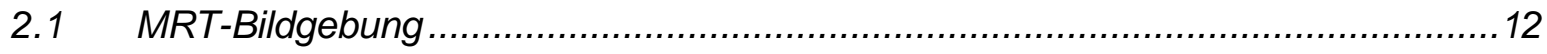

2.1.1 T1-gewichtete Bildgebung ……………………….........................13

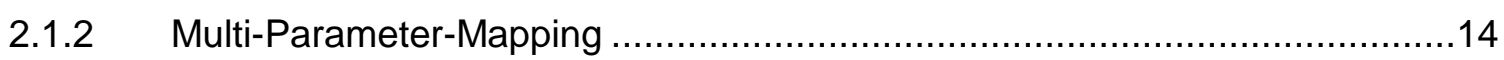

2.1.3 Diffusions-Tensor-gewichtete Bildgebung (DTI) .....................................15

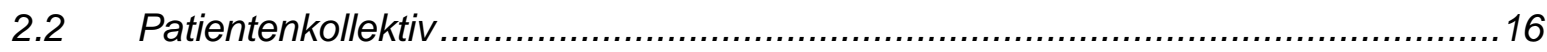

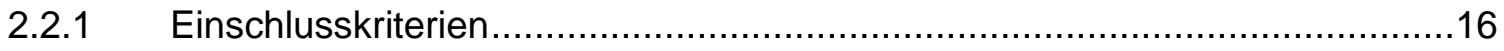

2.2.2 Ausschlusskriterien............................................................................

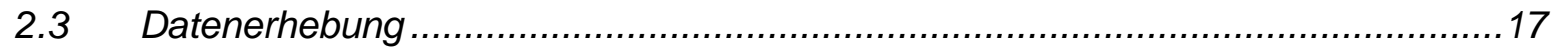

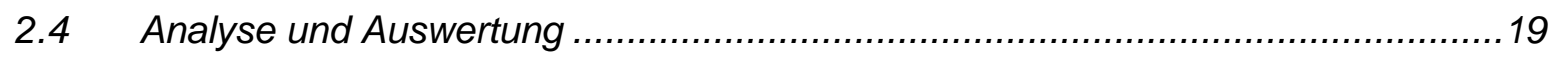

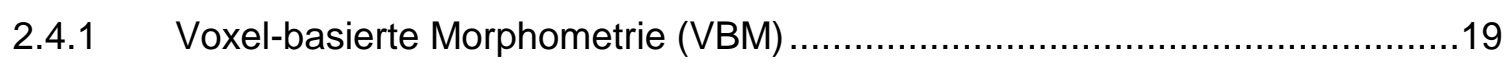

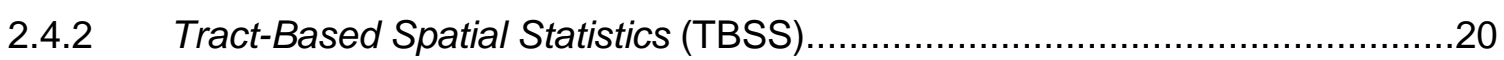


3.1 VBM der T1-gewichteten Sequenz und des Multi-Parameter-Mappings..............22

3.1.1 Vergleich beider Patientengruppen mit der Kontrollgruppe ..........................23

3.1.2 Vergleich der IGE-Patienten mit der Kontrollgruppe...................................27

3.1.3 Vergleich der JME-Patienten mit der Kontrollgruppe...................................31

3.1.4 Vergleich der IGE-Patienten mit den JME-Patienten....................................34

3.1.5 Vergleich der DTI-Daten mit Tract-Based Spatial Statistics ..........................38

3.1.6 Vergleich beider Patientengruppen mit der Kontrollgruppe (FA) ..................39

3.1.7 Vergleich der JME-Patienten mit der Kontrollgruppe (FA) ..........................40

3.1.8 Vergleich der IGE-Patienten mit der Kontrollgruppe (FA) ...........................41

3.1.9 Vergleich der IGE- mit den JME-Patienten (FA) ....................................42

3.1.10 Vergleich beider Patientengruppen mit der Kontrollgruppe (MD) ..................43

3.1.11 Vergleich der JME-Patienten mit der Kontrollgruppe (MD) ............................43

3.1.12 Vergleich der IGE-Patienten mit der Kontrollgruppe (MD) ..........................44

3.1.13 Vergleich der IGE- mit den JME-Patienten (MD) .....................................45

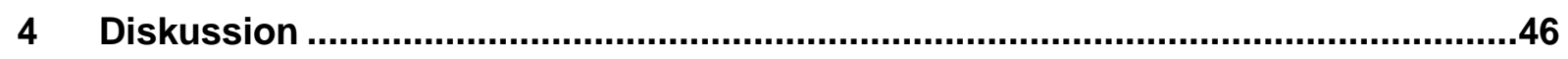

4.1 Veränderungen zerebraler Netzwerke.................................................47

4.2 Strukturelle Alterationen in Verbindung mit Netzwerkveränderungen ...................49

4.3 Epileptogenese und strukturelle Auswirkungen ..........................................51

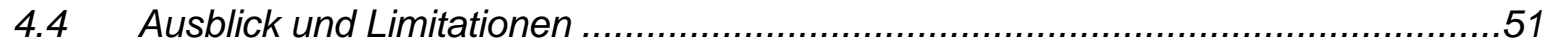

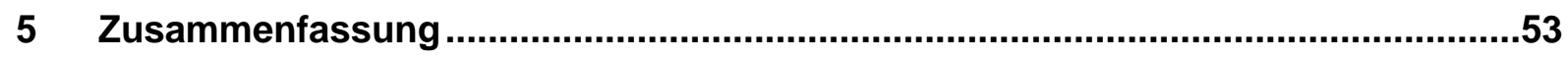

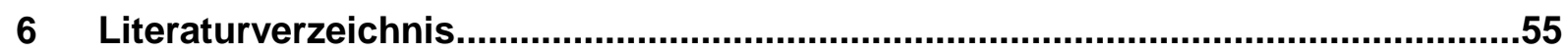

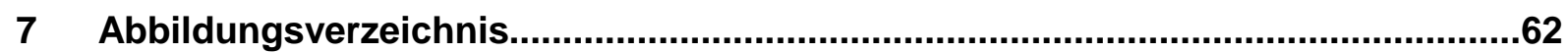

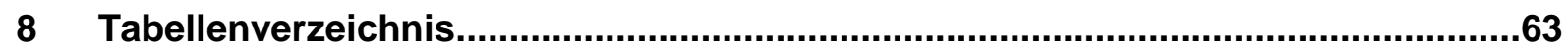

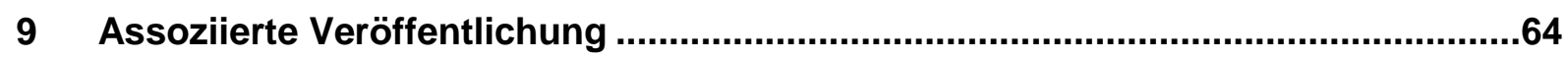

10 Anhang

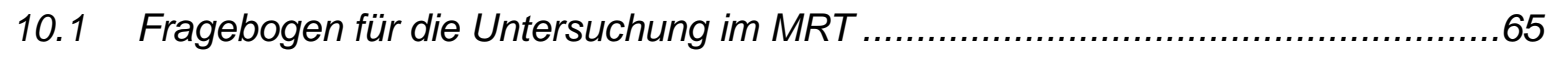

10.2 Aufklärung für die Studienteilnahme................................................68

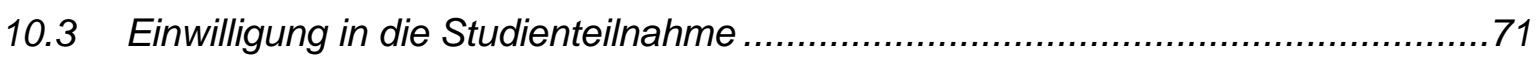




\section{Abkürzungsverzeichnis}

bzw. beziehungsweise

CT Computertomographie

DBS deep brain stimulation, tiefe Hirnstimulation

DTI Diffusions-Tensor-gewichtete Bildgebung (diffusion tensor imaging)

DWI Diffusions-gewichtete Bildgebung (diffusion weighted imaging)

EEG Elektroenzephalographie

EPSP exzitatorisches postsynaptisches Potenzial

FA fraktionelle Anisotropie

FE fokale Epilepsie

FLAIR Fluid Attenuated Inversion Recovery

FMRIB Oxford University Centre for Functional MRI of the Brain

fMRT funktionelle Magnetresonanztomographie

FSL FMRIB Software Library

FWE family wise error

GTKA generalisierte tonisch-klonische Anfälle

HF Hochfrequenz

IGE idiopathisch-generalisierte Epilepsie

ILAE Internationale Liga gegen Epilepsie

IPSP inhibitorisches postsynaptisches Potenzial

JME juvenile myoklonische Epilepsie

MD mittlerer Diffusionskoeffizient

$\mathrm{MNI} \quad$ Montreal Neurological Institute

MR Magnetresonanz

MRT Magnetresonanztomographie

MT Magnetisierungstransfer

MTC Magnetisierungstransferkontrast (magnetization transfer contrast)

MTR Magnetisierungstransferverhältnis (magnetization transfer ratio)

MTRA Medizinisch-Technische(r) Radiologieassistent(in)

PD Protonendichte

PDS paroxysmaler Depolarisationsshift 
PET Positronen-Emissionscomputertomographie

$\mathrm{ROI}$ region of interest

rTMS repetitive transkranielle Magnetstimulation

SPECT Single-Photon-Emissionscomputertomographie

SPM Statistical Parametric Mapping

TBSS Tract-Based Spatial Statistics

tDCS transcranial direct-current stimulation, transkranielle Gleichstromstimulation

TE Echozeit (echo time)

TFCE Threshold-Free Cluster Enhancement

TR Repetitionszeit (repetition time)

UMG Universitätsmedizin Göttingen

usw. und so weiter

VBM Voxel-basierte Morphometrie

ZNS zentrales Nervensystem 


\section{$1 \quad$ Einleitung}

\subsection{Magnetresonanztomographie}

Die Magnetresonanztomographie (MRT) wird seit Anfang der 80er Jahre in der medizinischen Routinediagnostik verwendet (vergleiche Loewenhardt 2006). Sie bietet einen sehr guten Weichteilkontrast sowie eine hohe räumliche Auflösung und wird daher häufig bei Erkrankungen des ZNS eingesetzt. In der Epilepsiediagnostik besitzt die Magnetresonanztomographie einen besonders hohen Stellenwert; sie stellt hier das wichtigste bildgebende Verfahren dar (ILAE Commission Report 1997). Weiter werden bei dieser Technik keine ionisierenden Strahlen verwendet, sodass der Patient keiner Strahlenbelastung ausgesetzt ist.

Als Quellen für die Kapitel 1.1.1-1.1.4 wurde folgende Literatur verwendet: Weishaupt et al. 2009; Loewenhardt 2006; Vlaardingerbroek und den Boer 2004.

\subsubsection{Physikalische Grundlagen}

Die Grundlage der MRT beruht auf dem Kernspineffekt, wobei der Eigendrehimpuls (Spin) von Protonen genutzt wird. Um ein messbares Signal zu erhalten, werden hierfür Atome mit unpaarigen Protonen benötigt. Da der menschliche Körper zu weiten Anteilen aus Wasserstoff besteht und dieser einen großen Resonanzeffekt besitzt, kann er hervorragend in der Magnetresonanztomographie verwendet werden. Dabei entsteht unter Einwirkung eines äußeren Magnetfelds eine Kreiselbewegung um die magnetische Achse des Protons parallel zu den magnetischen Feldlinien (Präzession). Diese Präzessionsbewegung besitzt eine bestimmte Frequenz (Lamorfrequenz) und ist von der Stärke des Magnetfelds und dem gyromagnetischen Faktor des entsprechenden Elements abhängig.

Unter normalen Umständen liegt eine stochastische Verteilung der magnetischen Dipolmomente im Raum vor. Durch den supraleitenden Magneten des Magnetresonanztomographen entsteht ein starkes Magnetfeld (hier drei Tesla), sodass die magnetischen Momente parallel und antiparallel ausgerichtet werden. Dieses wird als Spin-up bzw. Spindown bezeichnet. Aufgrund der energetisch günstigeren Spin-up-Ausrichtung, in der sich etwas mehr Protonen befinden als in der Spin-down-Ausrichtung, entsteht so eine Nettomagnetisierung parallel zum äußeren Magnetfeld (z-Richtung), die für den Resonanzeffekt und damit für die Bildgebung genutzt werden kann. Dies geschieht, indem durch eine Hochfrequenzspule ein Hochfrequenzimpuls (HF-Impuls) mit der entsprechenden 
Energie und Lamorfrequenz in den Körper eingebracht wird. Durch diese Anregung des Spinsystems wird die Längsmagnetisierung $M_{z}$ in die $x y$-Ebene überführt und die Protonen präzedieren in Phase. Nach der Beendigung des HF-Impulses kehrt das Spinsystem wieder in den vorherigen Zustand zurück, d.h. die Magnetisierung in Richtung der xy-Ebene nimmt ab. Die dadurch freigesetzte Energie wird in der Empfangsspule aufgenommen und dort ein elektrischer Strom, das Magnetresonanzsignal (MR-Signal), induziert.

\subsubsection{Relaxations-, Repetitions- und Echozeiten}

Nach Beendigung des Impulses kehren die Protonen in ihren Grundzustand zurück, es beginnen die Längs- sowie die Querrelaxation. Bei der Längsrelaxation wird Energie an benachbarte Moleküle abgegeben (Spin-Gitter-Relaxation) und die Längsmagnetisierung $M_{z}$ in Richtung des Hauptmagnetfeldes wieder hergestellt. Dies geschieht in der charakteristischen Relaxationszeit T1, in der 63,2 \% der vorherigen Längsmagnetisierung wieder aufgebaut wurden. Ebenso beginnen die Spins der Protonen mit der Zerfallskonstante T2 zu dephasieren (Spin-Spin-Relaxation), und die transversale Magnetisierung bildet sich zurück. Da die Spins sich nicht nur gegenseitig beeinflussen und so desynchronisieren, sondern dies durch Inhomogenitäten des äußeren Magnetfeldes zusätzlich gefördert wird, wird das Signal mit der Zeitkonstanten T2* verkleinert.

Zur MR-Bildgebung muss die entsprechende Schicht sehr häufig angeregt werden. Dabei wird die Zeit zwischen den Anregungen als Repetitionszeit (TR) bezeichnet. Die Größe der TR bestimmt den Einfluss von T1 auf den Bildkontrast. Gewebe mit einer langen T1 können bei einer kurzen TR nicht ausreichend zwischen den einzelnen Anregungen relaxieren und geben so nur wenig Signal ab. Sie stellen sich im entsprechenden Bild hypointens dar. Der Kontrast zwischen diesen Geweben und denen, die eine kurze T1-Relaxationszeit besitzen und mehr Signal abgeben, wird also besonders deutlich (hohe T1-Gewichtung). Bei einer langen TR kann die Längsmagnetisierung annähernd überall wieder aufgebaut werden, wodurch die Signalabgabe generell zunimmt. Es ist also kein spezifischer Unterschied mehr zwischen verschiedenen Strukturen ersichtlich. Der T1-Kontrast sowie die T1-Gewichtung nehmen folglich ab.

Als Echozeit (TE) wird die Zeit bezeichnet, die zwischen der Anregung des Gewebes und der Aufnahme des entsprechenden Signals liegt. Bei einer kurzen TE hat die Dephasierung gerade erst angefangen, und die Bedeutung von T2 auf den Kontrast der unterschiedlichen Gewebe ist niedrig (geringe T2-Gewichtung). Bei längeren Echozeiten sind maßgeblich die Gewebe mit langer T2 für das MR-Signal von Bedeutung, sodass hier von einer T2Gewichtung gesprochen wird. 


\subsubsection{Ortskodierung}

Zu einem MRT gehören weiterhin Gradientenspulen, die eine räumliche Kodierung erlauben. Um eine spezielle Schicht darzustellen, wird der Effekt ausgenutzt, dass Anregungs- und Lamorfrequenz für den Resonanzeffekt übereinstimmen müssen. Da die Lamorfrequenz von der Stärke des Magnetfeldes abhängig ist, können Inhomogenitäten des Magnetfeldes dazu verwendet werden, dass nur eine Schicht selektiv angeregt werden kann. Hierzu wird entlang der z-Richtung, also entlang der longitudinalen Körperachse der zu untersuchenden Person, durch eine Magnetspule ein Gradient aufgebaut. Je stärker der Gradient ist, desto dünner sind die anregbaren Schichten.

Für die genaue Ortskodierung wird zusätzlich ein Gradient entlang der y-Achse aufgebaut, sodass die Spins im oberen Teil des Körpers etwas schneller präzedieren als im unteren Teil. Die Lokalisation findet also aufgrund der unterschiedlichen Phasen statt (Phasenkodierung). Durch eine Frequenzkodierung in Abhängigkeit von der Stärke des Magnetfeldes entlang der x-Achse (zum Beispiel rechts stärker als links) findet zusätzlich eine Ortskodierung statt, sodass jedes Voxel durch eine bestimmte Phase und Frequenz gekennzeichnet ist. Das empfangene Signal wird anschließend mittels einer FourierTransformation umgerechnet und mit diesen Daten das MR-Bild erzeugt.

\subsubsection{MRT-Techniken und Sequenzen}

Die Magnetresonanztomographie bietet ein breites Spektrum unterschiedlicher Techniken. Je nach diagnostischer Fragestellung können ganz verschiedene Methoden und Sequenzen, wie zum Beispiel T1, T2- oder Diffusions-Tensor-gewichtete Bildgebung (diffusion tensor imaging, DTI), quantitative Magnetisierungstransfer-Karten, Magnetresonanz-Angiographie oder funktionelle Magnetresonanztomographie (fMRT) eingesetzt werden.

Eine ausführliche Beschreibung zu den in dieser Arbeit verwendeten Methoden findet sich in Kapitel 2.1.

\section{$1.2 \quad$ Epilepsie}

\subsubsection{Definition}

Epilepsie steht als Oberbegriff für diverse Funktionsstörungen des Gehirns, die durch eine überdurchschnittlich hohe Prädisposition für rekurrente und unvorhersehbare Unterbrechungen der normalen Gehirnaktivität charakterisiert sind (Banerjee et al. 2009; Fisher et al. 2005). Diese werden als epileptische Anfälle bezeichnet und gehen mit oder ohne paroxysmale Erscheinungen des vegetativen Nervensystems, der Motorik bzw. der 
Sensibilität und/oder Bewusstseinsbeeinträchtigungen einher (Mattle und Mumenthaler 2013).

Um von einer manifesten Epilepsie zu sprechen, muss mindestens ein epileptischer Anfall vorliegen. Die alleinige erhöhte Prädisposition, zum Beispiel aufgrund von Epilepsiemustern im Elektroenzephalogramm (EEG), ist nicht ausreichend, um eine Epilepsie zu determinieren (Fisher et al. 2005). Zusätzlich sollte eine andauernde strukturelle oder funktionelle Veränderung des Gehirns nachweisbar sein, durch die das erneute Auftreten von epileptischen Anfällen begünstigt wird (Engel 2006). In der Praxis wird auch von einer erhöhten Prädisposition ausgegangen, wenn zwei unprovozierte Anfälle mit einem zeitlichen Abstand von mindestens 24 Stunden stattgefunden haben. Ebenso empfiehlt die Internationale Liga gegen Epilepsie auch von einer manifesten Epilepsie zu sprechen, wenn mindestens zwei Anfälle im Rahmen einer Reflexepilepsie aufgetreten sind (Fisher et al. 2013).

Zu dieser Erkrankung gehört häufig neben den epileptischen Anfällen ein deutlicher Verlust der Lebensqualität, wie zum Beispiel der Entzug der Fahrerlaubnis oder eine eingeschränkte Berufsfähigkeit (Austin et al. 2008; Jacoby et al. 2008). Die sozialen, kognitiven und psychologischen Konsequenzen des Anfallsleidens, wie beispielsweise Gedächtnisstörungen oder postiktale Verwirrtheitszustände, können darüber hinaus mit einer Stigmatisierung, dem Ausschluss aus dem öffentlichen Leben und mit einer sozialen Isolation einhergehen und sollten bei der Therapie bedacht werden (Jacoby et al. 2008; Fisher et al. 2005).

\subsubsection{Pathophysiologie und Ätiologie}

Der Erkrankung Epilepsie können je nach Epilepsieform (symptomatisch, idiopathisch oder kryptogen) unterschiedliche Ursachen zugrunde liegen (vergleiche für diesen Abschnitt: Masuhr et al. 2013; Hacke 2010; Tettenborn et al. 2006). Bei der idiopathischen Form der Epilepsie lassen sich in der Routine-Bildgebung keine ursächlichen Befunde darstellen. Es findet sich jedoch häufig eine familiäre Disposition, die durch genetische Alterationen von Membrankanälen oder Transmitterrezeptoren gekennzeichnet ist oder diese vermutet werden. Bei der symptomatischen Epilepsie ist das Anfallsleiden Symptom einer anderen Erkrankung, wie zum Beispiel eines Tumors, einer Enzephalitis oder vaskulärer Erkrankungen. Ebenso kann eine Epilepsie durch zerebrale Entwicklungsstörungen und perinatale Komplikationen entstehen oder als Folge eines Schädel-Hirn-Traumas. Bei kryptogener Epilepsie kann mit aktuellen diagnostischen Verfahren keine Ursache für die 
Entstehung der epileptischen Anfälle gefunden werden, es wird jedoch eine fokale Genese vermutet.

Pathophysiologisch lässt sich Epilepsie auf eine gestörte Funktion bzw. Kommunikation von Neuronen bzw. Neuronenverbänden zurückführen, die durch ein Ungleichgewicht von inhibitorischen und exzitatorischen postsynaptischen Potenzialen (IPSP und EPSP) hervorgerufen werden kann (McCormick und Contreras 2001; Johnston und Brown 1984). Bei diesem paroxysmalen Depolarisationsshift (PDS) kommt es über eine starke Depolarisation von Membranen zu salvenartigen Aktionspotenzialen (Sankar und Rho 2009; Dichter 2008). Terminiert wird der Vorgang durch eine inhibierende Hyperpolarisation. Aufgrund der engen Vernetzung von Neuronen können davon ganze Nervenzellverbände betroffen sein, was sich diagnostisch im EEG als Spikes bzw. Sharp Waves manifestiert (Mattle und Mumenthaler 2013). Diese Einbeziehung weiterer Nervenzellen kann auf lokaler Ebene stattfinden, ebenso kann sie entfernte, kontralaterale und subkortikale Regionen erreichen. Besonders anfällig hierfür sind bei fokaler Epilepsie das limbische System im Temporallappen, der Hippocampus, der entorhinale Kortex sowie die Amygdala bzw. thalamokortikale Netzwerke bei generalisierten Epilepsien (Dichter 2008; Blumenfeld 2003).

\subsubsection{Epidemiologie}

Epilepsie gehört zu den ältesten und häufigsten bekannten Erkrankungen der Menschheit, die sich über alle Altersklassen erstreckt (Banerjee et al. 2009). Die Prävalenz liegt bei ca. 0,45 - 0,7 \% in der europäischen Bevölkerung, wobei weltweit auch höhere Zahlen gemessen wurden (Forsgren et al. 2005). Diese variieren aufgrund verschiedener Faktoren, wie zum Beispiel Zugangsmöglichkeiten zur medizinischen Versorgung und werden mit Werten von bis zu $2 \%$ beschrieben (Banerjee et al. 2009).

Die Anzahl der Neuerkrankungen pro Jahr ist stark von der Altersklasse abhängig. Die Inzidenz bei Kindern und Jugendlichen liegt bei 0,05 \% und die der 20 - 64-Jährigen bei 0,03\% (Forsgren et al. 2005). Bei Erwachsenen ab dem 65. Lebensjahr ist ein erneuter Anstieg auf bis zu 0,1 \% zu verzeichnen (Forsgren et al. 2005). Die meisten Neuerkrankungen treten somit im Kindes- und Säuglingsalter sowie ab dem 65. Lebensjahr auf.

Die Wahrscheinlichkeit, an Epilepsie zu erkranken, steigt je nach Epilepsieform deutlich an, wenn bereits Familienmitglieder betroffen sind. Ist ein Elternteil zum Beispiel an einer idiopathischen Epilepsie erkrankt, so erhöht sich das Risiko für das Kind auf etwa $4 \%$ 
(Hacke 2010). Dabei liegt das Erkrankungsrisiko für Verwandte ersten und zweiten Grades bei generalisierten Formen deutlich über dem Risiko für Nachkommen von Patienten mit einer fokalen Epilepsie (Abou-Khalil et al. 2007).

\subsubsection{Klassifikation}

Es gibt multiple Formen von Epilepsie, die anhand verschiedener Gesichtspunkte klassifiziert werden können. Zu diesen Eigenschaften zählen die Anfallsformen, epileptische Syndrome, ätiologische Aspekte sowie weitere Konzepte, zum Beispiel das Alter der Erstmanifestation. Aufgrund neuer Erkenntnisse werden diese Klassifikationen wiederholt von der Kommission für Klassifikation und Terminologie der Internationalen Liga gegen Epilepsie (ILAE) überarbeitet und verschiedene Änderungen vorgeschlagen. Insbesondere ätiologische Aspekte rücken hier in den Vordergrund (Berg et al. 2010).

Zurzeit wird bei der Kategorisierung der Anfallsformen im klinischen Altag weiterhin die Klassifikation von 1981 verwendet (Commission on Classification and Terminology of the International League Against Epilepsy 1981). Diese sieht eine Unterscheidung zwischen generalisierten, fokalen/lokalisationsbezogenen sowie nicht klassifizierbaren Anfällen vor. Epilepsien bzw. Syndrome werden weiter in die Kategorien idiopathisch, symptomatisch oder kryptogen unterteilt, wobei die ILAE mittlerweile die Bezeichnungen "genetisch“, „strukturell/metabolisch“ und „unbekannt“ hierbei befürwortet (Berg et al. 2010). Aufgrund der klinischen Etablierung der Einteilung von 1981 sowie ihrer Verwendung in den meisten wissenschaftlichen Arbeiten wurde diese auch hier zugrunde gelegt.

Die Gruppe der idiopathisch-generalisierten Epilepsien (IGE) enthält verschiedene Syndrome, bei denen eine genetische Ätiologie vermutet wird oder bekannt ist. Zu diesen Syndromen zählen juvenile myoklonische Epilepsien (Janz-Syndrom, JME), benigne familiäre Neugeborenenkrämpfe, benigne Neugeborenenkrämpfe, benigne myoklonische Epilepsien des Kleinkindalters, Absence-Epilepsien des Kindesalters, juvenile AbsenceEpilepsien und Aufwach-Grand-mal-Epilepsien (Mattson 2003).

Die juvenile myoklonische Epilepsie (auch Janz-Syndrom) ist durch meist bilaterale myoklonische Anfälle gekennzeichnet, die mit tonisch-klonischen Anfällen bzw. Absencen einhergehen können. Die Myoklonien treten meist erstmalig im Jugendalter bzw. in der Adoleszenz auf und äußern sich vor allem in der oberen Extremität. Sie finden zudem gehäuft morgens nach dem Aufstehen oder bei Schlafentzug (Provokationsfaktor) statt. In vielen Fällen lässt sich eine familiäre Prädisposition feststellen (sie oben). Da Absencen und 
Myoklonien aufgrund ihrer kurzen Dauer und Frequenz häufig nicht bemerkt werden, ist die erste bewusste Manifestation in vielen Fällen ein tonisch-klonischer Anfall. Therapeutisch können die Patienten meist gut medikamentös eingestellt werden, wobei sich die Rezidivgefahr nach Absetzen der Medikamente als relativ hoch erwiesen hat (Tettenborn et al. 2006; Mattson 2003; Fröscher et al. 2004).

\subsubsection{Generalisierte Anfallstypen}

Die ILAE benennt sechs verschiedene generalisierte Anfallstypen, von denen die Absencen sowie die myoklonischen und tonisch-klonischen Anfälle typischerweise bei Patienten mit einer idiopathisch-generalisierten Epilepsie auftreten (vergleiche für diesen Abschnitt: Masuhr et al. 2013; Mattle und Mumenthaler 2013; Hacke 2010; Fusco et al. 2008; Tettenborn et al. 2006; Mattson 2003).

Absencen, auch Petit-Mal genannt, sind ca. 3 - 15 Sekunden dauernde Bewusstseinsstörungen, die in der Regel plötzlich beginnen und ebenso wieder enden. Sie zeigen charakteristische 3/s Spike-Wave-Muster im EEG und treten vor allem im Kindesalter auf. Es kann zu diskreten motorischen Veränderungen, wie zum Beispiel Lidflattern kommen, wobei generell keine Tonusanomalien bei typischen Absencen zu erwarten sind. Treten diese auf oder liegen autonome Störungen bzw. kein abrupter Beginn oder abruptes Ende der Absence vor, sind diese Absencen als atypisch zu bewerten.

Myoklonische Anfälle sind durch kurze schnelle Muskelzuckungen gekennzeichnet, die häufig symmetrisch auf beiden Körperseiten. Sie beginnen typischerweise in der Adoleszenz und persistieren im Erwachsenenalter. Charakteristisch zeichnen sie sich durch das Auftreten in den frühen Morgenstunden nach dem Aufstehen aus und stellen sich im iktalen EEG als irreguläre Epilepsiemuster dar.

Generalisierte tonisch-klonische Anfälle (GTKA, Grand-mal) treten bei Gelegenheitsanfällen, primär generalisierten oder fokalen Epilepsien mit sekundärer Generalisierung auf. Sie können mit Absencen und/oder myoklonischen Anfällen einhergehen und sich durch vorherige Prodromi (zum Beispiel Kopfschmerzen, Übelkeit) oder Auren ankündigen, wobei Auren auf eine fokale Entstehung rückschließen lassen. GTKA beginnen teilweise mit einem Initialschrei, der durch die gleichzeitige Kontraktion von Atem- und Kehlkopfmuskulatur entsteht, sodass die Luft durch eine (fast) verschlossene Stimmritze gepresst wird. Darauf folgt eine tonische Phase, die durch Bewusstseinsverlust und eine tonische Beuge- oder Streckhaltung gekennzeichnet ist. Durch einen plötzlichen Schluss des Mundes kann es weiter zu intraoralen Verletzungen kommen. Zudem kann durch die Kontraktion der Atemmuskulatur eine Apnoe entstehen. Es folgen generalisierte Kloni und der GTKA endet im Terminalschlaf (Minuten bis Stunden). Im EEG zeigt sich vor Beginn des Anfalls häufig 
eine Abflachung, an die sich iktal hochfrequente und hochamplitude Spikes mit Bewegungsartefakten anschließen. Postiktal fällt oft ein verlangsamter Grundrhythmus auf. Tonische, klonische und atonische Anfälle beziehen sich immer auf eine iktale Veränderung der Muskelaktivität. Bei tonischen Anfällen ist diese gesteigert und geht meistens mit einer Bewusstseinsstörung einher, ohne dass klonische Merkmale erkennbar sind. Ausschließlich klonische Anfälle finden sich vor allem bei Neugeborenen oder Kleinkindern und manifestieren sich durch asymmetrische Muskelkontraktionen, die irregulär erscheinen. Wird der Muskeltonus während eines Anfalls merklich verringert, ist dies ein charakteristisches Zeichen für einen atonischen Anfall. Bei einer stärkeren Ausprägung kann es dabei zum Beispiel zu einem Tonusverlust in der unteren Extremität mit einem Sturzereignis kommen.

\subsubsection{Diagnostische Grundlagen}

Die Diagnostik in der Epilepsie dient vor allem der Fragestellung, ob eine manifeste Erkrankung vorliegt sowie der möglichen Eruierung des Anfallstyps und der ätiologischen Aspekte (vergleiche Deutsche Gesellschaft für Neurologie 2013: S1-Leitlinie „Erster epileptischer Anfall und Epilepsien im Erwachsenenalter“; Masuhr et al. 2013,). Neben einer ausführlichen Anamnese (inklusive Fremdanamnese) sowie einer Blut- und/oder Liquoruntersuchung zum Ausschluss infektiöser, metabolischer oder toxischer Ursachen kommt dem EEG (mit/ohne Videoüberwachung) und der bildgebenden Diagnostik, insbesondere der Magnetresonanztomographie, eine zentrale Bedeutung zu (Jackson und Kuzniecky 2008; ILAE Commission Report 1997). Ist die Ursache der Anfälle weiterhin unbekannt, kann eine Positronen-Emissionscomputertomographie (PET) bzw. eine SinglePhoton-Emissionscomputertomographie (SPECT) folgen.

\subsubsection{MRT zur Diagnostik der Epilepsie}

Das MRT hat aufgrund des guten Weichteilkontrasts einen hohen Stellenwert in der Epilepsiediagnostik. Es sollte daher bei jedem Patienten mit Verdacht auf Epilepsie ein strukturelles MRT durchgeführt werden, insbesondere bei Patienten mit fokaler oder sekundär-generalisierter Epilepsie sowie bei Epilepsie-Patienten, die progrediente neurologische oder neuropsychologische Störungen entwickeln (ILAE Neuroimaging Commission 1997).

Vor allem bei symptomatischen Epilepsien können bestimmte Ursachen, wie zum Beispiel Tumore, kortikale Dysplasien, Hippocampussklerosen, zerebrale Ischämien und Blutungen im MRT gut dargestellt werden (Masuhr et al. 2013). Eine weitere wichtige Indikation ist eine Pharmakoresistenz bzw. eine mit Medikamenten nicht zufriedenstellende Anfallssituation. Hier kann eine chirurgische Therapie die einzige Alternative darstellen, bei der die 
Möglichkeit einer Anfallsfreiheit oder -reduktion besteht (Wiebe et al. 2001). Die Lokalisation eines epileptogenen Fokus stellt dabei einen wichtigen Faktor für eine erfolgreiche Operation dar (Jeha et al. 2007).

\subsubsection{Therapie}

Die Therapie richtet sich nach der vorliegenden Epilepsieform und ist daher sehr unterschiedlich (vergleiche Deutsche Gesellschaft für Neurologie 2013: S1-Leitlinie „Erster epileptischer Anfall und Epilepsien im Erwachsenenalter"). Zu den verschiedenen Möglichkeiten zählt die Änderung der Lebensführung (Vermeidung von Provokationsfaktoren, wie zum Beispiel unregelmäßiger Schlafrhythmus), medikamentöse oder chirurgische Therapien sowie neue Verfahren wie zum Beispiel die invasive und die nicht-invasive Hirnstimulation.

Bei der konservativen Behandlung wird zunächst eine Monotherapie versucht. Die Wahl des entsprechenden Medikaments ist dabei von der jeweiligen Epilepsieform abhängig. Zeigen sich eine Therapieresistenz oder starke Nebenwirkungen, wie zum Beispiel eine massive Gewichtszunahme oder die Gefahr fetaler Missbildungen bei Gravidität, wird ein Alternativpräparat verabreicht. Auch Kombinationen verschiedener Antiepileptika stellen eine Möglichkeit dar, wenn kein zufriendenstellender Therapieerfolg eintritt. Bei ca. $30 \%$ der Patienten kann jedoch trotz Mehrfachkombination und Aufdosierung von Medikamenten keine zufriedenstellende Anfallshäufigkeit eingestellt werden (Kwan und Brodie 2000).

Die chirurgische Therapie bietet insbesondere bei pharmakoresistenten fokalen Epilepsien eine gute Alternative (Mikati et al. 2006; Rosenow und Lüders 2001). Dazu muss das epileptogene Areal genau detektierbar und die operative Entfernung dieses Bereichs hinreichend und notwendig für eine deutliche Verbesserung des Anfallsgeschehens sein (Rosenow und Lüders 2001). Die häufigsten chirurgischen Eingriffe stellen hierbei die Resektion des vorderen Temporallappens, des epileptogenen Areals im Frontallappen und die Amygdala-Hippokampektomie dar (Hacke 2010; Jeha et al. 2007).

In entsprechenden Zentren lässt sich bei Patienten mit Temporallappenepilepsie durch die Temporallappenresektion in ca. $50 \%$ der Fälle eine Anfallsfreiheit feststellen und bei weiteren $25 \%$ stellt sich eine deutliche Anfallsreduktion ein (Hacke 2010; Jeha et al 2007). Neben der konservativen und der chirurgischen Therapie rücken weitere Verfahren in den wissenschaftlichen Fokus. Zu diesen zählen die invasive, tiefe Hirnstimulation (DBS) sowie nebenwirkungsärmere, nicht-invasive Stimulationstechniken, wie z.B. die repetitive transkranielle Magnetstimulation (rTMS) oder die transkranielle Gleichstromstimulation (tDCS). Diese beeinflussen durch magnetische bzw. elektrische Impulse die Erregbarkeit kortikaler Neurone und könnten so die Entstehung epileptischer Anfälle beeinflussen. Die 
Effektivität dieser Verfahren in der Behandlung von Epilepsien ist jedoch schwierig zu validieren, da Studien in diesem Gebiet sehr heterogen bzgl. des Studiendesigns, der Epilepsieformen und der Größe der Patientenkollektive erscheinen (Lefaucheur et al. 2014, Alexander 2013). Vor allem bei Untersuchungen mit Epilepsie-Patienten mit neokortikaler Epilepsie oder mit fokal-kortikalen Dysplasien zeigte sich jedoch ein gutes Ansprechen auf die rTMS im Sinne einer erfolgreichen Anfallsreduktion (Hsu et al. 2011).

Bezüglich der DBS konnte in verschiedenen Studien (Fisher et al. 2010; Osorio et al. 2007; Kerrigan et al. 2004) gezeigt werden, dass die elektrische Stimulation tiefer Hirnregionen, insbesondere des anterioren Thalamuskerns, ebenfalls zu einer Reduktion der Anfallsfrequenz sowie der Anfallsschwere führen kann.

Die Wirksamkeit der Tiefenstimulation bestimmter Hirnregionen konnte bereits bei anderen neurologischen Erkrankungen, wie zum Beispiel bei dem idiopathischen Parkinson-Syndrom, aufgezeigt werden und stellt dort ein etabliertes Verfahren dar (Wojtecki et al. 2007). Bei therapierefraktären Epilepsie-Patienten wird nun versucht durch elektrische Reize verschiedener Frequenzen die Übererregbarkeit zu reduzieren. Dies geschieht über eine Inaktivierung exzitatorischer sowie über die Aktivierung inhibitorischer Neuronen und der Modulation neuronaler Netzwerke (Schulze-Bonhage 2009). Die Beobachtungen in einer großen multizentrischen Studie in den USA (SANTE) ergaben dabei einen durchschnittlichen Rückgang der Anfallsfrequenzen um 56 \% zwei Jahre nach der Implantation. Bei $54 \%$ der Patienten konnte eine Reduktion der Anfälle um mindestens $50 \%$ festgestellt werden und 14 von 110 Patienten waren vollständig anfallsfrei (Fisher et al. 2010).

\subsection{Bedeutung der MRT-Diagnostik in der Epileptologie}

Die Magnetresonanztomographie ist das präferierte bildgebende Verfahren in der Diagnostik von Epilepsie und dem CT in Sensitivität und Spezifität überlegen (ILAE Commission Report 1997). Es besitzt einen höheren Weichteilkontrast, eine bessere Raumauflösung, die Möglichkeit einer multiplanaren Darstellung und im Gegensatz zum CT wird keine ionisierende Strahlung verwendet (Chinchure et al. 2010). Darüber hinaus ist es obligat bezüglich der Lokalisation epileptogener Foki und einer hochauflösenden strukturellen Bildgebung, die insbesondere in der prächirurgischen Diagnostik einen hohen Stellenwert besitzt, da sich die Kenntnis eines epileptogenen Areals als wegweisend für den Erfolg einer Operation erwiesen hat (Lai et al. 2010; Jeha et al 2007). Das strukturelle MRT stellt damit ein wichtiges bildgebendes Verfahren dar, da sich ein chirurgischer Eingriff bei pharmakoresistenten Patienten mit fokaler Epilepsie als wertvolle Alternative bezüglich der Anfallssituation und der Lebensqualität herausgestellt hat (Mikati et al. 2006; Rosenow und Lüders 2001; Wiebe et al. 2001). Bei 30 \% aller Patienten mit fokaler Epilepsie ist das 
Routine-MRT hingegen unauffällig, wodurch diese deutlich niedrigere Erfolgschancen bei einer möglichen Entfernung des epileptogenen Areals besitzen (Pastor et al. 2010; Jeha et al. 2007; Berkovic et al. 1995). Eine Erhöhung der Detektionsrate vermuteter Läsionen bei diesen Patienten erscheint daher wünschenswert.

\section{$1.4 \quad$ Zielsetzung dieser Studie}

Das Ziel dieser Studie ist es, eine Verbesserung des Verständnisses von idiopathischgeneralisierten Epilepsien unter besonderer Berücksichtigung des Janz-Syndroms zu erarbeiten und damit den Weg für eine Verbesserung diagnostischer Maßnahmen zu ebnen.

Wie oben beschrieben, handelt es sich bei den idiopathisch-generalisierten Epilepsien um eine definierte Gruppe, bei der von einer genetischen Disposition ausgegangen wird, ohne dass sich bei den Patienten strukturelle Alterationen im Routine-MRT finden lassen. Die Einteilung erfolgt dabei basierend auf klinischen Charakteristika sowie typischen Veränderungen im EEG. Bisherige Studien konnten mittels VBM dezente Veränderungen in der grauen bzw. weißen Substanz insbesondere bei Patienten mit JME nachweisen, wobei die Ergebnisse kein einheitliches Bild abgeben oder widersprüchlich erscheinen (O'Muircheartaigh et al. 2011; Betting et al. 2006; Woermann et al. 1999). Zu ähnlich uneinheitlichen Ergebnissen gelangen andere Studien, bei denen eine weitere IGEUntergruppe, IGE mit isolierten tonisch-klonischen Anfällen, untersucht wurde (Huang et al. 2011; Bernhardt et al. 2009; Betting et al. 2006).

DTI-Studien bei JME-Patienten weisen hingegen relativ einheitlich auf Veränderungen innerhalb zerebraler Netzwerke hin und werfen damit die Frage auf, inwiefern Veränderungen der weißen Substanz eine bedeutendere Rolle bei JME/IGE spielen könnten (Liu et al. 2011; Vulliemoz et al. 2011).

In dieser Arbeit sollen daher mögliche strukturelle Alterationen der weißen bzw. der grauen Substanz mittels VBM untersucht sowie Störungen zerebraler Netzwerke mittels einer DTIAnalyse detektiert werden. Im Interessenfokus stehen dabei weitere Erkenntnisse bezüglich der Betroffenheit dieser Strukturen und Netzwerke, um eventuell Wege für neue Therapie-/ Diagnosemöglichkeiten zu bahnen. Des Weiteren sollen Erkenntnisse über den Nutzen verschiedener MRT-Verfahren (T1-gewichtete Sequenz, MT-Karte, T1-Relaxometrie, DTI) erforscht werden. Dabei werden eine klassische T1-gewichtete Sequenz sowie neuartige quantitative Karten (MT-Karte, T1-Relaxometrie) verwendet, um mögliche Unterschiede in der Detektion struktureller Veränderungen aufzuzeigen. 


\section{Material und Methoden}

Es handelt sich bei dieser Arbeit um eine prospektive, klinische Studie mit Kontrollgruppe.

Sie entstand im Rahmen der Studie „Multi-Parameter Mapping bei fokaler und generalisierter Epilepsie“ unter der Projektleitung von Prof. Dr. med. Paulus, Prof. Dr. med. Knauth und von PD Dr. rer. nat. Dechent an der Universitätsmedizin Göttingen (UMG). Prüfarzt war Dr. med. Focke. Sie wurde von der Ethikkommission der Georg-August-Universität zu Göttingen genehmigt (03/06/08).

\section{$2.1 \quad$ MRT-Bildgebung}

Die Messung der Patienten sowie der Kontrollprobanden wurde im MR-Tomographen der Abteilung MR-Forschung der Neurologie und Psychiatrie der Universitätsmedizin Göttingen durchgeführt. Die Untersuchung fand einmalig im MRT (Magnetom Trio, Siemens Healthcare, Erlangen, Deutschland) mit einer Feldstärke von drei Tesla unter Verwendung einer 8-Kanal-Kopfspule (Invivo Corporation, Gainesville, Florida, Vereinigte Staaten von Amerika) statt. Eine Kontrastmittelgabe wurde nicht vorgenommen.

Die MR-Untersuchung wurde von den Medizinisch-Technischen Radiologie-Assistentinnen (MTRA) der UMG übernommen. Diese betreuten die Patienten sowie die Kontrollprobanden während der gesamten Messung und standen mit ihnen durchgehend über eine Gegensprechanlage in Kontakt. Die zu untersuchenden Personen befanden sich dabei in Rückenlage auf dem MRT-Tisch. Der Kopfbereich wurde durch seitliche Schaumstoffkeile gepolstert um mögliche Artefakte durch Kopfbewegungen zu vermindern. Zudem erhielten die Kontrollprobanden und Patienten einen Gehörschutz sowie einen Notfallknopf, mit dem die Messung jederzeit abgebrochen werden konnte.

Die zerebrale Bildgebung erfolgte anhand der Sequenzen des folgenden Protokolls der Studie „Multi-Parameter Mapping bei fokaler und generalisierter Epilepsie“ (siehe oben): 


\begin{tabular}{|l|l|}
\hline 1 & MP-RAGE 3D Volume Scan (1mm isotrop) \\
\hline 2 & FLAIR 3mm coronar \\
\hline 3 & MT-Mapping (3D-T1w) \\
\hline 4 & MT-Mapping (3D-PDw) \\
\hline 5 & MT-Mapping (3D-MTw) \\
\hline 6 & T2-Mapping \\
\hline 7 & EPI-DTI \\
\hline
\end{tabular}

Tabelle 1: Übersicht MRT-Messung

Für diese Promotionsarbeit wurden die MP-RAGE 3D-, die DTI-Sequenz sowie die MTKarten verwendet. Die Speicherung der Daten erfolgte zunächst im DICOM-Format. Anschließend wurden diese auf einen Bilderserver (Linux Betriebssystem) übertragen, die Konvertierung zu 3D NIFTI-Dateien mit der Software mriconvert (http://lcni.uoregon.edu/ jolinda/MRIConvert) vorgenommen und die Qualität der Bilder beurteilt (major/minor problems, good). Zudem wurden die Datensätze pseudonymisiert und unter den Pseudonymen ebenfalls weitere relevante Informationen (zum Beispiel Alter, Geschlecht, usw.) eingegeben wurden.

\subsubsection{T1-gewichtete Bildgebung}

Die T1-gewichtete Bildgebung gehört zu den Standardsequenzen im Routine-MRT bei Epilepsie-Patienten (Deutsche Gesellschaft für Neurologie 2013: S1-Leitlinie „Erster epileptischer Anfall und Epilepsien im Erwachsenenalter"; Wehner und Lüders 2008).

Sie bietet eine gute Möglichkeit der Darstellung anatomischer, insbesondere zerebraler Strukturen. Fettreiche Areale (zum Beispiel weiße Substanz) erscheinen dabei aufgrund ihrer kurzen T1-Relaxationszeit hyperintens, weniger fettreiche Strukturen (zum Bespiel graue Substanz) oder Flüssigkeiten sind im T1-gewichteten MR-Bild hypointens (Reiser et al. 2011, vergleiche Kapitel 1.1.2). Somit stellt sich eine gute Kontrastierung zwischen diesen Geweben dar, was in dieser Studie genutzt wurde.

Zu Beginn der MR-Messung wurde eine T1-gewichtete Gradientenecho-Sequenz mit 1mm isotroper Auflösung und Magnetisierungsvorbereitung (3D-MP RAGE Volume Scan, vergleiche Tabelle 1) durchgeführt. Es wurde eine TR von $2250 \mathrm{~ms}$ und eine TE von 3,0 3,2 ms gewählt. Der Anregungswinkel betrug $9^{\circ}$, die Inversionszeit $900 \mathrm{~ms}$, die Auflösung $1,0 \times 1,0 \times 1,0 \mathrm{~mm}$ und die Matrix $176 \times 256 \times 232$. 


\subsubsection{Multi-Parameter-Mapping}

In dieser Arbeit wurden neben der Diffusions-Tensor- sowie der oben genannten T1gewichteten Bildgebung auch zwei quantitative Verfahren verwendet. Die Magnetisierungstransfer-Karte (MT-Karte) sowie die T1-Relaxometrie basieren dabei auf Berechnungen von Daten aus den MT-Mapping-Sequenzen (siehe Tabelle 1), wobei der T1Relaxometrie die Daten der PD- sowie der T1-gewichteten Sequenzen und der MT-Karte zusätzlich noch die Werte aus der MT-gewichteten Messung zugrunde liegen.

Die Magnetisierungs-Transfer-Bildgebung beruht auf dem sogenannten Magnetisierungstransferkontrast (magnetization transfer contrast, MTC, vergleiche Wolff und Balaban 1989). Um Defekte von immobilen Molekularstrukturen besser darstellen zu können, zum Beispiel von fettreichen Geweben wie Myelinscheiden, wird bei dieser Technik die unterschiedlich weite Bandbreite der Lamorfrequenzen von freien und makromolekularen Wasserstoffkernen genutzt. Letztere besitzen hier ein deutlich breiteres Spektrum und können somit durch einen entsprechenden HF-Impuls separat angeregt werden (Weishaupt et al. 2009). Da sich makromolekulare und in freiem Wasser vorliegende Protonen durch Spin-SpinWechselwirkungen bzw. durch chemische Vorgänge gegenseitig beeinflussen, findet zwischen ihnen eine Übertragung der Magnetisierung statt (Weishaupt et al. 2009; Reiser und Semmler 2002). Durch den Magnetisierungs-Transfer (MT) der magnetisch gesättigten makromolekularen Protonen auf die freien Wasserstoffkerne, sinkt dort die Signalintensität (Reiser und Semmler 2002). Dieses Magnetisierungtransfersverhältnis (MTR) wird dabei in der Bildgebung verwendet, da es einen Indikator für die Integrität verschiedener Strukturen, wie zum Beispiel Myelinscheiden, darstellt (Cercignani 2001; Filippi et al. 1999).

Das MTR der verschiedenen Gewebe ist jedoch keine feste Größe, sondern wird durch verschiedene Einflüsse, wie zum Beispiel Sequenzparameter oder T1-Relaxation, verändert (Helms et al. 2008). Um die Sättigung durch einen MT-Impuls während der TR besser abschätzen zu können, wurde in der MR-Forschung in der Neurologie und Psychiatrie der UMG ein semiquantitativer Parameter erarbeitet (vergleiche Helms et al. 2008). Hierzu werden, basierend auf einer 3D-FLASH-Sequenz, zwei Referenzmessungen (T1- und PDgewichtet) verwendet und der berechnete Einfluss eines MT-Pulses als empirischer Term in die MT-Karte mit einbezogen. Diese MT-Karte besitzt eine gute Kontrastierung und eignet sich insbesondere zur Darstellung lipider Strukturen, wie zum Beispiel der weißen Substanz. Die T1-Relaxometrie als zweites hier verwendetes quantitatives Verfahren erlaubt eine bessere Abschätzung der tatsächlichen T1-Relaxation. Die in einer T1-gewichteten Sequenz gemessene Signalintensität wird neben der T1-Relaxtion auch von weiteren Parametern, 
insbesondere von der Protonendichte in der FLASH-Sequenz, beeinflusst (Deoni 2010). Dieser Einfluss wird in der T1-Relaxometrie anhand einer T1- sowie einer PD-gewichteten Referenzmessung kalkuliert und bei der Berechnung der quantitativen Karte (R1-Karte) berücksichtigt (Gringel et al. 2009; Helms et al. 2008).

Bei der T1- sowie bei der PD-basierten Messung wurde eine TR von 23 ms sowie ein Flipwinkel von $20^{\circ}(\mathrm{T} 1)$ bzw. $6^{\circ}$ (PD) benutzt. Bei der MT-gewichteten Sequenz wurde eine TR von 38 ms sowie ein Flipwinkel von $9^{\circ}$ eingesetzt. Die Echozeiten betrugen 2,46, 4,92, 7,38, 9,84, 12,3, 14,76, 17,22 und 19,68 ms. Es wurde eine Auflösung von 1,0 x 1,0 x 1,0 mm sowie eine Matrix von $160 \times 256$ × 232 gewählt.

\subsubsection{Diffusions-Tensor-gewichtete Bildgebung (DTI)}

Die DTI ist eine weiterentwickelte Form der Diffusionsgewichteten Bildgebung (diffusion weighted imaging, $D W I$ ) und ermöglicht die räumliche Darstellung von Nervenfasern, basierend auf den Diffusionsbewegungen von Wassermolekülen. Diese beruhen auf dem Prinzip der Brownschen Molekularbewegung. Die Wassermoleküle verteilen sich in Flüssigkeiten, zum Beispiel im Liquor cerebrospinalis, weitestgehend zufällig. Diese stochastisch erfolgende Diffusion wird als „isotrop“ bezeichnet. In bestimmten Geweben wird die Diffusion, zum Bespiel durch Zellmembranen begrenzt und erfolgt daher "gerichtet“ oder „anisotrop“. Diese Diffusionsbewegung entlang der parallelen Nervenfasern geschieht dabei schneller als in andere Richtungen. Neben dem mittleren Diffusionskoeffizienten (MD) kann das Diffusionsverhalten für eine bestimmte Volumeneinheit anhand der Gerichtetheit der Diffusionsbewegungen mit Hilfe der fraktionellen Anisotropie (FA) aufgezeigt werden. Es gelten hier Werte von 0 bis 1 , wobei 1 für eine völlig gleichgerichtete Anisotropie und 0 für eine komplette Ungerichtetheit der Molekülbewegungen steht. Je höher die Anisotropie einer Volumeneinheit ist, desto mehr gleichgerichtete Nervenfasern werden dort vermutet (Johansen-Berg und Behrens 2009; Weishaupt et al. 2009; Le Bihan et al. 2001).

Bei der im Protokoll verwendeten DTI-Messung wurde eine Echo-Planar-Imaging (EPI)-2DSequenz benutzt. Neben einer Referenzmessung ohne Diffusionswichtung (B0) wurden dabei jeweils 30 diffusionsgewichtete Bildvolumen (30 nicht kollinearen Diffusionsrichtungen, B1, b-Wert $=1000 \mathrm{~s} / \mathrm{mm}^{2}$ ) erhoben. Die TR betrug $10.000 \mathrm{~ms}$, die TE $93 \mathrm{~ms}$ und der Flipwinkel $90^{\circ}$. Es wurde eine Auflösung von $1,9 \times 1,9 \times 1,9 \mathrm{~mm}$ und eine Matrix von $128 \mathrm{x}$ 74 x 128 gewählt. 


\subsection{Patientenkollektiv}

In dieser Studie wurden Patienten der Universitätsmedizin Göttingen untersucht, die die Einschlusskriterien erfüllten und bei denen keine Ausschlusskriterien vorlagen. Sie wurden vor der Studienteilnahme ausführlich über die Untersuchung informiert und erklärten schriftlich ihr Einverständnis an der Studienteilnahme (siehe Anhang).

\subsubsection{Einschlusskriterien}

Alle Patienten sollten zum Untersuchungszeitpunkt folgende Kriterien erfüllen:

- Klinisch-elektrophysiologisch gesicherte Diagnose (IGE, insbesondere juvenile myoklonische Epilepsie)

- Keine weiteren relevanten neurologischen Erkrankungen

- Keine stattgefundene ZNS-Operation

- Keine bekannten zerebralen Auffälligkeiten durch vorherige bildgebende Verfahren

Als Kontrollprobanden wurden gesunde Personen untersucht, bei denen zum Zeitpunkt der Studienteilnahme keine relevanten neurologischen Erkrankungen bzw. Ausschlusskriterien für eine MRT-Untersuchung vorlagen. Die Kontrollgruppe sollte eine ähnliche Altersstruktur und Geschlechterverteilung wie die Patientengruppen aufweisen (siehe Kapitel 2.3).

\subsubsection{Ausschlusskriterien}

Es galten die Ausschlusskriterien der oben beschriebenen Studie „Multi-Parameter Mapping bei fokaler und generalisierter Epilepsie“. Neben ethischen und rechtlichen Gesichtspunkten (siehe unten) stand die Tauglichkeit für eine MRT-Untersuchung im Vordergrund. Wichtige Kontraindikationen wurden daher frühzeitig abgefragt. Zudem musste jeder Patient/Proband vor der Messung einen Fragebogen ausfüllen, woraufhin die Eignung für eine Untersuchung im 3-Tesla-MRT endgültig geprüft wurde (siehe Anhang). Personen, bei denen eine MRTUntersuchung nicht durchführbar war, wurden, soweit bekannt, im Vorfeld aus der Studie ausgeschlossen. Hierzu zählten beispielsweise körperliche Aspekte wie starkes Übergewicht (> $130 \mathrm{~kg}$ ) sowie ausgeprägte Erkrankungen des Bewegungsapparats, die eine Untersuchung im MRT aufgrund unzureichender Lagerungsmöglichkeiten nicht erlaubten. Ebenfalls konnten Patienten oder Kontrollprobanden nicht in die Studie aufgenommen werden, bei denen aufgrund von intrakorporalen metallischen Materialien starke und nicht zu korrigierende Bildartefakte zu erwarten waren. 
Zudem wurden Patienten oder Kontrollprobanden, die eines der folgenden Kriterien erfüllten, aus der Studie ausgeschlossen:

- Alter $<18$ oder $>60$ Jahre

- Reduzierter Bewusstseinszustand

- Eingeschränkte Einsichtsfähigkeit

- Gravidität (bestehend oder vermutet)

- Substanzabhängigkeit (Drogen/ Medikamente/ Alkohol)

- Herzschrittmacher

- Metallimplantate, die nicht für eine Untersuchung in einem 3-Tesla-MRT geeignet sind

- Andere im Körper befindliche Metallteile, die zu einer Beeinträchtigung des Patienten führen könnten

- Klaustrophobie

\subsection{Datenerhebung}

Neben den MRT-Messungen sowie den allgemeinen Informationen (Name, Alter, Geschlecht) wurden folgende klinische Parameter erhoben und anonymisiert gespeichert:

- Form der Epilepsie

- Alter zu Erkrankungsbeginn

- Vorgeschichte (Geburtskomplikationen, Fieberkrämpfe, SH-Traumata, Infektionen)

- Ergebnisse der EEG-Untersuchung

- Ergebnisse sonstiger radiologischer Untersuchungen

- Anfallsfreiheit bzw. Anfallshäufigkeit pro Jahr

Es wurden insgesamt 13 Patienten mit idiopathisch-generalisierter Epilepsie (ohne JME, Gruppe IGE), 12 Patienten mit juveniler myoklonischer Epilepsie (Gruppe JME) und 44 gesunde Kontrollprobanden (Gruppe Cx) im MRT der MR-Forschung der Neurologie und Psychiatrie der UMG untersucht. Für die TBSS-basierte Analyse konnten die Daten von allen oben genannten Patienten und Probanden berücksichtigt werden. Bei der VBM-basierten Untersuchung konnten aufgrund von qualitativen Problemen (zum Beispiel verminderte Bildqualität durch Bewegungen des Probanden/Patienten während der entsprechenden Sequenzen) nur die Daten von 11 JME-Patienten und 38 Kontrollprobanden mit einbezogen werden. 
Die Kontrollgruppe für die VBM-Untersuchung bestand aus 17 Männern und 21 Frauen (Altersdurchschnitt 31,8 ( \pm 8,9) Jahre, Median 29 Jahre). Bei der TBSS-Untersuchung waren 23 Frauen und 21 Männer in der Kontrollgruppe. Ihr Altersdurchschnitt lag bei 31,9 $( \pm 8,2)$ Jahren und der Median bei 30 Jahren.

Die Gruppe IGE bestand aus 6 Frauen und 7 Männern. Ihr Altersdurchschnitt lag bei 30,2 ( \pm 11,2) Jahren und der Median bei 25 Jahren (vergleiche Tabelle 2).

\begin{tabular}{|c|c|c|c|c|c|}
\hline Pat-Nr. & $\begin{array}{c}\text { Alter } \\
\text { (Jahre) }\end{array}$ & Geschlecht & $\begin{array}{c}\text { Dauer der } \\
\text { Epilepsie } \\
\text { (Jahre) }\end{array}$ & $\begin{array}{c}\text { Beginn der } \\
\text { Epilepsie } \\
\text { (Alter in Jahren) }\end{array}$ & anfallsfrei \\
\hline 1 & 32 & männlich & 24 & 8 & nein \\
\hline 2 & 46 & männlich & 33 & 13 & ja \\
\hline 3 & 48 & weiblich & 41 & 7 & ja \\
\hline 4 & 48 & männlich & 24 & 24 & nein \\
\hline 5 & 31 & weiblich & 21 & 10 & ja \\
\hline 6 & 25 & männlich & 6 & 19 & ja \\
\hline 7 & 19 & männlich & 3 & 16 & ja \\
\hline 8 & 25 & weiblich & 10 & 15 & jein \\
\hline 9 & 22 & weiblich & 5 & 17 & nein \\
\hline 10 & 18 & männlich & 2 & 15 & nein \\
\hline 11 & 37 & männlich & 22 & 12 & ja \\
\hline 12 & 23 & weiblich & 11 & 13 & ja \\
\hline 13 & 19 & weiblich & 6 & & \\
\hline
\end{tabular}

Tabelle 2: Patienten mit idiopathisch-generalisierter Epilepsie (ohne JME)

Die Gruppe JME (VBM) bestand aus 6 Frauen und 5 Männern. Ihr Altersdurchschnitt betrug 33,1 $( \pm 7,8)$ und der Median 35 Jahre. In der TBSS-Analyse bestand die Gruppe JME aus 6 Frauen und 6 Männern. Ihr Altersdurchschnitt lag bei 33,1 ( $\pm 7,5)$ und der Median bei 34 Jahren (vergleiche Tabelle 3). 


\begin{tabular}{|c|c|c|c|c|c|}
\hline Pat.-Nr. & $\begin{array}{c}\text { Alter } \\
\text { (Jahre) }\end{array}$ & Geschlecht & $\begin{array}{c}\text { Dauer der } \\
\text { Epilepsie } \\
\text { (Jahre) }\end{array}$ & $\begin{array}{c}\text { Beginn der } \\
\text { Epilepsie } \\
\text { (Alter in Jahren) }\end{array}$ & anfallsfrei \\
\hline 1 & 28 & weiblich & 11 & 17 & nein \\
\hline 2 & 40 & weiblich & 24 & 16 & ja \\
\hline 3 & 24 & weiblich & 12 & 12 & nein \\
\hline 4 & 36 & männlich & 10 & 26 & ja \\
\hline 5 & 21 & männlich & 3 & 18 & nein \\
\hline 6 & 46 & männlich & 39 & 7 & nein \\
\hline 7 & 25 & männlich & 6 & 19 & nein \\
\hline 8 & 33 & weiblich & 15 & 18 & ja \\
\hline 9 & 35 & weiblich & 22 & 13 & nein \\
\hline 10 & 41 & weiblich & 28 & 13 & ja \\
\hline 11 & 35 & männlich & 20 & 15 & nein \\
\hline 12 & 33 & männlich & 2 & 31 & nein \\
\hline
\end{tabular}

Tabelle 3: Patienten mit juveniler myoklonischer Epilepsie (JME)

Anhand Voxel-basierter Morphometrie (VBM) und Tract-based Spatial Statistics (TBSS) wurden vier Vergleiche unter den Gruppen durchgeführt. Dabei wurden die gemeinsame Patientengruppe (IGE und JME) sowie beide Patientengruppen einzeln der Kontrollgruppe gegenübergestellt. Zudem wurden beide Patientengruppen direkt miteinander verglichen.

\subsection{Analyse und Auswertung}

Die Auswertung der T1-Sequenzen sowie der MT-Karten erfolgte mittels Voxel-basierter Morphometrie, die DTI-Sequenzen wurden mittels TBSS analysiert.

\subsubsection{Voxel-basierte Morphometrie (VBM)}

Die VBM wurde mit Hilfe der Statistical Parametric Mapping Version 2008 (SPM8, http://www.fil.ion.ucl.ac.uk/spm) und dem Programm Matlab 7.7 (The MathWorks Inc., Natick, Massachusetts, Vereinigte Staaten von Amerika) durchgeführt.

Dieses Verfahren ermöglicht einen Voxel-basierten Vergleich von lokalen Volumina zerebraler Strukturen, zum Beispiel von der grauen Substanz, zwischen zwei Gruppen (vergleiche Hobbs und Novak 2008; Salmond et al. 2002; Ashburner und Friston 2000; Ashburner und Friston 1997). Dabei wird anhand des Intensitätsvergleichs jedes einzelnen Voxels eine statistische parametrische Karte erstellt, mit der signifikante Unterschiede direkt visualisiert werden. Dieses Prinzip beruht auf automatisierten Rechenvorgängen und ist damit deutlich weniger abhängig vom jeweiligen Untersucher. Es müssen weiter keine $a$ priori-Annahmen über die Lokalisation von Veränderungen (region of interest, ROI) vorliegen. 
Zunächst werden die Bilder anhand von Wahrscheinlichkeitskarten in weiße und graue Substanz unterteilt (unified segmentation). Die Intensität jedes einzelnen Voxels wird dabei mit diesen Karten sowie mit Wahrscheinlichkeitswerten (0 - 1) innerhalb desselben Bildes verglichen und so einer der Klassen zugeordnet. Anschließend findet eine Transformation der Daten in einen standardisierten, stereotaktischen Raum (MNI-Raum mit einer Voxelgröße von $1,5 \mathrm{~mm}^{3}$ ) statt, was als räumliche Normalisierung (spatial normalisation) bezeichnet wird. Die Bilder werden dabei durch verschiedene Rechenprozesse in Ausrichtung und Größe einer zuvor kalkulierten, studienspezifischen Maske (template) angepasst, sodass ein späterer rechenbasierter Vergleich weitestgehend unabhängig von Kopfgröße und -form ermöglicht wird. Des Weiteren wird eine Modulation der Daten durchgeführt. Dieser Schritt beinhaltet die Korrektur der durch die zuvor räumliche Normalisierung hervorgerufenen Volumenänderungen. Für die Normalisierung und Modulation wurde dabei die DARTEL Toolbox verwendet (vgl. Ashburner 2007). Abgeschlossen wird die Vorverarbeitung der Daten durch den Prozess des sogenannten Smoothing. Dabei wird jedes Voxel mittels der durchschnittlichen Signalintensität der umgebenden Voxel gewichtet, um eine inakkurate Normierung zu kompensieren. Diese Berechnung beruht auf einem isotropen Gaußkern, wobei die Filtergröße unterschiedlich gewählt werden kann. Sie betrug in dieser Arbeit jeweils $8 \mathrm{~mm}$.

Die abschließende Analyse erfolgte als t-Test für unabhängige Stichproben. Mit einer Fehlerkorrektur für multiple Tests mittels family wise error rate wurde ein $p$-Wert von $p<0,05$ gewählt. Ebenfalls wurde die Analyse ohne strenge Fehlerkorrektur mit einem unkorrigierten P-Wert von $p<0,001$ durchgeführt.

\subsubsection{Tract-Based Spatial Statistics (TBSS)}

Zur Auswertung der DTI-Daten wurde FSL (FMRIB Software Library, Version 4.1, http://www.fmrib.ox.ac.uk/fsl) mit Tract-Based Spatial Statistics (v1.2) verwendet. Diese vom Oxford University Centre for Functional MRI of the Brain (FMRIB) entwickelte Analysemethode erlaubt eine statistische Untersuchung von FA- und MD-Daten verschiedener Patienten bzw. Probanden, wobei diese voxelweise miteinander verglichen werden. Dies ermöglicht einen Rückschluss auf die Konnektivitätsstruktur innerhalb der weißen Substanz basierend auf der anisotropischen Diffusion von Wassermolekülen innerhalb der zerebralen Trakte.

Diese Analysemethode besteht aus der Vorverarbeitung der Daten, verschiedenen Bearbeitungsprozessen sowie der statistischen Auswertung (vergleiche Smith et al. 2007; Basser und Pierpaoli 1996; http://fsl.fmrib.ox.ac.uk/fsl/fslwiki/TBSS/UserGuide). 
Zunächst werden FA- sowie MD-Karten mit dem Programm FDT (FMRIB Software Library, Oxford, FMRIB Analysis Group, „dtifit" tool) berechnet. Dabei werden die DTI-Daten zuvor bezüglich Bewegungs- und Strömungsartefakte mit Hilfe des nicht diffusionsgewichteten B0Referenzbildes korrigiert („eddy-correct“ tool). Im ersten Skript werden verschiedene Berechnungen durchgeführt, um wahrscheinliche Ausreißer innerhalb der diffusionsgewichteten Daten zu detektieren. Zusätzlich werden Übersichtsbilder angefertigt, die die Erkennung weiterer Berechnungsfehler ermöglichen. Der zweite Schritt dient anschließend der nicht-linearen Registrierung der Daten, welche in einem normierten Raum ausgerichtet werden. Im dritten Arbeitsschritt werden nun alle FA- bzw. MD-Daten mit Hilfe der zuvor errechneten, nicht-linearen Transformation in den Standardraum transformiert und ein pseudo-anatomisches „Skelett“ aus den großen Gyri berechnet. Anschließend werden mit Hilfe von distance-maps die FA- und MD-Werte der einzelnen Probanden auf dieses Skelett projiziert und diese Voxel für Voxel miteinander verglichen. Es erfolgte weiter eine Randomisierung mittels „randomise“ (Permutation-based nonparametric inference) und die Cluster wurden unter Optimierung mit TFCE (Threshold-Free Cluster Enhancement) ermittelt (t-Test für unabhängige Stichproben, $p<0,01$, korrigiert für multiple Test). Die resultierenden statistischen Karten wurden mittels MRIcron (http://www.mccauslandcenter.sc.edu/ mricro/mricron/index.html) dargestellt, indem die errechneten FA- bzw. MD-Karten auf die entsprechenden berechneten Skelette projiziert wurden. Voxel mit Werten oberhalb des Schwellenwerts wurden als zusammenhängende Muster (Cluster) unter Verwendung des FSL „cluster"-tools dargestellt. Ebenso erfolgte die Berechnung von Sub-Clustern, die als lokale Maxima einzelner Cluster mit mindestens $8 \mathrm{~mm}$ Abstand definiert wurden (vergleiche Smith et al. 2007). 


\section{$3 \quad$ Ergebnisse}

\subsection{VBM der T1-gewichteten Sequenz und des Multi-Parameter- Mappings}

Die Untersuchung der gewonnenen Daten aus der T1-gewichteten Sequenz sowie aus der MT-Karte und der T1-Relaxometrie zeigte sowohl Substanzzunahmen als auch -abnahmen in der grauen bzw. weißen Substanz bei beiden Patientengruppen im Vergleich zu der Kontrollgruppe mit einer Wahrscheinlichkeit von $p<0,001$ (t-Test für unabhängige Stichproben, unkorrigiert bezüglich des peak level- sowie des spatial extent family wise errors). Unter Einbeziehung der Korrektur für multiple Tests erreichte kein Wert das Signifikanzniveau von $\mathrm{p}<0,05$. Die Zuordnung der Cluster zu zerebralen Strukturen erfolgte anhand des FSL-Atlas "Harvard-Oxford cortical and subcortical structural atlas". Aufgelistet wurden dabei alle Cluster mit einer Voxelgröße von mehr als zehn Voxel.

Erläuterungen zu den folgenden Tabellen:

$\mathrm{p}(\mathrm{FWE})=\mathrm{FWE}$-korrigierter $\mathrm{p}$-Wert bezüglich der Voxelintensität (peak level), $\mathrm{p}(\mathrm{FWE} /$ cluster) $=$ FWE-korrigierter $\mathrm{p}$-Wert bezüglich der Clustergröße (spatial extent), $\mathrm{k}=$ Clustergröße in Voxel, $\mathrm{T}=\mathrm{T}$-Wert, $\mathrm{MNI}=$ Koordinaten auf der $\mathrm{x}$-, y- sowie z-Achse (orientierend am Standardgehirn des Montreal Neurological Instituts). 


\subsubsection{Vergleich beider Patientengruppen mit der Kontrollgruppe}

Die VBM der Daten aus der T1-gewichteten Sequenz sowie aus der MT-Karte und aus der T1-Relaxometrie zeigte einen Verlust der grauen Substanz $(p<0,001$, unkorrigiert, vergleiche Abbildung 1) bei der Patientengruppe (IGE mit JME) im Vergleich zur Kontrollgruppe in der supplementär-motorischen Rinde des Motorkortex (hrT1, calcMT.MT, calcMT.R1: 143 46) und im rechten frontoorbitalen Kortex (hrT1: 2432 -14, calcMT.MT: 21 32 -14, calcMT.R1: 2232 -11). Im Bereich des linken frontoorbitalen Kortex (calcMT.MT: -21 29 -14, calcMT.R1: -21 27 -12) fand sich in der MT-Karte sowie in der T1-Relaxometrie ebenfalls eine Verminderung der grauen Substanz. Weitere kleine Cluster wurden in den Daten der T1-gewichteten Sequenz (rechter postzentraler Gyrus: 47 -18 45, linker postzentraler Gyrus: -8 -33 61) sowie in den Daten aus der MT-Karte (linker präzentraler Gyrus: -4 -22 55) detektiert. Es stellten sich ebenfalls kleine Cluster im rechten Gyrus frontalis inferior (hrT1: 4718 22) sowie im lateralen okzipitalen Kortex rechts (calcMT.MT: 24 -70 34) dar.

\begin{tabular}{|c|c|c|c|c|c|c|c|}
\hline & $p$ (FWE) & $\mathrm{p}$ (FWE/cluster) & $\mathbf{k}$ & $\mathbf{T}$ & \multicolumn{3}{|c|}{ MNI* } \\
\hline \multirow[t]{5}{*}{ hrT1 } & 0,858 & 0,985 & 27 & 3,79 & 14 & 3 & 46 \\
\hline & 0,930 & 0,980 & 33 & 3,67 & -8 & -33 & 61 \\
\hline & 0,981 & 0,911 & 79 & 3,50 & 24 & 32 & -14 \\
\hline & 0,985 & 0,994 & 14 & 3,48 & 47 & 18 & 22 \\
\hline & 0,993 & 0,996 & 10 & 3,41 & 47 & -18 & 45 \\
\hline \multirow[t]{6}{*}{ calcMT.MT } & 0,814 & 0,704 & 160 & 3,87 & 21 & 32 & -14 \\
\hline & 0,829 & 0,980 & 35 & 3,85 & 14 & 3 & 46 \\
\hline & 0,971 & 0,891 & 88 & 3,57 & -21 & 29 & -14 \\
\hline & 0,997 & & & 3,35 & -30 & 36 & -15 \\
\hline & 0,992 & 0,992 & 20 & 3,44 & -4 & -22 & 55 \\
\hline & 0,993 & 0,995 & 13 & 3,42 & 24 & -70 & 34 \\
\hline \multirow[t]{3}{*}{ calcMT.R1 } & 0,362 & 0,413 & 285 & 4,36 & 22 & 32 & -11 \\
\hline & 0,822 & 0,981 & 35 & 3,86 & 14 & 3 & 46 \\
\hline & 0,951 & 0,935 & 68 & 3,64 & -21 & 27 & -12 \\
\hline
\end{tabular}

Tabelle 4: IGE + JME < Cx (graue Substanz)

Innerhalb der weißen Substanz zeigte sich im Bereich des rechten Thalamus (hrT1: 14 -30 6, 20 -25 -6, calcMT.MT: $14-30$ 6, 16 -22 -2) eine Volumenabnahme im Vergleich zu den Werten der Kontrollgruppe ( $p<0,001$, unkorrigiert, vergleiche Abbildung 1). Ebenso fand sich in der Voxel-basierten Morphometrie aus den Daten aller drei Sequenzen/Karten ein Verlust des Volumens innerhalb der weißen Substanz im linken Gyrus frontalis superior 
(hrT1, calcMT.MT: -6 18 52, calcMT.R1: -4 18 52), im Bereich des Hirnstamms (hrT1: 3 -12 -21, -6 -10 -23, calcMT.MT: 0 -13 -18, calcMT.R1: $6-10-21,-6-10-21)$ und des linken Gyrus postcentralis (hrT1: -18 -37 51, calcMT.MT: -9 -40 67, -12 -37 52, calcMT.R1: -20 -36 51).

Die Untersuchung der T1-gewichteten Sequenz sowie der MT-Karte konnte auch eine Volumenabnahme der weißen Substanz in der supplementär-motorischen Rinde des Motorkortex links (hrT1, calcMT.MT: $-4-13$ 57), im Bereich des rechten Gyrus cinguli (hrT1: 10 -24 39, 3 -34 25, 30 34, 4 -19 30, calcMT.MT: 3 -34 24, 4 -1 34, 10 -24 39) sowie im linken Gyrus precentralis (hrT1: -15 -24 42, calcMT.MT: -14 -25 40) darstellen. Bei der Analyse der T1-Relaxometrie konnten neben weiteren Arealen (vergleiche Tabelle 5) ein Cluster im Bereich der linken Amygdala (MNI: -14 -6 -12) sowie im Bereich des rechten Nucleus accumbens (MNI: $106-9$ ) detektiert werden. 


\begin{tabular}{|c|c|c|c|c|c|c|c|}
\hline & $p$ (FWE) & $\mathrm{p}$ (FWE/cluster) & k & $\mathbf{T}$ & \multicolumn{3}{|c|}{$\mathrm{MNI}^{*}$} \\
\hline \multirow[t]{14}{*}{ hrT1 } & 0,155 & 0,521 & 181 & 4,51 & -6 & 18 & 52 \\
\hline & 0,243 & 0,064 & 645 & 4,33 & 14 & -30 & 6 \\
\hline & 0,556 & & & 3,94 & 20 & -25 & -6 \\
\hline & 0,387 & 0,366 & 258 & 4,13 & 4 & -3 & -15 \\
\hline & 0,409 & & & 4,10 & -4 & -1 & -14 \\
\hline & 0,406 & 0,543 & 172 & 4,11 & 3 & -12 & -21 \\
\hline & 0,780 & & & 3,70 & -6 & -10 & -23 \\
\hline & 0,667 & 0,849 & 60 & 3,82 & 10 & -24 & 39 \\
\hline & 0,796 & 0,933 & 27 & 3,68 & -4 & -13 & 57 \\
\hline & 0,801 & 0,560 & 165 & 3,67 & -18 & -37 & 51 \\
\hline & 0,873 & 0,933 & 27 & 3,57 & 4 & -19 & 30 \\
\hline & 0,954 & 0,933 & 27 & 3,40 & 3 & -34 & 25 \\
\hline & 0,963 & 0,955 & 17 & 3,37 & -15 & -24 & 42 \\
\hline & 0,969 & 0,944 & 22 & 3,35 & 3 & 0 & 34 \\
\hline \multirow[t]{11}{*}{ calcMT.MT } & 0,340 & 0,450 & 206 & 4,24 & 14 & -30 & 6 \\
\hline & 0,986 & & & 3,32 & 16 & -22 & -2 \\
\hline & 0,421 & 0,721 & 107 & 4,14 & -6 & 18 & 52 \\
\hline & 0,713 & 0,814 & 77 & 3,83 & -14 & -25 & 40 \\
\hline & 0,779 & 0,925 & 37 & 3,75 & 10 & -24 & 39 \\
\hline & 0,813 & 0,943 & 29 & 3,71 & -4 & -13 & 57 \\
\hline & 0,854 & 0,466 & 199 & 3,66 & -9 & -40 & 67 \\
\hline & 0,955 & & & 3,46 & -12 & -37 & 52 \\
\hline & 0,923 & 0,765 & 93 & 3,54 & 0 & -13 & -18 \\
\hline & 0,975 & 0,943 & 29 & 3,38 & 4 & -1 & 34 \\
\hline & 0,983 & 0,974 & 13 & 3,34 & 3 & -34 & 24 \\
\hline \multirow[t]{7}{*}{ calcMT.R1 } & 0,320 & 0,663 & 123 & 4,25 & 6 & -10 & -21 \\
\hline & 0,969 & & & 3,39 & -6 & -10 & -21 \\
\hline & 0,675 & 0,828 & 69 & 3,85 & -4 & 18 & 52 \\
\hline & 0,765 & 0,920 & 36 & 3,75 & 10 & 6 & -9 \\
\hline & 0,795 & 0,406 & 224 & 3,71 & 12 & -51 & -27 \\
\hline & 0,947 & 0,773 & 87 & 3,46 & -14 & -6 & -12 \\
\hline & 0,984 & 0,975 & 10 & 3,31 & -20 & -36 & 51 \\
\hline
\end{tabular}

Tabelle 5: IGE + JME < Cx (weiße Substanz)

Die Analyse der Daten hinsichtlich der Volumenzunahme innerhalb der grauen Substanz der Patientengruppe im Vergleich zu der Kontrollgruppe zeigte eine Zunahme der grauen Substanz ( $p<0,001$, unkorrigiert, vergleiche Abbildung 2 ) im Bereich des linken Gyrus temporalis inferior (hrT1: -48 -54 -21, -46 -39 -15, -50 -31 -20, calcMT.R1: -45 -39 -14). Es wurde weiter in der T1-gewichteten Messung ein kleinerer Bereich im rechten Frontalpol (MNI: 2248 9) detektiert, der ebenfalls in den Daten der quantitativen Bildgebung 
nachgewiesen werden konnte (calcMT.MT: 2250 9, calcMT.R1: 2248 9). Diese zeigten weiter eine Volumenzunahme der grauen Substanz im Gyrus fusiformis links (calcMT.MT: -46 -55 -20, calcMT.R1: $-45-54-20$ ) sowie im linken Frontalpol (calcMT.MT: $-3951-6$, calcMT.R1: -38 51 -6). Weitere Cluster finden sich in Tabelle 6.

\begin{tabular}{|l|c|c|c|c|rrr|}
\hline & $\mathbf{p}(F W E)$ & $\mathbf{p}(F W E /$ cluster) & $\mathbf{k}$ & $\mathbf{T}$ & \multicolumn{3}{|c|}{ MNI* $^{*}$} \\
\hline hrT1 & 0,475 & 0,298 & 361 & 4,21 & -48 & -54 & -21 \\
& 0,804 & & & 3,86 & -46 & -39 & -15 \\
& 0,943 & & & 3,64 & -50 & -31 & -20 \\
& 0,912 & 0,991 & 20 & 3,71 & 22 & 48 & 9 \\
& 0,992 & 0,991 & 19 & 3,42 & 52 & -70 & -15 \\
\hline calcMT.MT & 0,415 & 0,848 & 106 & 4,29 & 47 & 12 & -15 \\
& 0,544 & 0,789 & 129 & 4,16 & -39 & 51 & -6 \\
& 0,858 & 0,987 & 27 & 3,81 & 22 & 50 & 9 \\
& 0,962 & 0,979 & 36 & 3,60 & -46 & -55 & -20 \\
& 0,986 & 0,963 & 49 & 3,49 & 63 & -40 & 16 \\
\hline calcMT.R1 & 0,245 & 0,460 & 262 & 4,53 & -45 & -54 & -20 \\
& 0,946 & & & 3,65 & -51 & -64 & -17 \\
& 0,446 & 0,751 & 146 & 4,26 & -38 & 51 & -6 \\
& 0,963 & 0,994 & 16 & 3,60 & 22 & 48 & 9 \\
& 0,966 & 0,997 & 10 & 3,59 & -39 & -21 & -8 \\
& 0,972 & 0,964 & 50 & 3,57 & 33 & -22 & 1 \\
& 0,983 & 0,987 & 28 & 3,51 & -45 & -39 & -14 \\
\hline
\end{tabular}

Tabelle 6: IGE + JME > Cx (graue Substanz)

Die Untersuchung der weißen Substanz hinsichtlich einer Volumenzunahme bei der Patientengruppe zeigte nur in der quantitativen Bildgebung einen Cluster im Bereich des linken Gyrus fusiformis ( $p<0,001$, unkorrigiert, calcMT.MT: -42 -24 -23, calcMT.R1: -42 -24 -24 , vergleiche Abbildung 2).

\begin{tabular}{|l|c|c|c|c|cc|c|}
\hline & $\mathbf{p}(\mathrm{FWE})$ & $\mathbf{p}(\mathrm{FWE} /$ cluster) & $\mathbf{k}$ & $\mathbf{T}$ & \multicolumn{3}{c|}{ MNI* $^{*}$} \\
\hline hrT1 & \multicolumn{7}{|c|}{ No suprathreshold cluster } \\
\hline calcMT.MT & 0,467 & 0,907 & 44 & 4,09 & -42 & -24 & -23 \\
\hline calcMT.R1 & 0,439 & 0,917 & 37 & 4,10 & -42 & -24 & -24 \\
\hline $\begin{array}{l}\text { * ermittelte Koordinaten mit } \mathrm{p}<0,001, \text { unkorrigiert bezüglich des peak level- sowie des } \\
\text { spatial extent family wise errors }\end{array}$
\end{tabular}

Tabelle 7: IGE + JME > Cx (weiße Substanz) 
A
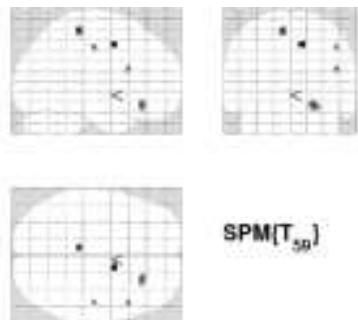

$\left.\operatorname{SPM}_{s p}\right)$

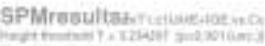

B
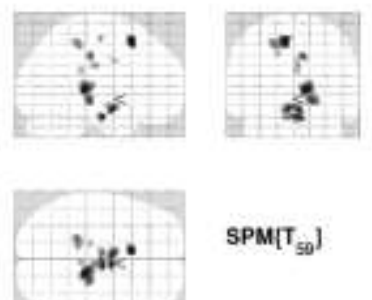

SPM[T $\left.T_{50}\right]$

s.PMresilitst.

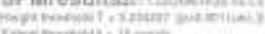

Abbildung 1: JME und IGE < Cx ( A graue Substanz, B weiße Substanz)

A
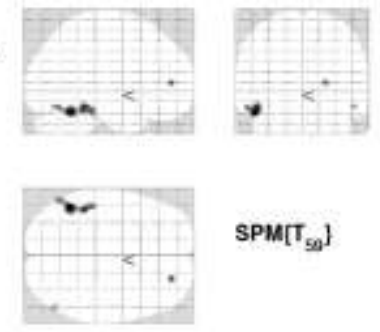

$\operatorname{SPM}\left[T_{S 2}\right]$

5PMrosultstinamainatives

B
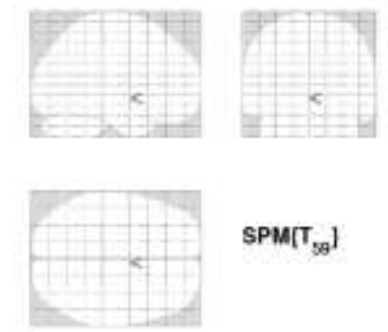

SPM[T $\left.T_{58}\right]$

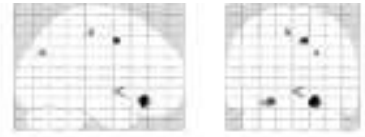

$\operatorname{SPM}\left(\mathrm{T}_{59}\right)$

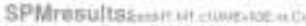

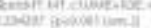

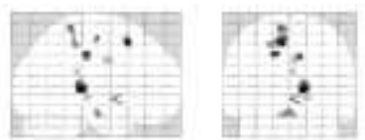

$\operatorname{SPM}\left(\mathrm{T}_{\mathrm{sp}}\right)$

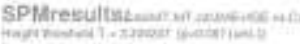

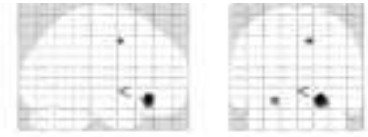

$\left.\operatorname{SPM}_{\text {sq }}\right]$

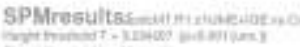
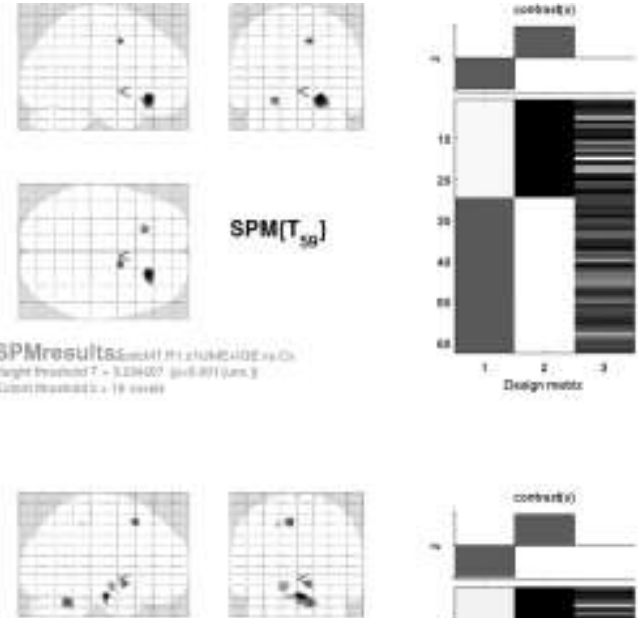

$\operatorname{sPM}\left[T_{g_{0}}\right]$

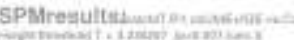

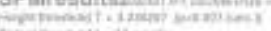

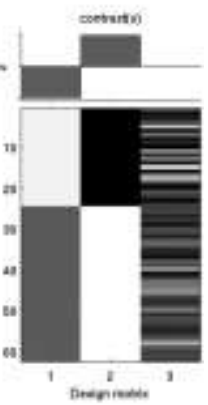

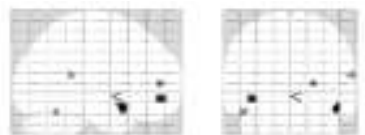

SPM[T spa $_{2}$

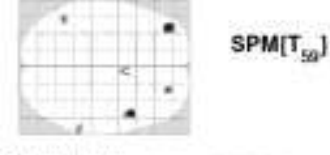

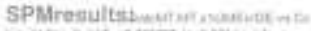

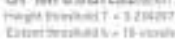
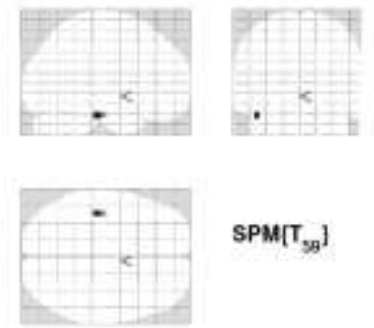

$\operatorname{SPM}\left[T_{S B}\right]$

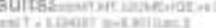
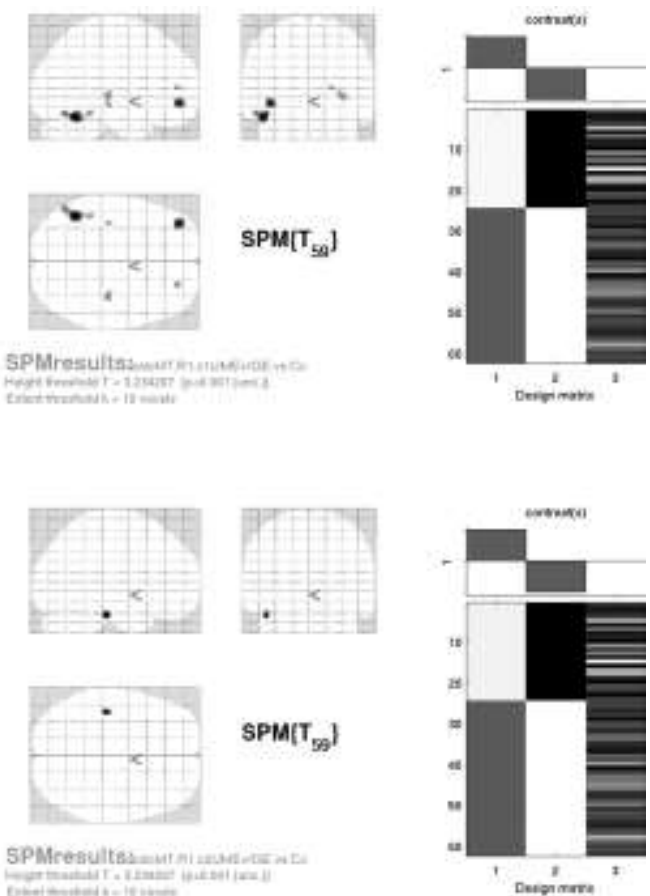

Abbildung 2: JME und IGE > Cx (A graue Substanz, B weiße Substanz)

\subsubsection{Vergleich der IGE-Patienten mit der Kontrollgruppe}

Die Analyse detektierte verschiedene Volumenabnahmen in der grauen Substanz bei der Patientengruppe IGE ( $p<0,001$, unkorrigiert, vergleiche Abbildung 3). Diese fanden sich in allen drei Untersuchungen im linken Gyrus fusiformis (hrT1, calcMT.MT -24 -78 -6, 
calcMT.R1: -24 -78 -5), im linken (hrT1, calcMT.MT: -22 27 -11, calcMT.R1: -22 $26-11$ ) sowie im rechten frontoorbitalen Kortex (hrT1: 2730 -14, calcMT.MT: 2432 -12, calcMT.R1: 2432 -11) und im rechten Frontalpol (hrT1, calcMT.MT: 3936 -9, calcMT.R1: 3836 -8). Kleinere Cluster zeigten sich weiter im rechten Gyrus postcentralis (hrT1: 48 -18 45) sowie im rechten okzipitalen Kortex (calcMT.MT: 24 -75 37).

\begin{tabular}{|c|c|c|c|c|c|c|c|}
\hline & $p$ (FWE) & p(FWE/cluster) & $\mathbf{k}$ & $\mathbf{T}$ & \multicolumn{3}{|c|}{ MNI* } \\
\hline \multirow[t]{5}{*}{ hrT1 } & 0,306 & 0,673 & 174 & 4,52 & -24 & -78 & -6 \\
\hline & 0,587 & 0,328 & 333 & 4,19 & 27 & 30 & -14 \\
\hline & 0,848 & 0,964 & 49 & 3,88 & -22 & 27 & -11 \\
\hline & 0,937 & 0,995 & 13 & 3,72 & 48 & -18 & 45 \\
\hline & 0,969 & 0,997 & 10 & 3,62 & 39 & 36 & -9 \\
\hline \multirow[t]{6}{*}{ calcMT.MT } & 0,068 & 0,061 & 662 & 5,12 & 24 & 32 & -12 \\
\hline & 0,921 & & & 3,78 & 39 & 36 & -9 \\
\hline & 0,423 & 0,807 & 122 & 4,39 & -24 & -78 & -6 \\
\hline & 0,495 & 0,281 & 352 & 4,30 & -22 & 27 & -11 \\
\hline & 0,701 & & & 4,08 & -34 & 36 & -15 \\
\hline & 0,996 & 0,994 & 17 & 3,44 & 24 & -75 & 37 \\
\hline \multirow[t]{4}{*}{ calcMT.R1 } & 0,083 & 0,064 & 664 & 5,06 & 24 & 32 & -11 \\
\hline & 0,461 & & & 4,34 & 38 & 36 & -8 \\
\hline & 0,533 & 0,820 & 120 & 4,26 & -22 & 26 & -11 \\
\hline & 0,613 & 0,923 & 76 & 4,18 & -24 & -78 & -5 \\
\hline
\end{tabular}

Tabelle 8: IGE < Cx (graue Substanz)

Innerhalb der weißen Substanz fanden sich kleine Cluster $(p<0,001$, unkorrigiert, vergleiche Abbildung 3) im Bereich des linken Gyrus frontalis superior (hrT1: -4 18 54), des rechten Thalamus (hrT1: 10 -27 6) sowie im Bereich des Hirnstamms (calcMT.R1: -3 -46 -72).

\begin{tabular}{|c|c|c|c|c|c|c|c|}
\hline & p(FWE) & $\mathrm{p}$ (FWE/cluster) & $\mathbf{k}$ & $\mathbf{T}$ & \multicolumn{3}{|c|}{ MNI* } \\
\hline hrT1 & 0,887 & 0,937 & 28 & 3,62 & -4 & 18 & 54 \\
\hline & 0,947 & 0,950 & 22 & 3,49 & 10 & -27 & 6 \\
\hline calcMT.MT & \multicolumn{7}{|c|}{ No suprathreshold cluster } \\
\hline calcMT.R1 & 0,979 & 0,951 & 25 & 3,40 & -3 & -46 & -72 \\
\hline
\end{tabular}

Tabelle 9: IGE < Cx (weiße Substanz)

Bei der Analyse hinsichtlich der Volumenzunahme von grauer Substanz fanden sich bei den Daten aus den drei Untersuchungen insbesondere im linken Gyrus temporalis inferior bzw. 
im linken Gyrus fusiformis größere Cluster $(\mathrm{p}<0,001$, unkorrigiert, vergleiche Abbildung 4, hrT1: -45 -40 -14, -48 -54 -21, -51 -28 -30, -52 -34 -23, calcMT.MT: -54 -31 -33, -58 -40 -30, -46 -40 -14, calcMT.R1: $-45-54-20,-45-40-14)$. Basierend auf der quantitativen MRTBildgebung zeigten sich noch kleinere Cluster im Bereich des rechten Temporalpols (calcMT.MT: 4712 -14), des rechten Gyrus supramarginalis (calcMT.MT: 63 -39 18) sowie im Bereich des linken Frontalpols (calcMT.R1: -38 50 -5) und des rechten Gyrus fusiformis (calcMT.R1: 6 -87 -26).

\begin{tabular}{|c|c|c|c|c|c|c|c|}
\hline & $p$ (FWE) & p(FWE/cluster) & $\mathbf{k}$ & $\mathbf{T}$ & \multicolumn{3}{|c|}{$\mathrm{MNI}^{*}$} \\
\hline \multirow[t]{4}{*}{ hrT1 } & 0,202 & 0,448 & 267 & 4,70 & -45 & -40 & -14 \\
\hline & 0,870 & & & 3,85 & -48 & -54 & -21 \\
\hline & 0,962 & 0,867 & 100 & 3,65 & -51 & -28 & -30 \\
\hline & 0,967 & & & 3,63 & -52 & -34 & -23 \\
\hline \multirow[t]{6}{*}{ calcMT.MT } & 0,836 & 0,907 & 82 & 3,92 & -54 & -31 & -33 \\
\hline & 0,997 & & & 3,42 & -58 & -40 & -30 \\
\hline & 0,851 & 0,967 & 48 & 3,90 & -46 & -40 & -14 \\
\hline & 0,900 & 0,964 & 50 & 3,82 & 47 & 12 & -14 \\
\hline & 0,936 & 0,967 & 48 & 3,74 & -48 & -54 & -21 \\
\hline & 0,995 & 0,988 & 27 & 3,45 & 63 & -39 & 18 \\
\hline \multirow[t]{4}{*}{ calcMT.R1 } & 0,277 & 0,467 & 256 & 4,58 & -45 & -54 & -20 \\
\hline & 0,828 & & & 3,93 & -45 & -40 & -14 \\
\hline & 0,865 & 0,943 & 65 & 3,88 & -38 & 50 & -5 \\
\hline & 0,995 & 0,997 & 10 & 3,45 & 6 & -87 & -26 \\
\hline
\end{tabular}

Tabelle 10: IGE > Cx (graue Substanz)

Bezüglich der weißen Substanz zeigten sich hauptsächlich zwei Bereiche mit einer Volumenzunahme bei der Patientengruppe ( $p<0,001$, unkorrigiert, vergleiche Abbildung 4). Hierzu zählen der linke Gyrus temporalis inferior bzw. der linke Gyrus fusiformis (hrT1: -42 -25 -23, calcMT.MT: $-44-24-21,-54-52-11$, calcMT.R1: -42 -24 -23) und der rechte Precuneus (hrT1: 6 -61 46, calcMT.MT: 8 -60 45, 4 -60 36).

\begin{tabular}{|l|c|c|c|c|rrr|}
\hline & $\mathbf{p}$ (FWE) & $\mathbf{p}$ (FWE/cluster) & $\mathbf{k}$ & $\mathbf{4}$ & \multicolumn{3}{|c|}{ MNI $^{*}$} \\
\hline hrT1 & 0,248 & 0,853 & 61 & 4,43 & -42 & -25 & -23 \\
& 0,964 & 0,971 & 11 & 3,43 & 6 & -61 & 46 \\
\hline calcMT.MT & 0,582 & 0,629 & 138 & 4,05 & 8 & -60 & 45 \\
& 0,678 & & & 3,94 & 4 & -60 & 36 \\
\hline calcMT.MT & 0,822 & 0,863 & 62 & 3,77 & -44 & -24 & -21 \\
& 0,935 & 0,958 & 24 & 3,58 & -54 & -52 & -11 \\
\hline
\end{tabular}

Tabelle 11: IGE > Cx (weiße Substanz) 


\begin{tabular}{l|c|c|c|c|c|}
\hline & $\mathbf{p}(\mathrm{FWE})$ & $\mathbf{p}(\mathrm{FWE} /$ cluster) & $\mathbf{k}$ & $\mathbf{T}$ & \multicolumn{2}{|c|}{ MNI* $^{*}$} \\
\hline calcMT.R1 & 0,730 & 0,919 & 39 & 3,87 & $-42 \quad-24 \quad-23$ \\
\hline $\begin{array}{l}\text { * ermittelte Koordinaten mit } \mathrm{p}<0,001, \text { unkorrigiert bezüglich des peak level-sowie des } \\
\text { spatial extent family wise errors }\end{array}$
\end{tabular}

Tabelle 11: IGE > Cx (weiße Substanz), fortgesetzt

A
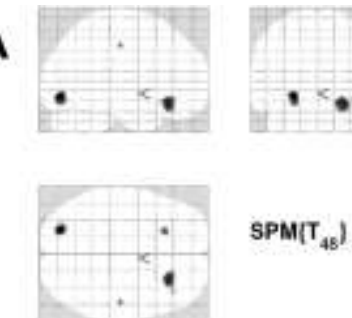

SPHAresultsh mavtusine ino

B
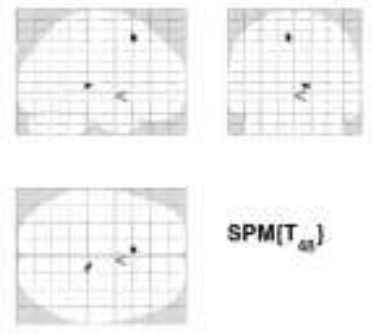

SPMIT $\left._{a n}\right]$

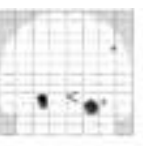

$M\left(T_{48}\right)$

SPMrosultshanawh wasticic

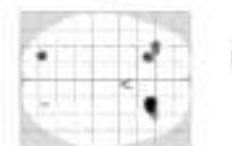

$\left.\operatorname{SPM}_{48}\right]$

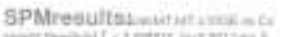

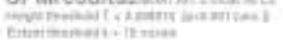

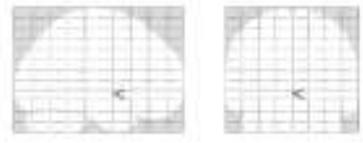

$\left.\mathrm{SPM}_{40}\right]$

SPMrosultasminecut anut -

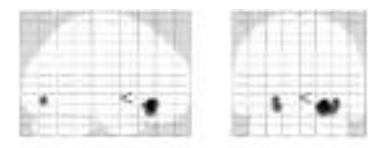

$\left.\operatorname{SPM}_{48}\right]$

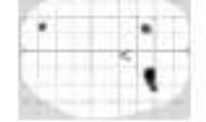

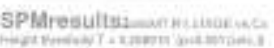
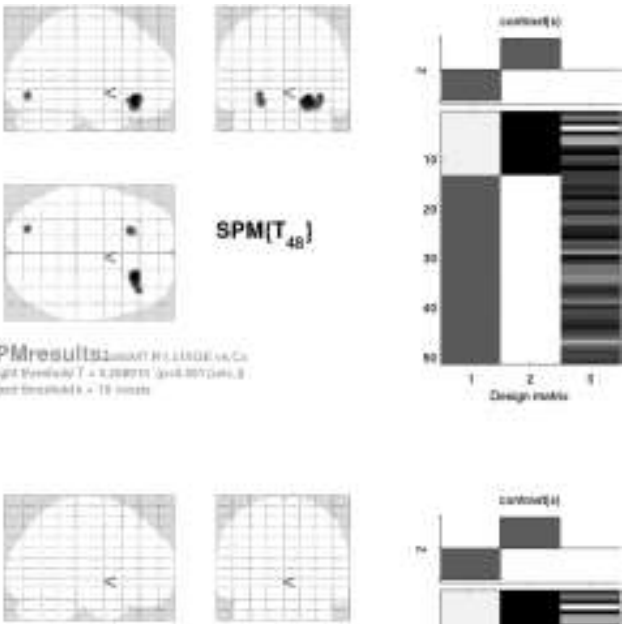

$\operatorname{SPM}_{4 n}$
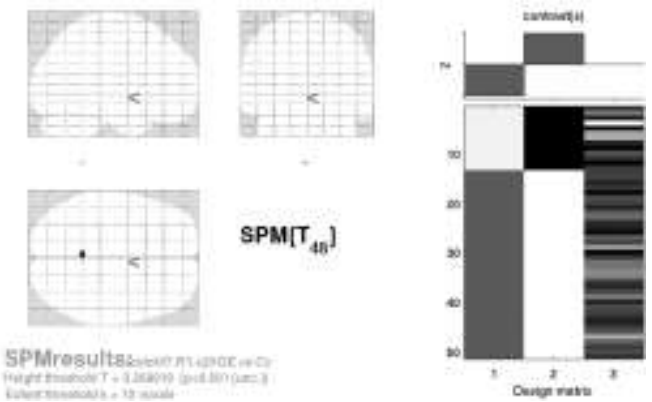

Abbildung 3: IGE < Cx (A graue Substanz, B weiße Substanz)

A
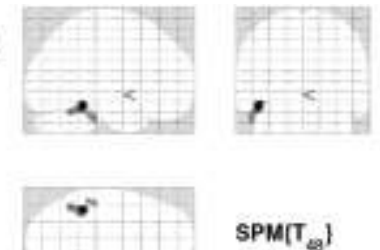

SPMresultsinanitions -

B
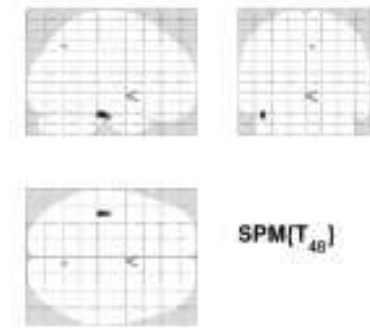

$\left.\operatorname{SPM}_{48}\right]$

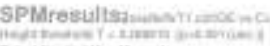

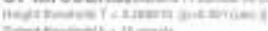

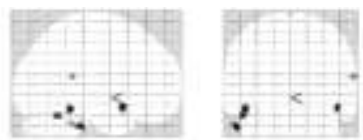

SPMIT

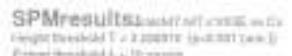
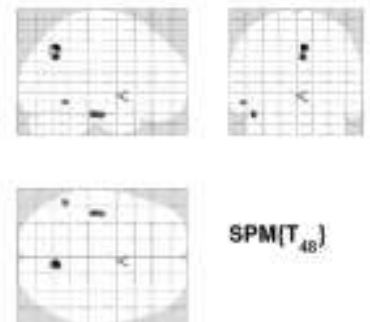

$\left.\operatorname{SPM}_{48}\right)$

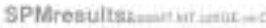

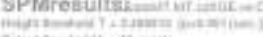

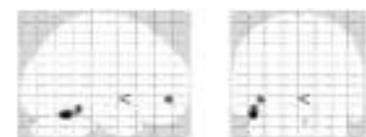

SPMIT $\left.T_{43}\right)$
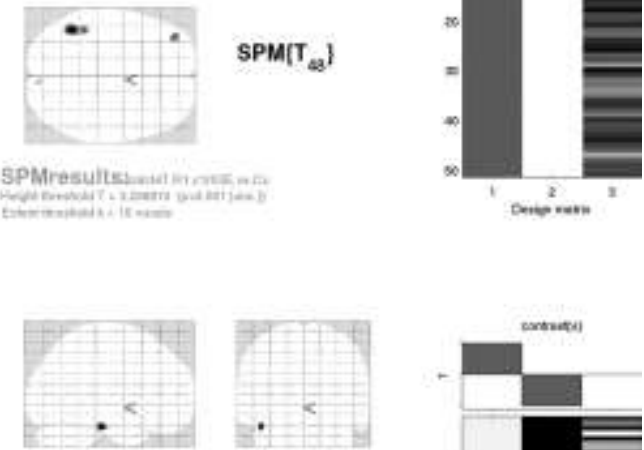

$\operatorname{SPM}\left[T_{48}\right]$

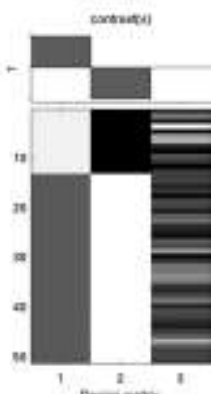

Abbildung 4: IGE > Cx (A graue Substanz, B weiße Substanz) 


\subsubsection{Vergleich der JME-Patienten mit der Kontrollgruppe}

Die VBM der Daten aus der T1-gewichteten Sequenz sowie aus der MT-Karte und der T1Relaxometrie zeigte im Vergleich der Volumina der grauen Substanzen zwischen der Gruppe JME und der Kontrollgruppe Cx mehrere kleinere zerebrale Bereiche, in denen sich relativ ein geringeres Volumen befindet ( $p<0,001$, unkorrigiert, vergleiche Abbildung 5 ). Hierzu zählt der Bereich des prä- (hrT1: 0 -19 52, calcMT.MT: 0 -18 54, calcMT.R1: 2 -15 58) und des postzentralen Gyrus (hrT1: -14 -48 61), des linken Gyrus fusiformis (hrT1: -39 -70 -12, calcMT.MT: -40 -72 -14) sowie verschiedene weitere Cluster (vergleiche Tabelle 12).

\begin{tabular}{|c|c|c|c|c|c|c|c|}
\hline & $p$ (FWE) & p(FWE/cluster) & $\mathbf{k}$ & $\mathbf{T}$ & \multicolumn{3}{|c|}{$\mathrm{MNI}^{*}$} \\
\hline \multirow[t]{5}{*}{ hrT1 } & 0,722 & 0,980 & 35 & 4,05 & 40 & -25 & -8 \\
\hline & 0,930 & 0,995 & 13 & 3,75 & -14 & -48 & 61 \\
\hline & 0,946 & 0,960 & 52 & 3,71 & 0 & -19 & 52 \\
\hline & 0,952 & 0,995 & 13 & 3,69 & -39 & -70 & -12 \\
\hline & 0,995 & 0,997 & 10 & 3,45 & 35 & -1 & -11 \\
\hline \multirow[t]{3}{*}{ calcMT.MT } & 0,862 & 0,933 & 69 & 3,89 & -33 & -6 & -9 \\
\hline & 0,879 & 0,867 & 99 & 3,87 & 0 & -18 & 54 \\
\hline & 0,947 & 0,950 & 59 & 3,73 & -40 & -72 & -14 \\
\hline \multirow[t]{5}{*}{ calcMT.R1 } & 0,856 & 0,993 & 20 & 3,91 & 57 & 24 & 25 \\
\hline & 0,860 & 0,996 & 14 & 3,91 & 40 & -27 & -6 \\
\hline & 0,984 & 0,997 & 11 & 3,58 & 15 & 3 & 43 \\
\hline & 0,985 & 0,991 & 24 & 3,58 & -26 & 2 & 67 \\
\hline & 0,996 & 0,997 & 11 & 3,45 & 2 & -15 & 58 \\
\hline
\end{tabular}

Tabelle 12: JME < Cx (graue Substanz)

Im Bereich der weißen Substanz fanden sich in der Analyse der Daten aus allen drei Untersuchungen ( $p<0,001$, unkorrigiert, vergleiche Abbildung 5 ) vor allem Cluster im Bereich des linken pre- (hrT1: -10 -24 48, -8 -30 55, calcMT.MT: -15 -22 43, calcMT.R1 -9 -25 46) sowie des linken postzentralen Gyrus (hrT1: -15 -37 49, calcMT.MT: -15 -36 48, -9 -40 67, calcMT.R1: -14 -37 49), des linken Precuneus (hrT1: -12 -46 46), der Thalami (hrT1: 15 -28 6, 20 -25 -5, calcMT.MT: 14 -28 6, calcMT.R1: -14 -24 15), des rechten frontalen Operculums (hrT1: 4412 6, calcMT.MT: 4512 6, calcMT.R1: 4412 6) und des linken Gyrus frontalis inferior (hrT1: -52 27 7, calcMT.R1: -52 27 7). In der Untersuchung der MT-Karte zeigten sich weiter Cluster im Bereich der linken supplementär-motorischen Rinde des Motorkortex (MNI: -15 -7 45) sowie im linken Gyrus paracinguli (MNI: -8 23 42). Weitere detektierte Cluster finden sich in Tabelle 13. 


\begin{tabular}{|c|c|c|c|c|c|c|c|}
\hline & $p$ (FWE) & $\mathrm{p}$ (FWE/cluster) & k & $\mathbf{T}$ & \multicolumn{3}{|c|}{ MNI* } \\
\hline \multirow[t]{12}{*}{ hrT1 } & 0,398 & 0,254 & 332 & 4,23 & -15 & -37 & 49 \\
\hline & 0,862 & & & 3,67 & -12 & -46 & 46 \\
\hline & 0,554 & 0,776 & 88 & 4,04 & 4 & -3 & -15 \\
\hline & 0,703 & 0,680 & 121 & 3,88 & -9 & -7 & 21 \\
\hline & 0,984 & & & 3,34 & 0 & 2 & 18 \\
\hline & 0,929 & 0,867 & 56 & 3,54 & 15 & -28 & 6 \\
\hline & 0,930 & 0,954 & 20 & 3,54 & -52 & 27 & 7 \\
\hline & 0,954 & 0,950 & 22 & 3,47 & 44 & 12 & 6 \\
\hline & 0,963 & 0,958 & 18 & 3,44 & -8 & 18 & 52 \\
\hline & 0,970 & 0,958 & 18 & 3,41 & 20 & -25 & -5 \\
\hline & 0,970 & 0,923 & 34 & 3,41 & -10 & -24 & 48 \\
\hline & 0,973 & 0,965 & 14 & 3,40 & -8 & -30 & 55 \\
\hline \multirow[t]{12}{*}{ calcMT.MT } & 0,598 & 0,032 & 727 & 4,05 & -15 & -36 & 48 \\
\hline & 0,628 & & & 4,02 & -15 & -22 & 43 \\
\hline & 0,747 & & & 3,88 & -12 & -46 & 46 \\
\hline & 0,685 & 0,890 & 53 & 3,95 & -52 & 26 & 7 \\
\hline & 0,841 & 0,901 & 49 & 3,76 & -9 & -40 & 67 \\
\hline & 0,859 & 0,792 & 86 & 3,73 & 14 & -28 & 6 \\
\hline & 0,860 & 0,919 & 42 & 3,73 & -8 & 23 & 42 \\
\hline & 0,886 & 0,764 & 95 & 3,69 & 45 & 12 & 6 \\
\hline & 0,916 & 0,873 & 59 & 3,63 & -3 & -3 & 19 \\
\hline & 0,950 & 0,953 & 27 & 3,55 & -10 & 18 & 52 \\
\hline & 0,976 & 0,951 & 28 & 3,45 & 9 & -46 & -30 \\
\hline & 0,985 & 0,957 & 25 & 3,40 & -15 & -7 & 45 \\
\hline \multirow[t]{11}{*}{ calcMT.R1 } & 0,689 & 0,345 & 253 & 3,93 & -14 & -24 & 15 \\
\hline & 0,715 & & & 3,90 & -14 & -13 & 22 \\
\hline & 0,955 & & & 3,51 & -20 & -33 & 12 \\
\hline & 0,766 & 0,528 & 171 & 3,84 & -14 & -37 & 49 \\
\hline & 0,784 & 0,213 & 344 & 3,82 & 14 & -49 & -29 \\
\hline & 0,943 & 0,942 & 29 & 3,55 & -12 & -46 & 46 \\
\hline & 0,966 & 0,630 & 135 & 3,47 & -18 & -57 & -29 \\
\hline & 0,972 & & & 3,45 & -9 & -55 & -36 \\
\hline & 0,971 & 0,961 & 20 & 3,45 & -9 & -25 & 46 \\
\hline & 0,971 & 0,976 & 12 & 3,45 & -52 & 27 & 7 \\
\hline & 0,972 & 0,977 & 11 & 3,45 & 44 & 12 & 6 \\
\hline
\end{tabular}

Tabelle 13: JME < Cx (weiße Substanz)

Ein Vergleich der Daten hinsichtlich der Areale der grauen Substanz, deren Volumina bei der Patientengruppe größer erschienen, zeigte vor allem einige Cluster $(p<0,001$, unkorrigiert, vergleiche Abbildung 6) im Bereich des rechten parietalen Operculums (hrT1: 52 -34 31, calcMT.R1: 51 -34 31), des rechten Frontal- (calcMT.MT: 4141 -5, 2759 -9, calcMT.R1: 24 
62 -9) und des rechten Temporalpols (hrT1: 2714 -29, 3515 -26). Weitere Cluster fanden sich im okzipitalen Kortex sowie vereinzelt in anderen Bereichen (vergleiche Tabelle 14).

\begin{tabular}{|c|c|c|c|c|c|c|c|}
\hline & $p$ (FWE) & p(FWE/cluster) & k & $\mathbf{T}$ & \multicolumn{3}{|c|}{ MNI* } \\
\hline \multirow[t]{6}{*}{ hrT1 } & 0,710 & 0,917 & 77 & 4,07 & 52 & -34 & 31 \\
\hline & 0,810 & 0,927 & 72 & 3,95 & 27 & 14 & -29 \\
\hline & 0,993 & & & 3,47 & 35 & 15 & -26 \\
\hline & 0,910 & 0,867 & 100 & 3,79 & 14 & -94 & -18 \\
\hline & 0,970 & 0,993 & 18 & 3,63 & 51 & -60 & -15 \\
\hline & 0,982 & 0,991 & 21 & 3,57 & 34 & -22 & 0 \\
\hline \multirow[t]{9}{*}{ calcMT.MT } & 0,665 & 0,839 & 110 & 4,14 & 52 & -34 & 31 \\
\hline & 0,830 & 0,980 & 36 & 3,94 & 6 & -42 & 75 \\
\hline & 0,897 & 0,940 & 65 & 3,84 & 34 & -22 & 3 \\
\hline & 0,901 & 0,984 & 32 & 3,83 & 45 & -6 & -33 \\
\hline & 0,946 & 0,992 & 21 & 3,73 & 41 & 41 & -5 \\
\hline & 0,979 & 0,979 & 37 & 3,61 & -39 & 48 & -8 \\
\hline & 0,986 & 0,975 & 41 & 3,57 & 27 & 59 & -9 \\
\hline & 0,990 & 0,990 & 24 & 3,53 & -45 & -78 & 16 \\
\hline & 0,997 & 0,992 & 21 & 3,43 & 18 & -36 & 67 \\
\hline \multirow[t]{5}{*}{ calcMT.R1 } & 0,475 & 0,864 & 103 & 4,35 & 51 & -34 & 31 \\
\hline & 0,925 & 0,937 & 69 & 3,79 & 34 & -22 & 1 \\
\hline & 0,925 & 0,991 & 25 & 3,79 & 32 & -78 & -5 \\
\hline & 0,991 & 0,986 & 32 & 3,53 & -45 & -76 & 16 \\
\hline & 0,994 & 0,993 & 20 & 3,49 & 24 & 62 & -9 \\
\hline
\end{tabular}

Tabelle 14: JME > Cx (graue Substanz)

Die Analyse der weißen Substanz erbrachte hinsichtlich einer Zunahme der Volumina keine größeren Unterschiede ( $p<0,001$, unkorrigiert, vergleiche Abbildung 6). Vereinzelt fand sich neben einzelnen kleineren Arealen ein Cluster im Bereich des latero-okzipitalen Kortex links (calcMT.MT: -34 -87 3, calcMT.R1: -33 -85 3).

\begin{tabular}{|l|c|c|c|c|rrr|}
\hline & p(FWE) & p(FWE/cluster) & k & T & \multicolumn{3}{c|}{ MNI* $^{*}$} \\
\hline hrT1 & \multicolumn{7}{|c|}{ No suprathreshold cluster } \\
\hline calcMT.MT & 0,463 & 0,903 & 48 & 4,20 & -34 & -87 & 3 \\
\hline calcMT.R1 & 0,534 & 0,951 & 25 & 4,10 & 50 & -46 & 22 \\
& 0,840 & 0,972 & 14 & 3,74 & 32 & 56 & -3 \\
& 0,892 & 0,967 & 17 & 3,66 & -33 & -85 & 3 \\
& 0,916 & 0,970 & 15 & 3,61 & -15 & -75 & -5 \\
\hline & * ermittelte Koordinaten mit p 0,001, unkorrigiert bezüglich des peak level- sowie des \\
spatial extent family wise errors
\end{tabular}

Tabelle 15: JME > Cx (weiße Substanz) 
A
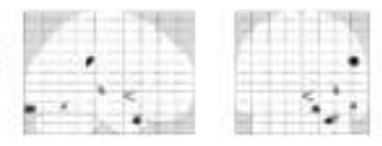

$\operatorname{SPM}\left(T_{46}\right)$

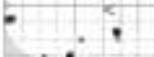

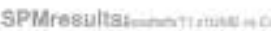

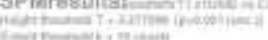

B
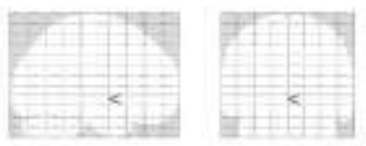

$\operatorname{SPM}\left[T_{48}\right]$

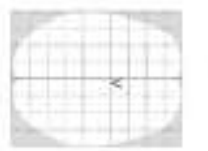

SPMresultainantiandis

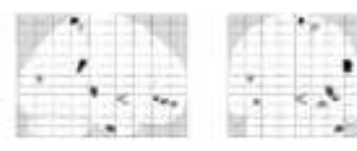

$\left.\operatorname{SPM} \mathrm{TT}_{46}\right)$

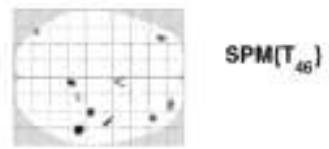

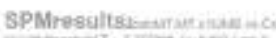

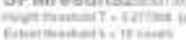
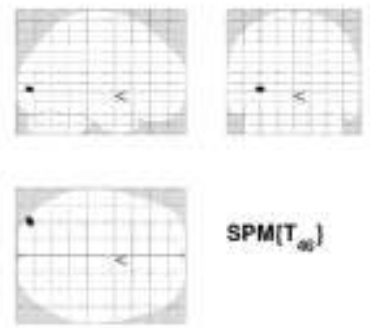

SPM[T $\left.T_{\infty}\right]$

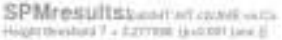

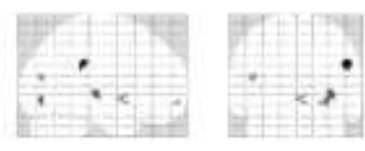

$\operatorname{SPM}\left(T_{46}\right)$

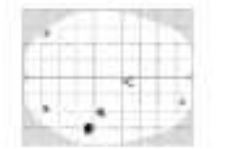

SPMrosultaknom monat wo

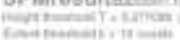
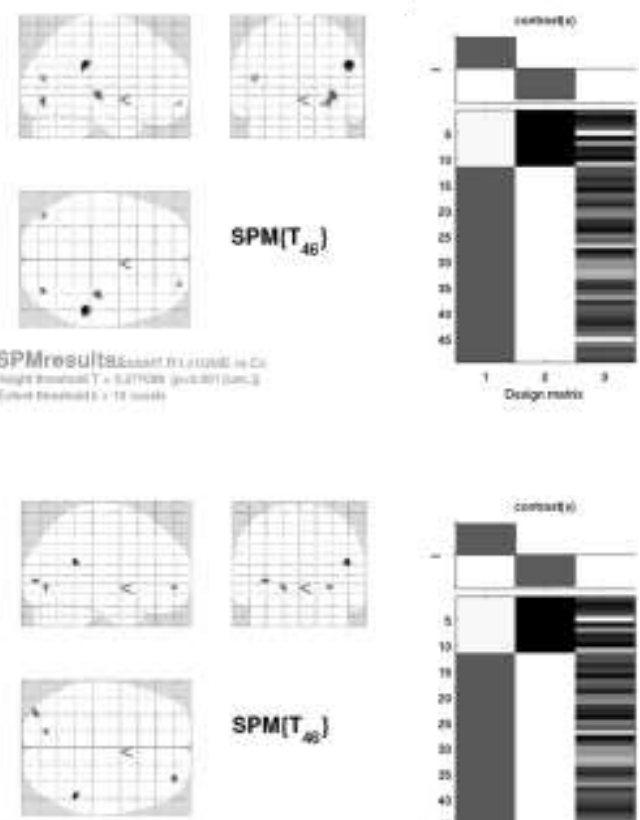

SPM[T $\left.T_{48}\right]$

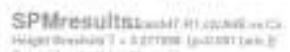

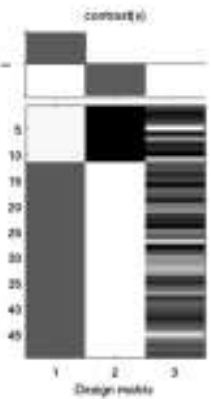

Abbildung 5: JME < Cx (A graue Substanz, B weiße Substanz)

A
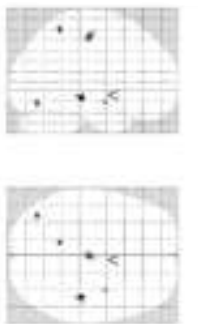

SPM[T $\left._{48}\right]$

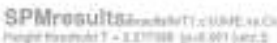

B
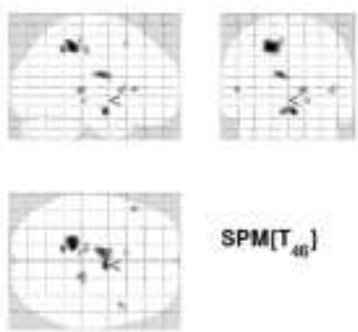

$\left.\mathrm{SPM}_{40}\right]$

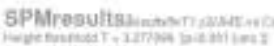
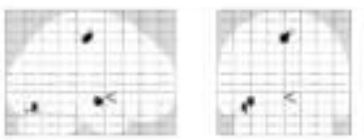

$\left.\operatorname{SPM}_{46}\right)$
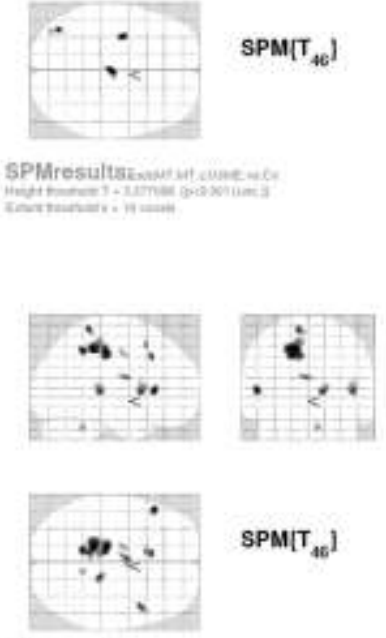

SPMresultaknimatamenco

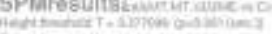
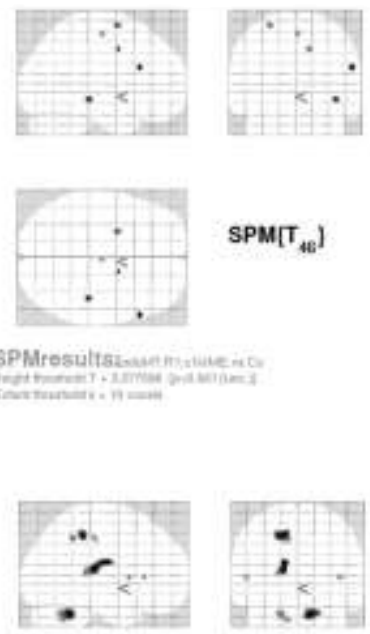

SPM[T 4 I
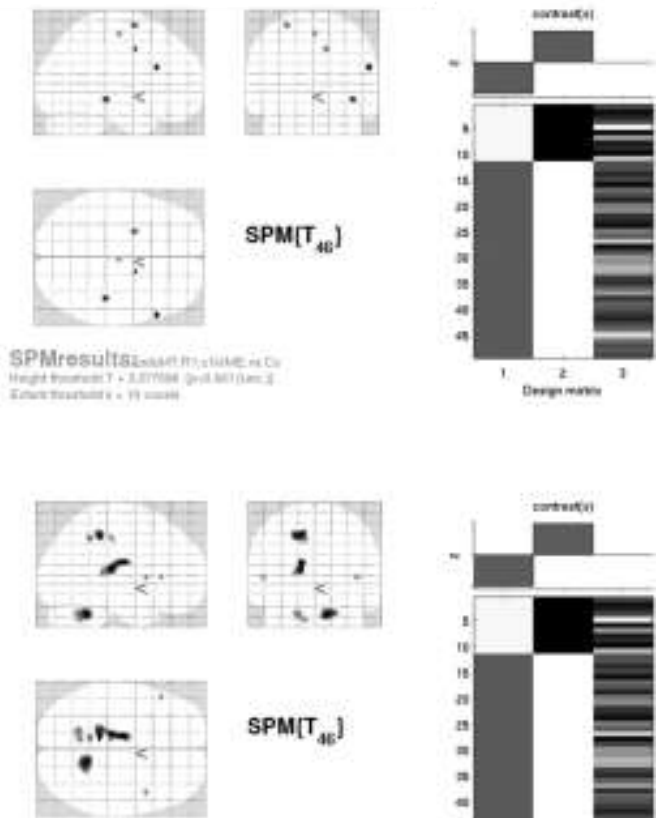

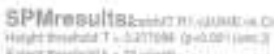

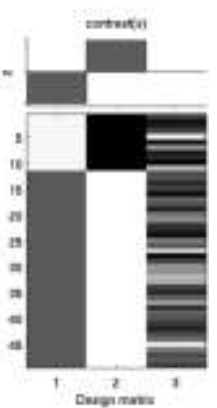

Abbildung 6: JME > Cx (A graue Substanz, B weiße Substanz)

\subsubsection{Vergleich der IGE-Patienten mit den JME-Patienten}

Der direkte Vergleich beider Patientengruppen miteinander zeigte auf unkorrigiertem Signifikanzniveau von $p<0,001$ (vergleiche Abbildung 7) dezente strukturelle Unterschiede 
in der grauen Substanz. Die Volumenabnahmen im Vergleich von JME zu IGE fanden sich jedoch nur in den quantitativen Karten. Betroffen waren unter anderem Bereiche im rechten präzentralen Gyrus (calcMT.R1: 2 -15 58) sowie im Gyrus frontalis superior und inferior (calcMT.R1: 5724 25, -26 2 67).

\begin{tabular}{|l|c|c|c|c|c|cr|}
\hline & $\mathbf{p}(\mathrm{FWE})$ & $\mathbf{p}(\mathrm{FWE} /$ cluster) & $\mathbf{k}$ & $\mathbf{T}$ & \multicolumn{3}{c|}{ MNI* $^{*}$} \\
\hline hrT1 & \multicolumn{6}{|c|}{ No suprathreshold cluster } \\
\hline calcMT.MT & 0,443 & 0,746 & 138 & 5,11 & -36 & -12 & -9 \\
& 0,964 & & & 4,13 & -28 & -21 & -6 \\
\hline calcMT.R1 & 0,856 & 0,993 & 20 & 3,91 & 57 & 24 & 25 \\
& 0,860 & 0,996 & 14 & 3,91 & 40 & -27 & -6 \\
& 0,984 & 0,997 & 11 & 3,58 & 15 & 3 & 43 \\
& 0,985 & 0,991 & 24 & 3,58 & -26 & 2 & 67 \\
& 0,996 & 0,997 & 11 & 3,45 & 2 & -15 & 58 \\
\hline
\end{tabular}

Tabelle 16: JME < IGE (graue Substanz)

Innerhalb der weißen Substanz konnten in allen drei Sequenzen/Karten kleinere Cluster detektiert werden ( $p<0,001$, unkorrigiert). Diese fanden sich unter anderem im linken postzentralen (hrT1: -14 -37 49, calcMT.R1: -12 -37 49) sowie im linken und rechten präzentralen Gyrus (calcMT.MT: 12 -15 55, -10 -36 49).

\begin{tabular}{|c|c|c|c|c|c|c|c|}
\hline & $p$ (FWE) & p(FWE/cluster) & k & $\mathbf{T}$ & \multicolumn{3}{|c|}{$\mathrm{MNI}^{*}$} \\
\hline \multirow[t]{2}{*}{ hrT1 } & 0,670 & 0,922 & 41 & 4,46 & 12 & -49 & 45 \\
\hline & 0,672 & 0,979 & 13 & 4,46 & -14 & -37 & 49 \\
\hline \multirow[t]{4}{*}{ calcMT.MT } & 0,455 & 0,841 & 72 & 4,88 & 12 & -15 & 55 \\
\hline & 0,508 & 0,748 & 98 & 4,79 & 15 & -49 & 43 \\
\hline & 0,820 & 0,983 & 16 & 4,30 & -10 & -36 & 49 \\
\hline & 0,884 & 0,871 & 63 & 4,17 & 14 & -66 & 46 \\
\hline \multirow[t]{2}{*}{ calcMT.R1 } & 0,466 & 0,819 & 76 & 4,81 & 12 & -51 & 45 \\
\hline & 0,707 & 0,973 & 19 & 4,44 & -12 & -37 & 49 \\
\hline
\end{tabular}

Tabelle 17: JME < IGE (weiße Substanz)

Der Vergleich bezüglich einer Volumenzunahme von JME relativ zur Gruppe IGE zeigte bei unkorrigiertem Signifikanzniveau etwas stärkere Alterationen innerhalb der grauen Substanz (vergleiche Abbildung 8). Die Cluster konnten vor allem im Gyrus fusiformis (hrT1: -32 -70 -5, -26 -84 -9, calcMT.MT: -32 -72 -5, -27 -84 -9, calc MT.R1: -30 -70 -5, -26 -84 -9) sowie im fronto-orbitalen Kortex (hrT1: -22 15 -29, 2424 -12, calcMT.MT: $2426-12,3033-9$ ) detektiert werden. 


\begin{tabular}{|c|c|c|c|c|c|c|c|}
\hline & $p$ (FWE) & $\mathrm{p}$ (FWE/cluster) & k & $\mathbf{T}$ & \multicolumn{3}{|c|}{$\mathrm{MNI}^{*}$} \\
\hline \multirow[t]{6}{*}{ hrT1 } & 0,336 & 0,737 & 145 & 5,27 & -32 & -70 & -5 \\
\hline & 0,994 & & & 3,85 & -26 & -84 & -9 \\
\hline & 0,535 & 0,866 & 100 & 4,92 & -22 & 15 & -29 \\
\hline & 0,903 & 0,996 & 18 & 4,30 & 24 & 24 & -12 \\
\hline & 0,910 & 0,993 & 24 & 4,28 & -38 & -57 & 42 \\
\hline & 0,999 & 0,998 & 10 & 3,68 & -4 & -99 & -3 \\
\hline \multirow[t]{7}{*}{ calcMT.MT } & 0,157 & 0,650 & 168 & 5,78 & -32 & -72 & -5 \\
\hline & 0,416 & & & 5,15 & -27 & -84 & -9 \\
\hline & 0,837 & 0,968 & 51 & 4,48 & -15 & 42 & -14 \\
\hline & 0,849 & 0,978 & 43 & 4,45 & -38 & -57 & 42 \\
\hline & 0,947 & 0,992 & 27 & 4,21 & 24 & 26 & -12 \\
\hline & 0,985 & 0,995 & 21 & 3,99 & 18 & -85 & 45 \\
\hline & 0,986 & 0,920 & 77 & 3,99 & 30 & 33 & -9 \\
\hline \multirow[t]{6}{*}{ calcMT.R1 } & 0,287 & 0,829 & 114 & 5,41 & -30 & -70 & -5 \\
\hline & 0,916 & 0,981 & 41 & 4,30 & -14 & 42 & -12 \\
\hline & 0,980 & & & 4,04 & -15 & 50 & -9 \\
\hline & 0,937 & 0,998 & 12 & 4,24 & -26 & -84 & -9 \\
\hline & 0,966 & 0,876 & 97 & 4,12 & 27 & 28 & -17 \\
\hline & 0,993 & 0,998 & 12 & 3,89 & -38 & -57 & 42 \\
\hline
\end{tabular}

Tabelle 18: JME > IGE (graue Substanz)

Bezüglich der weißen Substanz fanden sich Volumenzunahmen in verschiedenen kleineren Clustem ( $p<0,001$, unkorrigiert). Betroffen waren insbesondere der rechte Okzipitalpol (calcMT.MT: 21 -94 -9) und der linke präzentrale Gyrus (calcMT.R1: -51 0 37).

\begin{tabular}{|l|c|c|c|c|cr|r|}
\hline & $\mathbf{p}(\mathrm{FWE})$ & $\mathbf{p}(\mathrm{FWE} /$ cluster) & $\mathbf{k}$ & $\mathbf{T}$ & \multicolumn{3}{|c|}{ MNI* $^{*}$} \\
\hline hrT1 & \multicolumn{7}{|c|}{ No suprathreshold cluster } \\
\hline calcMT.MT & 0,924 & 0,974 & 22 & 4,07 & 45 & -72 & -12 \\
& 0,972 & 0,980 & 18 & 3,88 & -14 & -84 & 0 \\
& 0,974 & 0,919 & 47 & 3,87 & 21 & -94 & -9 \\
& 0,986 & 0,980 & 18 & 3,78 & -16 & -75 & -5 \\
\hline calcMT.R1 & 0,861 & 0,975 & 18 & 4,18 & -51 & 0 & 37 \\
\hline * ermittelte Koordinaten mit p 0,001, unkorrigiert bezüglich des peak level- sowie des \\
spatial extent family wise errors
\end{tabular}

Tabelle 19: JME > IGE (weiße Substanz) 
A
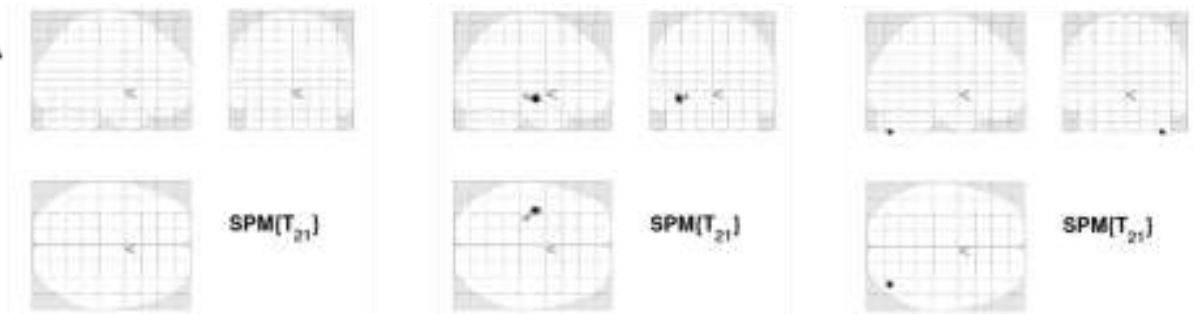

SPM[T $\left._{21}\right]$

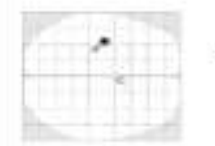

$\operatorname{SPM}\left[T_{21}\right]$

SPMIT $\left.T_{21}\right]$

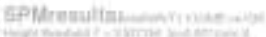

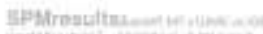

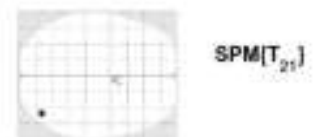

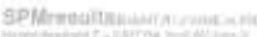

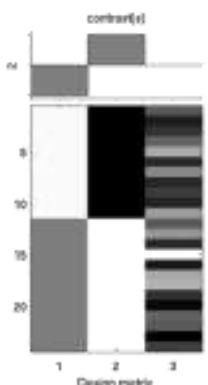

B
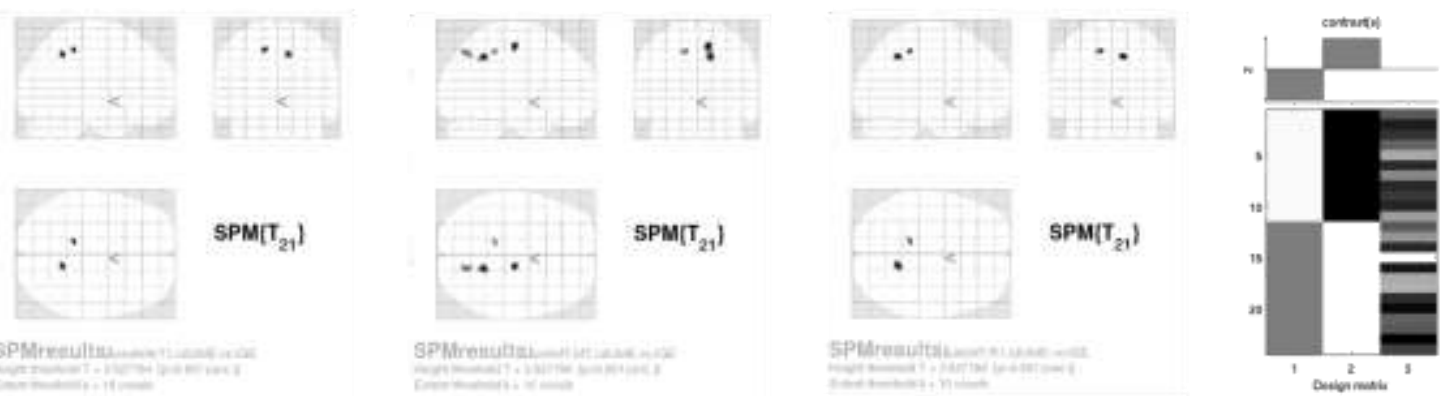

Abbildung 7: JME < IGE (A graue Substanz, B weiße Substanz)

A

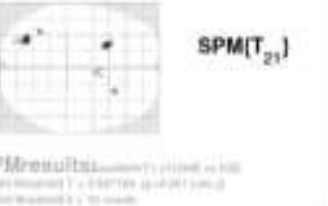

B

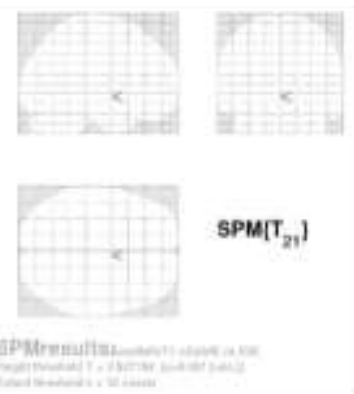

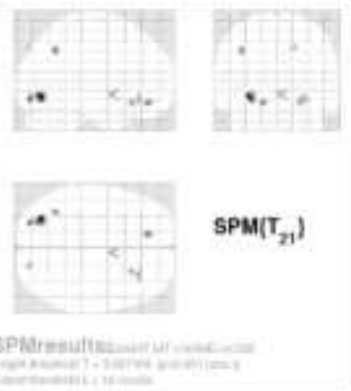
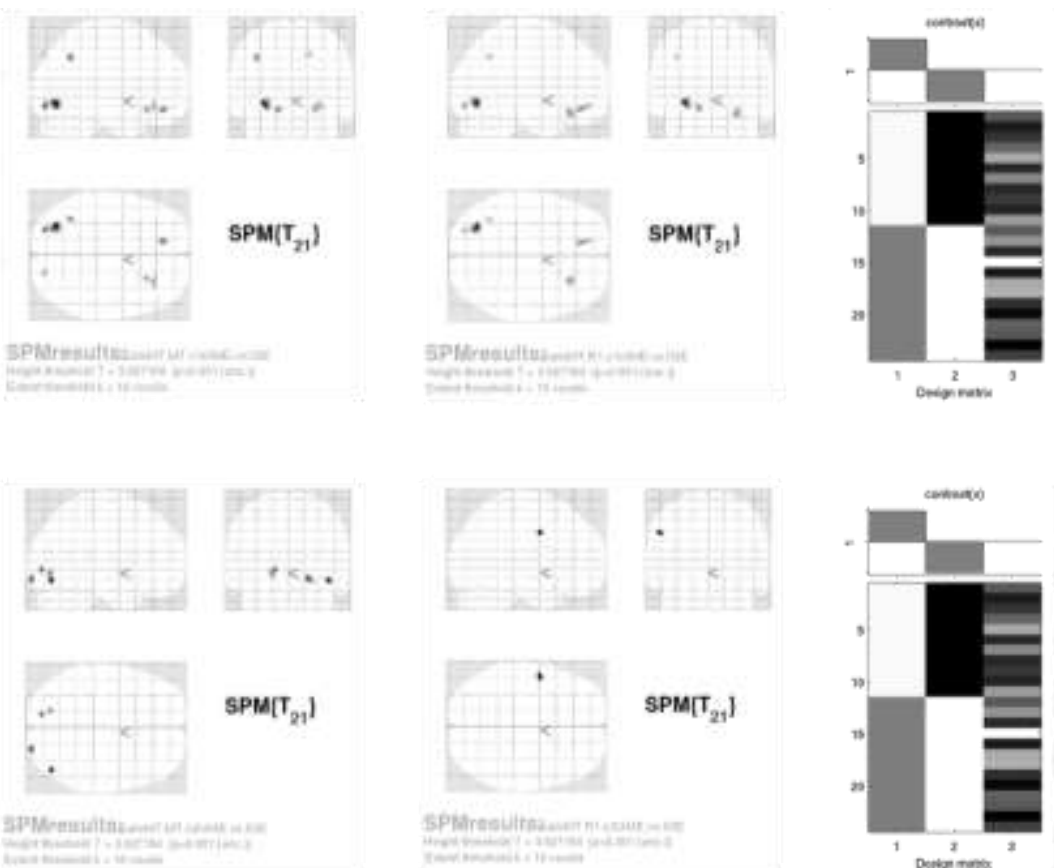
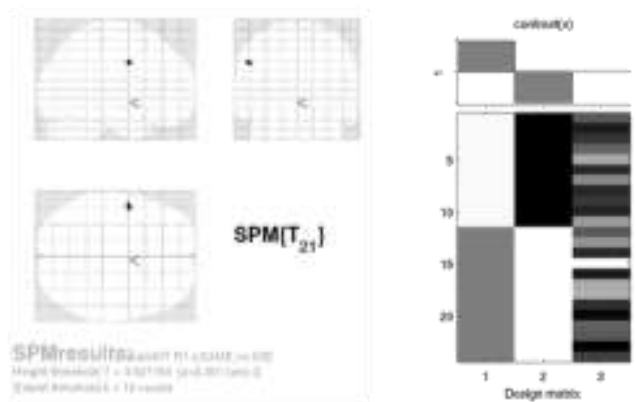

Abbildung 8: JME > IGE (A graue Substanz, B weiße Substanz) 


\subsubsection{Vergleich der DTI-Daten mit Tract-Based Spatial Statistics}

Die Analyse der DTI- Daten mit Tract- Based Spatial Statistics (TBSS) zeigte statistisch signifikant (korrigiert für multiple Tests und auf Cluster-Level mit Threshold-Free Cluster Enhancement, $\mathrm{p}<0,01$ ) eine Abnahme der fraktionellen Anisotropie (FA) sowie eine Zunahme des mittleren Diffusionskoeffizienten (mean diffusion, MD) innerhalb der weißen Substanz sowohl bei der JME- und der IGE-Gruppe als auch bei der zusammengefassten Patientengruppe (IGE und JME) im Vergleich zur Kontrollgruppe (vergleiche Abbildung 9). Eine Zunahme der FA oder ein Verlust des MD waren nicht signifikant. Ebenfalls fanden sich keine signifikanten Unterschiede der FA oder des MD im Vergleich beider Patientengruppen (IGE und JME) untereinander.

Die Cluster wurden mit Hilfe des JHU White-Matter Tractography Atlas sowie des JHU ICBM-DTI-81 White-Matter Labels den Arealen innerhalb der weißen Substanz zugeordnet.

Erläuterungen zu den folgenden Tabellen:

Cluster_Ind $=$ Clusterindex, $\mathrm{p}=\mathrm{p}$-Wert, $\mathrm{k}=$ Clustergröße in Voxel, $\mathrm{MNI}=$ Koordinaten auf der $\mathrm{x}$-, y- sowie z-Achse (orientierend am Standardgehirn des Montreal Neurological Instituts) 


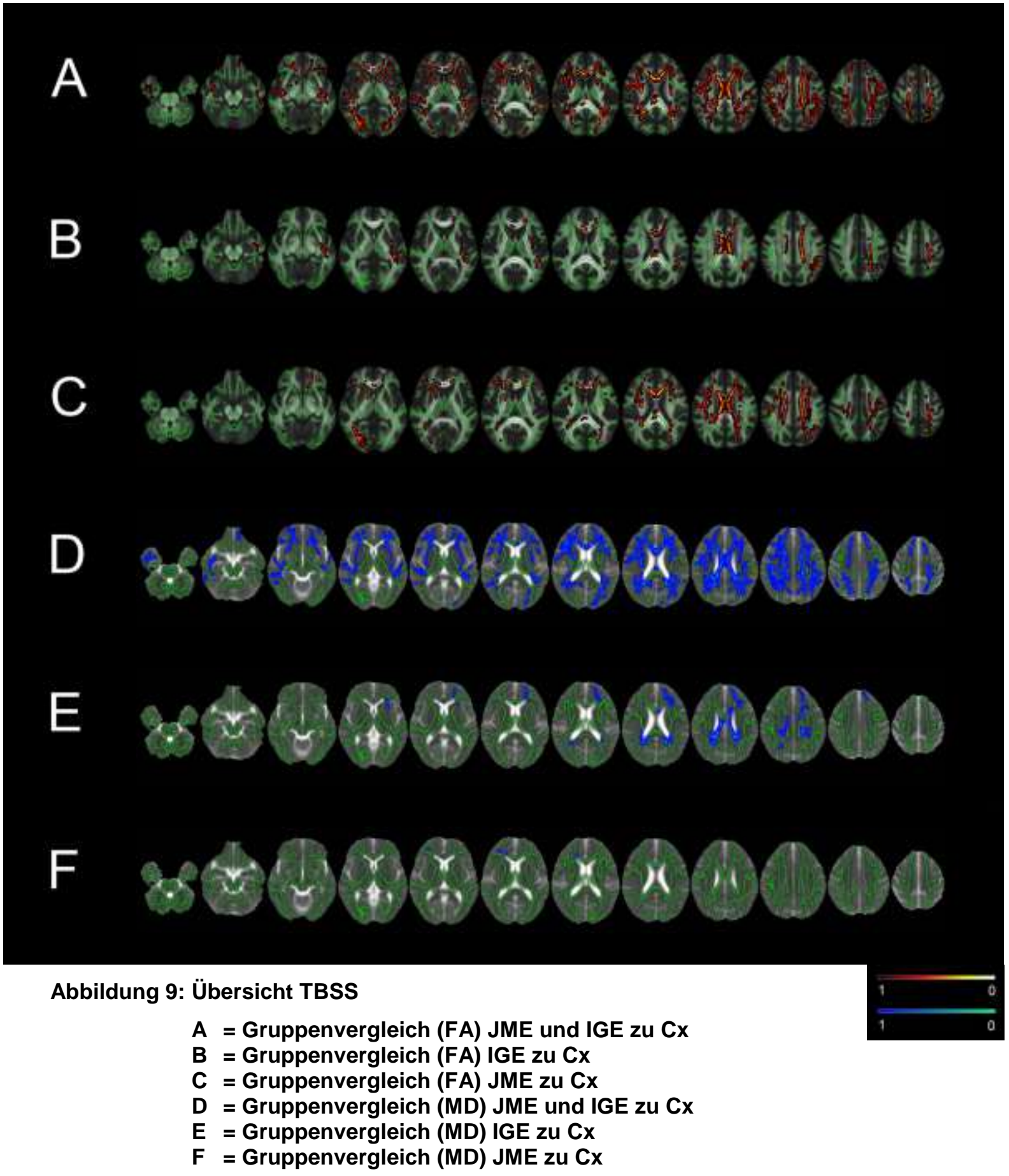

Informationen zu Abbildung 9: Die Abbildung zeigt die detektierten Unterschiede, wobei die farblichen Veränderungen für Ab- (rot) oder Zunahmen (blau) der FA bzw. des MD stehen. Zur besseren Darstellung der signifikanten Unterschiede wurde „tbss_fill“ verwendet. Der Farbbereich spiegelt den korrigierten $\mathrm{p}$-Wert wider.

\subsubsection{Vergleich beider Patientengruppen mit der Kontrollgruppe (FA)}

Der Vergleich beider Patientengruppen (JME und IGE) mit der Kontrollgruppe wies statistisch signifikant eine Abnahme der fraktionellen Anisotropie in 22,3\% des gesamten 
TBSS-Skeletts auf (korrigiert, $p<0,01$, vergleiche Tabelle 20). Diese Reduktionen fanden sich innerhalb eines großen Clusters mit 32076 Voxel und p-Werten von bis zu 0,001 bzw. 0,002 im Bereich des Truncus des Corpus callosum (MNI 3 -27 23, -15 -28 30, -6 -13 26, 3 -14 25, -7 -24 25), des linken Tractus corticospinalis (MNI -19 -22 37), des Fasciculus longitudinalis superior (MNI -21 -4738), der linken supplementär-motorischen Rinde (MNI -6 -10 63, -11 -2 62) und der linken Corona radiata (MNI -19 -33 35). Darüber hinaus waren weite Teile des Frontal-, Parietal-, Temporal- und Okzipitallappens involviert und es konnten 339 Sub-Cluster detektiert werden.

\begin{tabular}{|c|c|c|ccc|}
\hline Cluster_Ind & $\mathbf{p}$ & $\mathbf{k}$ & & MNI & \\
\hline 1 & 0,001 & 32076 & 3 & -27 & 23 \\
1 & 0,001 & & -21 & -47 & 38 \\
1 & 0,001 & & -19 & -22 & 37 \\
1 & 0,001 & & -19 & -33 & 35 \\
1 & 0,001 & -15 & -28 & 30 \\
1 & 0,001 & 14 & -26 & 29 \\
1 & 0,001 & -6 & -13 & 26 \\
1 & 0,001 & 3 & -14 & 25 \\
1 & 0,001 & -7 & -24 & 25 \\
1 & 0,002 & -6 & -10 & 63 \\
1 & & -11 & -2 & 62 \\
\hline
\end{tabular}

Tabelle 20: Gruppenvergleich (FA) von IGE und JME mit Cx

\subsubsection{Vergleich der JME-Patienten mit der Kontrollgruppe (FA)}

Bei der Analyse der DTI-Daten konnten im Vergleich der JME-Patienten mit der Kontrollgruppe verschiedene Cluster nachgewiesen werden, die einen Verlust der fraktionellen Anisotropie aufzeigten.

Das größte Cluster $(\mathrm{k}=11507)$ mit $\mathrm{p}$-Werten von 0,001 bzw. von 0,002 fand sich im Truncus (MNI 8 -19 26, -15 9 30, 134 30, -14 -23 30, 1513 29, 11 -4 29, 10 -16 28, -9 -17 27) sowie im Splenium (MNI 16 -35 29) des Corpus callosum, im rechten Tractus corticospinalis (MNI 25 -21 34) und im rechten Fasciculus longitudinalis superior (MNI 23 -43 34, 26 -34 29). Ein weiteres größeres Cluster $(k=1521, p=0,008$ und $p=0,009)$ konnte im Bereich der rechten Corona radiata (MNI 2710 24, 230 34), des rechten Fasciculus fronto-occipitalis inferior (MNI 2927 14, 3732 11) und des rechten Fasciculus longitudinalis superior (MNI 30330 , $37226)$ detektiert werden.

Zusätzlich zeigten sich weitere kleinere Cluster (vergleiche Tabelle 21). 


\begin{tabular}{|c|c|c|c|c|c|}
\hline Cluster_Ind & $\mathbf{p}$ & $\mathbf{k}$ & \multicolumn{3}{|c|}{ MNI } \\
\hline 5 & 0,001 & \multirow[t]{12}{*}{11507} & 8 & -19 & 26 \\
\hline 5 & 0,002 & & 25 & -21 & 34 \\
\hline 5 & 0,002 & & 23 & -43 & 34 \\
\hline 5 & 0,002 & & -15 & 9 & 30 \\
\hline 5 & 0,002 & & 13 & 4 & 30 \\
\hline 5 & 0,002 & & -14 & -23 & 30 \\
\hline 5 & 0,002 & & 15 & 13 & 29 \\
\hline 5 & 0,002 & & 11 & -4 & 29 \\
\hline 5 & 0,002 & & 26 & -34 & 29 \\
\hline 5 & 0,002 & & 16 & -35 & 29 \\
\hline 5 & 0,002 & & 10 & -16 & 28 \\
\hline 5 & 0,002 & & -9 & -17 & 27 \\
\hline \multicolumn{6}{|c|}{ [...] plus 122 weitere Sub-Cluster } \\
\hline 4 & 0,008 & \multirow[t]{11}{*}{1521} & 27 & 10 & 24 \\
\hline 4 & 0,008 & & 25 & 12 & 34 \\
\hline 4 & 0,008 & & 30 & 3 & 30 \\
\hline 4 & 0,008 & & 29 & 22 & 27 \\
\hline 4 & 0,008 & & 27 & 29 & 22 \\
\hline 4 & 0,009 & & 23 & 0 & 34 \\
\hline 4 & 0,009 & & 37 & 2 & 26 \\
\hline 4 & 0,009 & & 29 & 27 & 14 \\
\hline 4 & 0,009 & & 30 & 37 & 12 \\
\hline 4 & 0,009 & & 37 & 32 & 11 \\
\hline 4 & 0,009 & & 33 & 48 & 7 \\
\hline \multicolumn{6}{|c|}{ [...] plus 10 weitere Sub-Cluster } \\
\hline 3 & 0,009 & \multirow[t]{5}{*}{253} & 32 & -1 & 17 \\
\hline 3 & 0,01 & & 28 & -9 & 26 \\
\hline 3 & 0,01 & & 35 & -3 & 22 \\
\hline 3 & 0,01 & & 27 & -2 & 20 \\
\hline 3 & 0,01 & & 33 & 4 & 18 \\
\hline 2 & 0,009 & \multirow[t]{4}{*}{95} & 19 & 33 & 27 \\
\hline 2 & 0,01 & & 19 & 24 & 32 \\
\hline 2 & 0,01 & & 21 & 34 & 27 \\
\hline 2 & 0,01 & & 22 & 40 & 20 \\
\hline 1 & 0,01 & \multirow[t]{2}{*}{19} & 18 & 47 & 20 \\
\hline 1 & 0,01 & & 18 & 45 & 21 \\
\hline
\end{tabular}

Tabelle 21: Gruppenvergleich (FA) von JME mit Cx

\subsubsection{Vergleich der IGE-Patienten mit der Kontrollgruppe (FA)}

Die IGE-Patienten wiesen eine Abnahme der fraktionellen Anisotropie im Vergleich mit der Kontrollgruppe in verschiedenen Bereichen der weißen Substanz auf ( $p<0,01$, korrigiert). 
Das größte Cluser $(k=6368, p=0,003$ bzw. $p=0,004)$ fand sich hier im Truncus des Corpus callosum (MNI -17 -26 32, -17 -12 36, -15 0 34, -11 -15 30, -14 -26 29, 11 -18 28), im linken Fasciculus Iongitudinalis superior ( $\mathrm{MNI}-22$-45 38) sowie im linken Tractus corticospinalis (MNI -20 -34 53, -20 -25 48, -19 -22 37), in der linken Corona radiata (MNI -17 1235 ) und im linken Cingulum (MNI -18 -35 35). Weitere kleinere Cluster sind der Tabelle 22 zu entnehmen. Insgesamt finden sich die Reduktionen der FA vor allem auf der linken Seite.

\begin{tabular}{|c|c|c|ccc|}
\hline Cluster_Ind & $\mathbf{p}$ & $\mathbf{k}$ & & MNI & \\
\hline 7 & 0,003 & 6368 & -17 & -26 & 32 \\
7 & 0,004 & & -20 & -34 & 53 \\
7 & 0,004 & & -20 & -25 & 48 \\
7 & 0,004 & & -22 & -45 & 38 \\
7 & 0,004 & & -19 & -22 & 37 \\
7 & 0,004 & & -17 & -12 & 36 \\
7 & 0,004 & & -17 & 12 & 35 \\
7 & 0,004 & & -18 & -35 & 35 \\
7 & 0,004 & & -15 & 0 & 34 \\
7 & 0,004 & & -11 & -15 & 30 \\
7 & 0,004 & & -14 & -26 & 29 \\
7 & 0,004 & & 11 & -18 & 28 \\
\hline$\ldots]$ plus 58 weitere Sub-Cluster & & & & -34 \\
\hline 6 & 0,009 & 43 & -29 & -29 \\
\hline 6 & 0,01 & & -35 & -1 & 35 \\
\hline 5 & 0,009 & 35 & -31 & -64 & 38 \\
\hline 5 & 0,01 & & -30 & -62 & 31 \\
\hline 5 & 0,01 & & -34 & -68 & 16 \\
\hline 4 & 0,01 & 16 & -30 & -83 & 12 \\
\hline 3 & 0,01 & 10 & -29 & -77 & 26 \\
\hline 2 & 0,01 & 9 & -33 & -79 & -67 \\
\hline 1 & 0,01 & 2 & -34 & & \\
\hline
\end{tabular}

Tabelle 22: Gruppenvergleich (FA) von IGE mit Cx

\subsubsection{Vergleich der IGE- mit den JME-Patienten (FA)}

Die Untersuchung der DTI-Daten auf Unterschiede der FA zwischen beiden Patientengruppen erbrachte sowohl mit strenger Fehlerkorrektur für multiple Tests $(p<0,05)$ als auch bei liberaler Testung $(p<0,001)$ keine signifikanten Ergebnisse. 


\subsubsection{Vergleich beider Patientengruppen mit der Kontrollgruppe (MD)}

Die Analyse zeigte eine Zunahme des mittleren Diffusionskoeffizienten im Vergleich beider Patientengruppen mit der Kontrollgruppe in einem großen Cluster, das sich mit 25621 Voxel über weite Teile der weißen Substanz erstreckt (korrigiert, $p<0,01$, vergleiche Abbildung 9). Neben weiteren 333 Sub-Clustern fand sich ein Zunahme des MD im Besonderen in der rechten Forceps minor (MNI 1835 8), im linken Tractus corticospinalis (MNI -15 -36 56, -22 -35 52), im rechten Fasciculus longitudinalis superior (MNI 19 -46 56, 27 -49 56, 16 -64 51) sowie im rechten Cingulum (MNI $17-54$ 51).

\begin{tabular}{|c|c|c|rrr|}
\hline Cluster_Ind & $\mathbf{p}$ & $\mathbf{k}$ & & MNI & \\
\hline 1 & 0,001 & 25621 & 18 & 35 & 8 \\
1 & 0,002 & & -6 & 17 & 60 \\
1 & 0,002 & & -12 & 8 & 58 \\
1 & 0,002 & 15 & -8 & 56 \\
1 & 0,002 & -15 & -36 & 56 \\
1 & 0,002 & 19 & -46 & 56 \\
1 & 0,002 & 27 & -49 & 56 \\
1 & 0,002 & -8 & 25 & 54 \\
1 & 0,002 & 16 & 2 & 54 \\
1 & 0,002 & -22 & -35 & 52 \\
1 & & 17 & -54 & 51 \\
1 & & 16 & -64 & 51 \\
\hline
\end{tabular}

Tabelle 23: Gruppenvergleich (MD) von IGE und JME mit Cx

\subsubsection{Vergleich der JME-Patienten mit der Kontrollgruppe (MD)}

Die Analyse der Daten wies im Vergleich der JME-Patienten mit der Kontrollgruppe eine Zunahme des mittleren Diffusionskoeffizienten (korrigiert, $p<0,01$ ) vor allem in der Forceps minor (MNI 1732 15), in der rechten Corona radiata thalami (MNI 2938 13, 3240 9) sowie im rechten Fasciculus fronto-occipitalis inferior (MNI 31427 ) auf.

\begin{tabular}{|c|c|c|ccc|}
\hline Cluster_Ind & $\mathbf{p}$ & $\mathbf{k}$ & \multicolumn{3}{c|}{ MNI } \\
\hline 4 & 0,009 & 34 & 17 & 32 & 15 \\
\hline 3 & 0,009 & 7 & 29 & 38 & 13 \\
\hline 2 & 0,01 & 2 & 31 & 42 & 7 \\
\hline 1 & 0,01 & 1 & 32 & 40 & 9 \\
\hline
\end{tabular}

Tabelle 24: Gruppenvergleich (MD) von JME mit Cx 


\subsubsection{Vergleich der IGE-Patienten mit der Kontrollgruppe (MD)}

Bei den IGE-Patienten zeigten sich im Vergleich zu der Kontrollgruppe mehrere Bereiche in der weißen Substanz, die eine Zunahme des mittleren Diffusionskoeffizienten aufwiesen (korrigiert, $p<0,01$ ).

Das größte Cluster fand sich im Bereich des Truncus des Corpus callosum (MNI 12 -18 29, 13 -10 31, 3 -14 25), des rechten Tractus corticospinalis (MNI $24-27$ 30), des rechten Fasciculus longitudinalis superior (27 -61 29), und des rechten Fasciculus fronto-occipitalis inferior (MNI 27 -30 22, 28 -41 22). Weitere größere Cluster $(\mathrm{k}=713, \mathrm{k}=607)$ konnten ebenfalls im Truncus des Corpus callosum, der Forceps minor (MNI -12 131, -11 47 33, -18 41 6, -20 33 12, -9 -12 28, -10 -24 27, -15 46 23, -21 36 21, -18 48 12), im linken Cingulum (MNI -17 -29 32), im linken Fasciculus longitudinalis superior und inferior (MNI -28 -36 31, 29 -37 22, -24 -46 23) sowie im linken Fasciculus fronto-occipitalis inferior (MNI -13 35 37, 1736 30) detektiert werden. Weitere kleinere Cluster finden sich in Tabelle 25.

\begin{tabular}{|c|c|c|c|c|c|}
\hline Cluster_Ind & $p$ & k & & MNI & \\
\hline 16 & 0,007 & \multirow[t]{12}{*}{867} & 21 & -42 & 27 \\
\hline 16 & 0,008 & & 24 & -27 & 30 \\
\hline 16 & 0,008 & & 16 & -33 & 28 \\
\hline 16 & 0,008 & & 24 & -37 & 28 \\
\hline 16 & 0,009 & & 12 & -18 & 29 \\
\hline 16 & 0,009 & & 22 & -46 & 27 \\
\hline 16 & 0,01 & & 13 & -10 & 31 \\
\hline 16 & 0,01 & & 27 & -61 & 29 \\
\hline 16 & 0,01 & & 18 & -53 & 26 \\
\hline 16 & 0,01 & & 3 & -14 & 25 \\
\hline 16 & 0,01 & & 27 & -30 & 22 \\
\hline 16 & 0,01 & & 28 & -41 & 22 \\
\hline \multicolumn{6}{|c|}{ [...] plus 2 weitere Sub-Cluster } \\
\hline 15 & 0,007 & \multirow[t]{12}{*}{713} & -23 & -41 & 26 \\
\hline 15 & 0,008 & & -17 & -29 & 32 \\
\hline 15 & 0,008 & & -18 & -40 & 27 \\
\hline 15 & 0,009 & & -24 & -46 & 23 \\
\hline 15 & 0,009 & & -29 & -37 & 22 \\
\hline 15 & 0,009 & & -18 & -52 & 22 \\
\hline 15 & 0,01 & & -12 & 1 & 31 \\
\hline 15 & 0,01 & & -28 & -36 & 31 \\
\hline 15 & 0,01 & & -26 & -23 & 30 \\
\hline 15 & 0,01 & & -9 & -12 & 28 \\
\hline 15 & 0,01 & & -10 & -24 & 27 \\
\hline 15 & 0,01 & & -30 & -48 & 16 \\
\hline
\end{tabular}

Tabelle 25: Gruppenvergleich (MD) von IGE mit Cx 


\begin{tabular}{|c|c|c|ccc|}
\hline Cluster_Ind & $\mathbf{p}$ & $\mathbf{k}$ & & MNI & \\
\hline 14 & 0,009 & 607 & -11 & 47 & 33 \\
14 & 0,01 & & -8 & 42 & 41 \\
14 & 0,01 & & -16 & 27 & 40 \\
14 & 0,01 & & -13 & 35 & 37 \\
14 & 0,01 & & -17 & 36 & 30 \\
14 & 0,01 & & -15 & 46 & 23 \\
14 & 0,01 & & -21 & 36 & 21 \\
14 & 0,01 & & -18 & 48 & 12 \\
14 & 0,01 & & -20 & 33 & 12 \\
\hline 14 & 0,01 & & -18 & 41 & 6 \\
\hline 13 & 0,01 & 132 & -26 & 28 & 14 \\
\hline 12 & 0,01 & 103 & -27 & 21 & 23 \\
\hline 11 & 0,01 & 47 & -21 & 26 & -4 \\
\hline 10 & 0,01 & 31 & -4 & 7 & 23 \\
\hline 9 & 0,01 & 29 & -11 & 12 & 1 \\
\hline 8 & 0,01 & 24 & -26 & 20 & 33 \\
\hline 7 & 0,01 & 10 & -17 & 16 & 34 \\
\hline 6 & 0,01 & 8 & -18 & 27 & 34 \\
\hline 5 & 0,01 & 8 & -16 & 10 & 33 \\
\hline 4 & 0,01 & 8 & -24 & -27 & 13 \\
\hline 3 & 0,01 & 8 & -29 & -64 & 25 \\
\hline 2 & 0,01 & 3 & 11 & -18 & 32 \\
\hline 1 & 0,01 & 3 & & & 27 \\
\hline
\end{tabular}

Tabelle 25: Gruppenvergleich (MD) von IGE mit Cx, fortgesetzt

\subsubsection{Vergleich der IGE- mit den JME-Patienten (MD)}

Die TBSS-Analyse hinsichtlich Differenzen des MD zwischen beiden Patientengruppen erbrachte bei strenger Fehlerkorrektur keine signifikanten Daten $(p<0,05)$. Auch ohne Korrektur für multiple Tests zeigten sich keine signifikanten Unterschiede $(p<0,001)$. 


\section{Diskussion}

Ziel dieser Arbeit ist es, einen Beitrag zum Verständnis von idiopathisch-generalisierten Epilepsien unter besonderer Berücksichtigung des Janz-Syndroms zu leisten.

Bei dieser Form der Epilepsie wird von einer genetischen Disposition ausgegangen, ohne dass sich spezielle Alterationen im Routine-MRT finden lassen (vergleiche Kapitel 1). Einige Studien konnten in der Vergangenheit jedoch strukturelle Veränderungen bei verschiedenen IGE-Patienten nachweisen, wobei sich die Ergebnisse nicht eindeutig bzw. teilweise kontrovers zeigten (Huang et al. 2011; O'Muircheartaigh et al. 2011; Bernhardt et al. 2009; Betting et al. 2006; Woermann et al. 1999). Untersuchungen von DTI-Daten wiesen hingegen auf einheitlichere Alterationen hin (Liu et al. 2011; Vulliemoz et al. 2011) und werfen damit die Frage auf, ob Veränderungen innerhalb der weißen Substanz bzw. innerhalb zerebraler Netzwerke bei IGE-Patienten eine größere Rolle spielen als bisher angenommen.

Daher wurden im Rahmen dieser Arbeit potentielle Veränderungen zerebraler Netzwerke anhand von DTI-Daten untersucht und Unterschiede der fraktionellen Anisotropie sowie des mittleren Diffusionskoeffizienten mittels TBSS analysiert. Im Fokus standen weiter strukturelle Alterationen der weißen bzw. der grauen Substanz, welche mittels VBM detektiert werden sollten. Hierbei wurden verschiedene MRT-Sequenzen/-Karten verwendet (T1-gewichtet, MT-Karte, T1-Relaxometrie), um zusätzlich Hinweise auf eine Optimierung der MRT-Diagnostik aufzuzeigen. Sowohl in der Untersuchung mit TBSS als auch mit VBM fand jeweils eine Gegenüberstellung der einzelnen Patientengruppen (JME, IGE) sowie der kombinierten Patientengruppe (IGE und JME) mit der Kontrollgruppe statt. Ebenfalls sollten mögliche Unterschiede zwischen den beiden Patientengruppen in einem direkten Vergleich analysiert werden.

Die Untersuchung der DTI-Daten zeigte dabei signifikante ausgedehnte Reduktionen der FA bzw. Zunahmen des MD bei Patienten mit idiopathisch-generalisierter Epilepsie sowohl im Vergleich der kombinierten als auch im Vergleich der beiden einzelnen Patientengruppen (IGE, JME) zur Kontrollgruppe, welche in diesem Ausmaß bisher erstmalig detektiert werden konnten. Die Voxel-basierte Morphometrie der weißen und der grauen Substanz wies hingegen geringe Unterschiede auf, die nur ein unkorrigiertes Signifikanzniveau erreichten. Unter Verwendung einer strengen Fehlerkorrektur für multiple Tests mittels family wise error 
rate fanden sich keine signifikanten Ergebnisse für $p<0,05$. Dabei waren die Unterschiede der VBM, basierend auf den verschiedenen Sequenzen bzw. quantitativen Karten, ebenfalls nur marginal. Auch zwischen beiden Patientengruppen fanden sich im direkten Vergleich sowohl in der VBM als auch in der Analyse mit TBSS bei strenger Fehlerkorrektur keine signifikanten Werte. Bei liberalen Analysen ohne Fehlerkorrektur für multiple Tests zeigten sich in der VBM dezente Veränderungen $(p<0,001)$, welche unter anderem den prä- und postzentralen Gyrus sowie den Gyrus fusiformis betrafen. Die TBSS-Analyse blieb auch unkorrigiert für $p<0,001$ ohne signifikante Ergebnisse.

\subsection{Veränderungen zerebraler Netzwerke}

Die DTI-Analyse mit TBSS ergab eine signifikant verminderte fraktionelle Anisotropie in weiten Bereichen innerhalb der weißen Substanz der kombinierten Patientengruppe (IGE und JME) verglichen mit der Kontrollgruppe. Diese erstreckte sich über 22,3\% des gesamten TBSS-Skeletts und betraf vor allem das Corpus callosum, die weiße Substanz im Bereich der supplementär-motorischen Rinde, kortikospinale Trakte und den Fasciculus longitudinalis superior sowie inferior. Zudem zeigten sich Veränderungen im Frontal-, Temporal-, Parietalund Okzipitallappen. Diese Alterationen innerhalb der weißen Substanz konnten hier erstmalig in diesem Ausmaß gezeigt werden. Die Reduktion der FA spricht dabei für eine weniger gerichtete Diffusion von Wassermolekülen innerhalb der detektierten Cluster, was eine Veränderung der Integrität der Myelinscheiden bzw. der Axone in den entsprechenden Netzwerken vermuten lässt (vergleiche Kapitel 2.1.3).

Das Hauptcluster (32076 Voxel) erstreckte sich dabei unter anderem über große Teile des Corpus callosum. Dies lässt eine Schädigung der Kommissurenfasern annehmen, die die supplementär-motorische Rinde, den prämotorischen Kortex sowie den Motorkortex beider Hemisphären miteinander verbinden. Ähnliche Beobachtungen waren bisher sowohl mit Traktographie (Liu et al. 2011; Vulliemoz et al. 2011) als auch mit TBSS (O'Muircheartaigh et al. 2011) nur bei Patienten mit JME beschrieben worden. Zurückliegende Untersuchungen bei IGE-Patienten wiesen hingegen Differenzen im Zerebellum (Li et al. 2010) bzw. keine signifikanten Veränderungen (Liu et al. 2011) auf. Studien von generalisierten Epilepsien in Tiermodellen zeigten jedoch ebenfalls eine verminderte FA im Corpus callosum sowie in weiteren Trakten (Jones et al. 2011) und wurden mit dem Auftreten und der Schwere von generalisierten Anfällen im Tiermodell in Verbindung gebracht (Chahboune et al. 2009). Die in dieser Arbeit vorgestellten Ergebnisse sind damit sehr gut vereinbar, da die FA im Corpus callosum sowohl in der zusammengefassten Patientengruppe als auch in beiden einzelnen 
Gruppen vermindert war. Diese Veränderungen könnten sich damit zukünftig als Indikator für die Häufigkeit und Schwere von generalisierten epileptischen Anfällen erweisen.

Neben den Bereichen im Corpus callosum zeigte sich weiter eine reduzierte FA in der linken Corona radiata, in der unter anderem verschiedene thalamokortikale Projektionsbahnen verlaufen. Die Einbeziehung thalamokortikaler Fasern konnte diesbezüglich bereits bei Untersuchungen mit JME-Patienten gezeigt werden. Dabei fand sich mittels Traktographie (Liu et al. 2011) sowie mittels voxelweisem Vergleich der FA (Deppe et al. 2008) eine Reduktion beidseits im Crus anterius der Capsula interna sowie in weiteren thalamokortikalen Netzwerken (Keller et al. 2011; Deppe et al. 2008). Diese Beobachtung von DTI-Daten bei IGE-Patienten ist jedoch neu und könnte die Bedeutung dieser Netzwerke bei idiopathisch-generalisierten Epilepsien insgesamt weiter unterstreichen. Die Unterschiede zu vorausgegangenen Studien mit IGE-Patienten könnten hier durch die relativ hohe Anzahl nicht anfallsfreier Patienten in dieser Arbeit begründet sein (13/25), was möglicherweise einen Bezug zur Intensität der Erkrankung andeutet. Eine weitere mögliche Erklärung hierfür stellt ein kleineres Patientenkollektiv von zehn IGE-Patienten (Liu et al 2011) bzw. ein jüngeres Patientenkollektiv mit einem durchschnittlichen Alter von 26 Jahren ( $\mathrm{Li}$ et al. 2010) dar. Dabei erstreckte sich die Altersspanne in der Studie von Li et al. nur von 19 bis 36 Jahre, wodurch eine niedrigere Anzahl von Patienten mit einer hohen Rate von stattgefundenen generalisierten Anfällen (lifetime seizures) angenommen werden kann. Diesbezüglich könnten daher weitere Studien mit größeren Patientenkollektiven sowie mit einer breiteren Altersspanne der Patienten interessant sein, um die hier vorgestellten Ergebnisse erweitern.

Bei dem Vergleich der Gruppe IGE mit der Kontrollgruppe zeigten sich ebenfalls signifikante Unterschiede. Die fraktionelle Anisotropie war neben dem Corpus callosum sowohl in kortikospinalen (linker Tractus corticospinalis), in kortikokortikalen (linker Fasciculus longitudinalis superior) Trakten sowie in einem Teil des limbischen Systems (linker Gyrus cinguli) reduziert. Diese Veränderungen mittels DTI konnten hier erstmalig detektiert werden, da die bisherigen Untersuchungen, wie oben erwähnt, keine (Liu et al. 2011) bzw. nur Veränderungen im Zerebellum (Li et al. 2010) aufwiesen.

Neben einer verminderten FA im Truncus des Corpus callosum zeigte sich im Vergleich der Gruppe JME mit der Kontrollgruppe ebenfalls eine verminderte fraktionelle Anisotropie im Splenium des Corpus callosum sowie in der rechten Corona radiata, dem rechten Fasciculus fronto-occipitalis inferior und dem rechten Fasciculus longitudinalis superior. Diese 
Ergebnisse fanden sich ebenfalls teilweise in den oben vorgestellten Untersuchungen anderer Forschungsgruppen (Keller et al. 2011, Liu et al. 2011, Deppe et al. 2008) wieder und weisen weiter auf eine Störung der Verbindungen zum Okzipitalpol hin. Die verminderte FA im Splenium spricht dabei vor allem für eine Beeinträchtigung der verbindenden Fasern der Okzipitalpole beider Hemisphären, die verminderte FA im Fasciculus longitudinalis superior und inferior eher für eine Störung von Assoziationsfasern vom Frontal- bzw. Temporal- zum Okzipitallappen.

Die Untersuchung des mittleren Diffusionskoeffizienten stützt die oben dargestellten Ergebnisse. Bei der Analyse der DTI-Daten beider Patientengruppen (IGE und JME) mit der Kontrollgruppe fand sich ebenfalls eine Zunahme des MD in weiten Bereichen der weißen Substanz (25621 Voxel). Hierzu zählen der linke Tractus corticospinalis, der rechte Fasciculus longitudinalis superior sowie der rechte Gyrus cinguli. Diese Areale konnten bereits in der Analyse der FA bei der Gruppe JME (Fasciculus longitudinalis superior) sowie in der Gruppe IGE (Tractus corticospinalis), jedoch zum Teil in der anderen Hemisphäre (linker Gyrus cinguli) detektiert werden. Im Einzelgruppenvergleich des MD zeigte sich ebenfalls eine unterschiedliche Akzentuierung der Hemisphären. In der Gruppe IGE manifestierten sich die Veränderungen eher in der linken, in der JME-Gruppe vermehrt auf der rechten Seite. Dies scheint insgesamt die Betonung der linken bzw. rechten Hemisphäre je nach Patientengruppe bzw. Analysefokus zu revidieren, sodass diese wahrscheinlich als unspezifisch gewertet werden können.

Unterschiede im direkten Vergleich beider Patientengruppen miteinander zeigten keine signifikanten Veränderungen der FA oder des MD. Ein Gruppenunterschied konnte nicht dargestellt werden. Da die Untersuchung der kombinierten Patientengruppe (JME und IGE) noch stärkere Unterschiede aufwies als die Gegenüberstellungen der einzelnen Patientengruppen mit der Kontrollgruppe, scheinen die Auswirkungen einer größeren Patientengruppe gegenüber potentiellen spezifischen Syndromeffekten zu überwiegen.

\subsection{Strukturelle Alterationen in Verbindung mit Netzwerkveränderungen}

Die VBM der T1-gewichteten Sequenz, der MT-Karte sowie der T1-Relaxometrie zeigte keine strukturellen Veränderungen bei strengem korrigierten Signifikanzniveau $(p<0,05$, peak level und spatial extent FWE korrigiert). Die unkorrigierte VBM-Untersuchung der 
Gruppe IGE mit JME lässt jedoch eine Verminderung der grauen Substanz in der supplementär-motorischen Rinde des Motorkortex, im rechten und linken frontoorbitalen Kortex sowie im rechten und linken postzentralen Gyrus vermuten.

Der Verlust von grauer Substanz in diesen Arealen könnte eine Ergänzung der verminderten FA in den verbindenden Kommissurenfasern des Motorkortex sowie der supplementärmotorischen Rinde darstellen. Aufgrund der unkorrigierten Signifikanzschwelle können falsch positive Ergebnisse ( Fehler 1. Art) jedoch nicht ausgeschlossen werden.

Andere Studien wiesen Veränderungen in ähnlichen Bereichen, wie zum Beispiel im Frontallappen, auf. Diese Analysen gingen jedoch teilweise mit einer Zunahme der grauen Substanz (Lin et al. 2009; Wörmann et al. 1999), teilweise mit einem Verlust von grauer Substanz (Huang et al. 2011) einher. Eine Abnahme der grauen Substanz in den Thalami, wie sie in anderen Studien beschrieben werden (Huang et al. 2011; Lin et al. 2009; Kim et al. 2007), konnte hier nicht festgestellt werden. In den Arealen der Thalami zeigten sich jedoch Bereiche innerhalb der weißen Substanz, die einen Volumenverlust aufwiesen. Weitere Volumenabnahmen der weißen Substanz konnten in Arealen der supplementär-motorischen Rinde sowie im Gyrus postcentralis dargestellt werden, die die oben beschriebenen TBSSErgebnissen ergänzen könnten.

Hinweise für Volumenzunahmen fanden sich sowohl in der grauen als auch in der weißen Substanz nur sehr dezent und nicht signifikant bei korrigiertem Schwellenwert im Gyrus fusiformis sowie im Frontalpol beidseits.

Die einzelnen Gruppenvergleiche (IGE, JME) sowie die direkte Gegenüberstellung der Patientengruppen zeigten ebenfalls nur grenzwertige Veränderungen, die nicht die korrigierte Signifikanzschwelle erreichten. In den Untersuchungen mit der Kontrollgruppe und einer liberalen Fehlerkorrektur fanden sich eine Abnahme der grauen Substanz im Bereich des Gyrus fusiformis sowie im Gyrus postcentralis und eine Zunahme im Temporalpol. Der Vergleich der JME-Patienten mit der Kontrollgruppe als auch die direkte Untersuchung zwischen den beiden Patientengruppen wies auf einen stärkeren Verlust von weißer Substanz im Verhältnis zur Gruppe IGE hin. Insgesamt sind einige der unkorrigierten Ergebnisse gut mit anderen Studien sowie den Ergebnissen der TBSS-Untersuchung (siehe oben) vereinbar. Die fehlende korrigierte Signifikanz könnte dabei auf die eher kleinen Gruppengrößen sowohl bei der JME- (11 Patienten) als auch bei der IGE-Gruppe (13 Patienten) zurückzuführen sein. 


\subsection{Epileptogenese und strukturelle Auswirkungen}

Die strukturelle Analyse der T1-gewichteten Sequenz, der MT-Karte sowie der T1Relaxometrie erbrachte zwischen diesen Methoden zur MRT-Datenakquise keine wesentlichen Unterschiede. Obwohl die Untersuchung der DTI-Daten auf eine Störung der Integrität von Myelinscheiden hinweist und gerade die MT-Karte besonders zur Darstellung lipider Strukturen geeignet ist sowie einen guten Kontrast zwischen der weißen und grauen Substanz bietet (Helms et al. 2008), konnten auch hier keine signifikanten Veränderungen gezeigt werden. Dies könnte darauf hindeuten, dass die auch in anderen Studien detektierten, makrostrukturellen Veränderungen bei Epilepsie-Patienten im Vergleich zu Diffusionsstörungen eher geringer ausfallen bzw. sich die Veränderungen eher auf molekularer Ebene abspielen. Offen bleibt jedoch die Frage, ob diese Veränderungen primär eine Rolle in der Entstehung von epileptischen Anfällen darstellen oder ob sie sekundär durch diese hervorgerufen werden. Aktuell wird vermutet, dass bei idiopathischgeneralisierten Epilepsien eine Störung von lonenkanälen sowie Defekte bei Neurotransmitterrezeptoren vorliegen (Cossette et al. 2002). Da diese kleinsten Veränderungen keinen direkten Rückschluss auf Ab- bzw. Zunahmen von grauer und weißer Substanz erlauben, sprechen strukturelle Alterationen eher für einen sekundären Effekt durch epileptische Anfälle. Die in dieser Untersuchung detektierte Reduktion der FA bei beiden Patientengruppen (JME und IGE) würde damit die oben beschriebene Theorie untermauern und könnte einen Bezug zu generalisierten-tonisch-klonischen Anfällen darstellen.

Weiter zeigte die Studie von Deppe et al. (2008) eine negative Korrelation von FA und stattgefundenen Anfällen und unterstreicht damit ebenfalls die Theorie, dass eine reduzierte FA eine Folge von epileptischen Anfällen sein könnte. Die Untersuchungen von Vulliemoz et al. (2011) und Liu et al. (2011) widersprechen jedoch dieser Vermutung, da dort nur bei JMEPatienten eine signifikant verminderte FA gezeigt werden konnte und lassen damit eine endgültige Klärung von Auswirkung oder Ursache offen.

\subsection{Ausblick und Limitationen}

Insgesamt wird in dieser Studie deutlich, dass sich stärkere Veränderungen in den DTI- als in den volumetrischen Methoden - basierend auf T1- oder Multi-Parameter-Daten - fanden. Die Beteiligung der weißen Substanz in der Epileptogenese bzw. die Auswirkungen von epileptischen generalisierten Anfällen auf die Integrität von Netzwerken könnte durch diese Studie deutlicher in den Vordergrund rücken, da sich hier Veränderungen fast im gesamten 
TBSS-Skelett zeigten und das Ausmaß der Netzwerkalterationen bisher in dieser Form nicht dargestellt werden konnte. Diese Arbeit bestätigt damit die Beteiligung thalamokortikaler und kortikokortikaler Netzwerke, wobei sich interessanterweise ähnliche Veränderungen bei der Gruppe IGE sowie bei der Gruppe JME fanden. Da das hier detektierte Muster an FAVeränderungen auch in anderen Studien mit Tiermodellen (Jones et al. 2011) gezeigt wurde, könnte dies möglicherweise charakteristisch für IGE und damit eventuell zukünftig ein Marker für die Entwicklung sowie für den Verlauf diese Epilepsieform sein.

Die Magnetresonanztomographie bietet damit neben dem EEG sehr gute diagnostische Möglichkeiten, wobei weitere Studien bezüglich der Veränderungen von DTI-Daten von IGEPatienten interessant wären. Wünschenswert wären dabei ein größeres Patientenkollektiv, eine optimalere Einteilung der Patientengruppe zum Beispiel nach genetischer Testung und eine Erfassung der Patienten ab dem Zeitpunkt der Diagnosestellung, um Effekte durch antiepileptische Medikation zu minimieren bzw. aufklären zu können. Diese Faktoren stellen die Limitationen dieser Arbeit dar, da eine genetische Testung der involvierten Patienten nicht verfügbar war und sich Multizenter-Studien bisher als schwierig erwiesen haben (Focke et al. 2011). Studien mit langfristiger Begleitung von IGE-Patienten unter Berücksichtigung der oben genannten Punkte könnten sich hierbei als interessant erweisen und weitere Aspekte aufzeigen. 


\section{$5 \quad$ Zusammenfassung}

Epilepsie stellt mit einer Prävalenz von 0,45 - 0,7 \% (Forsgren et al. 2005) eine häufige und alle Altersklassen betreffende Erkrankung dar, die individuell zu einer enormen Einschränkung der Lebensqualität führen kann. Die Diagnose wird im Regelfall elektroklinisch, das heißt anhand der Klinik, des EEG-Befundes und gegebenenfalls mittels MRTBildgebung gestellt. Idiopathisch-generalisierte Epilepsien sind dabei durch eine genetische Prädisposition, primär generalisierte Anfälle sowie durch ein unauffälliges Routine-MRT gekennzeichnet. Klinische Studien konnten jedoch durch moderne Nachbearbeitungsverfahren von MRT-Daten (VBM) verschiedene zerebrale Alterationen bei IGE-Patienten aufzeigen, wobei diese teilweise widersprüchlich waren (O'Muircheartaigh et al. 2011; Betting et al. 2006; Woermann et al. 1999). Analysen von DTI-Daten wiesen hingegen in neueren Studien (Liu et al. 2011; Vulliemoz et al. 2011) auf deutlichere Veränderungen innerhalb zerebraler Trakte und somit auf mikrostrukturelle Alterationen hin, die durch VBM offensichtlich weniger erfasst werden. In dieser Studie sollten die genauen Veränderungen bei IGE sowie bei der gut klassifizierten Subgruppe „juvenile myoklonische Epilepsie“ anhand von VBM- und TBSS-Analysen aufgezeigt werden. Des Weiteren sollten verschiedene MRT-Sequenzen (T1, MT-Karte, T1-Relaxometrie) verwendet und somit deren Nutzen bezüglich einer möglichen Verbesserung der Epilepsie-Diagnostik herausgestellt werden.

In dieser Arbeit wurden dabei 25 Patienten (13 IGE und 12 JME) sowie 44 gesunde Kontrollprobanden im 3-Tesla-MRT der Universitätsmedizin Göttingen untersucht. Die Zuordnung zu der jeweiligen Epilepsieform basierte auf der Diagnose der behandelnden Ärzte aufgrund der Klinik und der technischen Untersuchungsbefunde (EEG, MRT). Neben der MRT-Untersuchung wurden weitere Informationen bezüglich des Alters, der Dauer der Erkrankung sowie der Anfallsfreiheit erfragt. Die aus den T1-gewichteten Bildern und die aus dem quantitativen Multi-Parameter Mapping gewonnenen Daten wurden mittels Voxelbasierter Morphometrie analysiert (13 IGE, 11 JME, 38 Kontrollprobanden), Daten aus der DTI-Sequenz anhand von TBSS (13 IGE, 12 JME, 44 Kontrollprobanden) untersucht und ausgewertet. Dabei wurden die beiden Patientengruppen einzeln und kombiniert mit der Kontrollgruppe verglichen sowie einander gegenübergestellt. Bei der VBM wurden Hinweise auf Volumenveränderungen der grauen sowie der weißen Substanz (unkorrigiert, $p<0,001$ ) detektiert, welche jedoch unter strenger Fehlerkorrektur für multiple Tests (FWE-Korrektur) das Signifikanzniveau $(p<0,05)$ nicht erreichten. 
Die TBSS-Analyse erbrachte signifikante Reduktionen der FA sowie Erhöhungen des MD in weiten Teilen zerebraler Trakte im Vergleich der kombinierten Patientengruppe mit der Kontrollgruppe $(22,3 \%$ des gesamten TBSS-Skeletts, korrigiert für multiple Tests, $p<0,01)$. Besonders betroffen war dabei das Corpus callosum, kortikospinale sowie thalamokortikale Trakte, Areale im Bereich des supplementär-motorischen Kortex sowie des Fasciculus longitudinalis superior und inferior. Im Einzelvergleich der IGE- bzw. JME-Gruppe fanden sich ebenfalls Cluster in diesen Bereichen, jedoch weniger ausgeprägt als in der kombinierten Patientengruppe. Ein signifikanter Unterschied zwischen der IGE- und JMEGruppe konnte nicht festgestellt werden.

Zwischen den T1-gewichteten Sequenzen sowie dem quantitativen Multi-Parameter Mapping fanden sich ebenfalls keine eindeutigen Unterschiede.

Die hier dargestellten Ergebnisse stellen die Veränderungen der weißen Substanz bzw. den Verlust der Integrität von Netzwerken in den Vordergrund, wobei hier aufgrund des Querschnitt-Designs der Studie keine endgültige Klärung bezüglich Ursache oder Folge generalisierter Anfälle möglich ist. Das detektierte Muster an FA-Reduktion bzw. MDErhöhung wurde teilweise bereits in Studien mit Tiermodellen beschrieben (Jones et al. 2011) und könnte sich eventuell als charakteristisch für idiopathisch-generalisierte Epilepsien erweisen. Darüber hinaus wurde dieses mit der Entstehung und Schwere von generalisierten Epilepsien in der Studie von Chahboune et al. 2009 in Verbindung gebracht und könnte damit zukünftig möglicherweise als Verlaufs- bzw. diagnostischer Marker dienen. Weitere longitudinale Studien mit Einbezug genetischer Testung und Beobachtung von möglichen pharmakotherapeutischen Auswirkungen auf diese Veränderungen wären deshalb interessant und nötig, um die bisherigen Ergebnisse weiter zu bestätigen und auszubauen. 


\section{$6 \quad$ Literaturverzeichnis}

Abou-Khalil B, Krei L, Lazenby B, Harris PA, Haines JL, Hedera P (2007): Familial genetic predisposition, epilepsy localization and antecedent febrile seizures. Epilepsy Res $\underline{73}$, 104-110

Alexander R (2013): Epilepsy. Handb Clin Neurol 116, 491-497

Ashburner J (2007): A fast diffeomorphic image registration algorithm. Neuroimage $\underline{38}(1)$, 95113

Ashburner J, Friston KJ (1997): Multimodal Image Coregistration and Partitioning- A Unified Framework. Neurolmage $\underline{6}, 209-217$

Ashburner J, Friston KJ (2000): Voxel-based Morphometry-The Methods. Neurolmage 11, 805-821

Austin JK, de Boer HM, Shafer PO: Disruptions in Social Functioning and Services Facilitaing Adjustment for the Child and the Adult; in Epilepsy: a comprehensive textbook, Volume III; hrsg. v. Engel J Jr, Pedley TA; Lippincott Williams \& Wilkins, Philadelphia 2008, 2237-2245

Banerjee P N, Filippi D, Allen Hauser W (2009): The descriptive epidemiology of epilepsy-a review. Epilepsy Res $\underline{85}$, 31-45

Basser PJ, Pierpaoli C (1996): Microstructural and Physiological Features of Tissues Elucidated by Quantitative-Diffusion-Tensor MRI. J Magn Reson, Series B 111, 209219

Berg AT, Berkovic SF, Brodie MJ, Buchhalter J, Cross JH, Boas W v E, Engel J, French J, Glauser TA, Mathern GW (2010): Revised terminology and concepts for organization of seizures and epilepsies: Report of the ILAE Commission on Classification and Terminology, 2005-2009. Epilepsia 51, 676-685

Berkovic SF, Mclntosh AM, Kalnins RM, Jackson GD, Fabinyi GC, Brazenor GA, Bladin PF, Hopper JL (1995): Preoperative MRI predicts outcome of temporal lobectomy: An actuarial analysis. Neurology $\underline{45}, 1358-1363$ 
Bernhardt BC, Rozen DA, Worsle KJ, Evans AC, Bernasconi N, Bernasconi A (2009): Thalamo-cortical network pathology in idiopathic generalized epilepsy: Insights from MRI-based morphometric correlation analysis. Neurolmage $\underline{46}$, 373-381

Betting LE, Mory SB, Li LM, Lopes-Cendes I, Guerreiro MM, Guerreiro CAM, Cendes F (2006): Voxel-based morphometry in patients with idiopathic generalized epilepsies. Neurolmage $\underline{32}, 498-502$

Blumenfeld H (2003): From Molecules to Networks: Cortical/Subcortical Interactions in the Pathophysiology of Idiopathic Generalized Epilepsy. Epilepsia 444, 7-15

Cercignani M, Bozzali M, Iannucci G, Comi G, Filippi A (2001): Magnetisation transfer ratio and mean diffusivity of normal appearing white and grey matter from patients with multiple sclerosis. J Neurol Neurosurg Psychiatry $\underline{70}$, 311-317

Chahboune H, Mishrab AM, DeSalvod MN, Staibc LH, Purcarod M, Scheinosta D, Papademetrisa X, Fysonf SJ, Lorinczf ML, Crunellif V (2009): DTI abnormalities in anterior corpus callosum of rats with spike-wave epilepsy. Neurolmage $\underline{47}, 459-466$

Chinchure S, Kesavadas C, Thomas B (2010): Structural and functional neuroimaging in intractable epilepsy. Neurol India $\underline{58}, 361-370$

Commission on Classification and Terminology of the International League Against Epilepsy (1981): Proposal for revised clinical and electroencephalographic classification of epileptic seizures. Epilepsia 22, 489-501

Cossette P, Liu L, Brisebois K, Dong H, Lortie A, Vanasse M, Saint-Hilaire JM, Carmant L, Verner A, Lu WY (2002): Mutation of GABRA1 in an autosomal dominant form of juvenile myoclonic epilepsy. Nat Genet $\underline{31}, 184-189$

Deoni SC (2010): Quantitative relaxometry of the brain. Top Magn Reson Imaging 21, 101113

Deppe M, Kellinghaus C, Duning T, Möddel G, Mohammadi S, Deppe K, Schiffbauer H, Kugel H, Keller SS, Ringelstein EB (2008): Nerve fiber impairment of anterior thalamocortical circuitry in juvenile myoclonic epilepsy. Neurology $\underline{71}, 1981-1985$

Deutsche Gesellschaft für Neurologie: S1-Leitlinie "Erster epileptischer Anfall und Epilepsien im Erwachsenenalter" (http://www.awmf.org/uploads/tx_szleitlinien/030-041I_S1_ Erster_epileptischer_Anfall_und_Epilepsien_im_Erwachsenenalter_2013-08_1.pdf) 
Dichter MA: Overview: The Neurobiology of Epilepsy; in: Epilepsy: a comprehensive textbook, Volume I; hrsg. v. Engel J Jr, Pedley TA; Lippincott Williams \& Wilkins, Philadelphia 2008, 216-218

Engel J Jr (2006): Report of the ILAE Classification Core Group. Epilepsia 4ㄱ, 1558-1568

Filippi M, Tortorella C, Bozzali M (1999): Normal-appearing white matter changes in multiple sclerosis: the contribution of magnetic resonance techniques. Mult Scler. $\underline{5}, 273-282$

Fisher R, Salanov V, Witt T, Worth R, Henry T, Gross R, Oommen K, Osorio I, Nazzaro J, Labar D, SANTE Study Group (2010): Electrical stimulation of the anterior nucleus of thalamus for treatment of refractory epilepsy. Epilepsia $\underline{51}, 899-908$

Fisher RS, Boas W v E, Blume W, Elger C, Genton P, Lee P, Engel J (2005): Epileptic Seizures and Epilepsy: Definitions Proposed by the International League Against Epilepsy (ILAE) and the International Bureau for Epilepsy (IBE). Epilepsia 46, 470472

Fisher RS, Acevedo C, Arzimanoglou A, Bogacz A, Cross JH, Elger C, Engel JJ Jr, French J, Glynn M, Hesdorffer D (2013): An Operational Clinical Definition of Epilepsy. Veröffentlicht auf der Homepage der Internationalen Liga gegen Epilepsie, http://www.ilae.org/Visitors/Centre/Definition.cfm, entnommen am 27.06.2014

Focke NK, Helms G, Kaspar S, Diederich C, Tóth V, Dechent P, Mohr A, Paulus W (2011): Multi-site voxel-based morphometry - not quite there yet. Neurolmage $\underline{56}, 1164-1170$

Forsgren L, Beghi E, Õun A, Sillanpää M (2005): The epidemiology of epilepsy in Europe - a systematic review. Eur J Neurol 12, 245-253

Fröscher W, Vassella F, Hufnagel A: Die Epilepsien. Grundlagen-Klinik-Behandlung. 2. Auflage. Schattauer $\mathrm{GmbH}$, Stuttgart 2004

Fusco L, Specchio N, Yagi K, Seino M, Vigevano F: Generalized Tonic Seizures; in: Epilepsy: a comprehensive textbook, Volume III; hrsg. v. Engel J Jr, Pedley TA; Lippincott Williams \& Wilkins, Philadelphia 2008, 610-618

Gringel T, Schulz-Schaeffer W, Elolf E, Frölich A, Dechent P, Helms G (2009): Optimized high-resolution mapping of magnetization transfer (MT) at 3 Tesla for direct visualization of substructures of the human thalamus in clinically feasible measurement time. J Magn Reson Imaging 29, 1285-1292 
Hacke W: Neurologie. 13. Auflage; Springer Medizin Verlag Heidelberg, Heidelberg 2010

Helms G, Dathe H, Kallenberg K, Dechent P (2008): High-Resolution Maps of Magnetization Transfer with Inherent Correction for RF Inhomogeneity and $T_{1}$ Relaxation Obtained from 3D FLASH MRI; Magn Reson Med 무, 1396-1407

Hobbs N, Novak M (2008): VBM-Voxel-based morphometry. Power-Point-Präsentation Seminarreihe (http://www.fil.ion.ucl.ac.uk/spm/doc/mfd/2007/MfDweb.htm)

Hsu WY, Cheng CH, Lin MW, Shih YH, Liao KK, Lin YY (2011): Antiepileptic effects of low frequency repetitive transcranial magnetic stimulation: A meta-analysis. Epilepsy Res. $\underline{96}(3), 231-40$

Huang W, Lu G, Zhang Z, Zhong Y, Wang Z, Yuan C, Jiao Q, Qian Z, Tan Q, Chen G, Zhang $Z$ (2011): Gray-matter volume reduction in the thalamus and frontal lobe in epileptic patients with generalized tonic-clonic seizures. J Neuroradiology $\underline{38}, 298-303$

ILAE Commission Report (1997): Recommendations for Neuroimaging of Patients with Epilepsy. Epilepsia 38, 1255-1256

Jackson GD, Kuzniecky RI: Structural Neuroimaging; in: Epilepsy: a comprehensive textbook, Volume III; hrsg. v. Engel J Jr, Pedley TA; Lippincott Williams \& Wilkins, Philadelphia 2008, 917-944

Jacoby A, Snape D, Baker GA: Social Aspects; in: Epilepsy: a comprehensive textbook, Volume III; hrsg. v. Engel J Jr, Pedley TA; Lippincott Williams \& Wilkins, Philadelphia 2008, 2228-2236

Jeha LE, Najm I, Bingaman W, Dinner D, Widdess-Walsh P, Lüders H (2007): Surgical outcome and prognostic factors of frontal lobe epilepsy surgery. Brain $\underline{130}, 574-584$

Johansen-Berg $\mathrm{H}$, Behrens TEJ: Diffusion MRI: from quantitative measurement to in-vivo neuroanatomy. 1. Auflage; Academic Print (Elsevier-Verlag), London 2009

Johnston D, Brown TH (1984): The synaptic nature of the paroxysmal depolarizing shift in hippocampal neurons. Ann Neurol 16, S65-71

Jones NC, O'Brien TJ, Powell KL (2011): Morphometric changes and molecular mechanismsin rat models of idiopathic generalized epilepsy with absence seizures. Neurosci Lett 497, 185-193 
Keller SS, Ahrens T, Mohammadi S, Möddel G, Kugel H, Ringelstein EB, Deppe M (2011): Microstructural and volumetric abnormalities of the putamen in juvenile myoclonic epilepsy. Epilepsia $\underline{52}, 1715-1724$

Kerrigan JF, Litt B, Fisher RS, Cranstoun S, French JA, Blum DE, Dichter M, Shetter A, Baltuch G, Jaggi J, Krone S (2004): Electrical Stimulation of the Anterior Nucleus of the Thalamus for the Treatment of Intractable Epilepsy. Epilepsia 45, 346-354

Kim JH, Lee JK, Koh SB, Lee SA, Lee JM, Kim SI, Kang JK (2007): Regional grey matter abnormalities in juvenile myoclonic epilepsy: a voxel-based morphometry study. Neurolmage $\underline{37}, 1132-1137$

Kwan P, Brodie MJ (2000): Early identification of refractory epilepsy. N Engl J Med 342, 314319

Lai V, Mak HK, Yung AW, Ho WY, Hung KN (2010): Neuroimaging techniques in epilepsy. Hong Kong Med J 16, 292-298

Le Bihan D, Mangin JF, Poupon C, Clark CA, Pappata S, Molko N, Chabriat H (2001): Diffusion tensor imaging: Concepts and applications. J Magn Reson Imaging 13, 534546

Li Y, Du H, Xie B, Wu N, Wang J, Wu G, Feng H, Jiang T (2010): Cerebellum abnormalities in idiopathic generalized epilepsy with generalized tonic-clonic seizures revealed by diffusion tensor imaging. PLoS One $\underline{5}$, e15219

Lin K, Jackowski AP, Carrete H Jr, de Araújo Filho GM, Silva HH, Guaranha MS, Guilhoto LM, Bressan RA, Yacubian EM (2009): Voxel-based morphometry evaluation of patients with photosensitive juvenile myoclonic epilepsy. Epilepsy Res $\underline{\text { 86, }}$, 138-145

Liu M, Concha L, Beaulieu C, Gross DW (2011): Distinct white matter abnormalities in different idiopathic generalized epilepsy syndromes. Epilepsia $\underline{52}, 2267-2275$

Loewenhardt B: Bildgebende Diagnostik: Technik/Anatomie/Pathologie. 3. Auflage; Wissenschaftlicher Selbstverlag, Fulda 2006

Masuhr KF, Masuhr F, Neumann M: Duale Reihe: Neurologie. 7. Auflage; Georg Thieme Verlag, Stuttgart 2013

Mattle H, Mumenthaler M: Neurologie. 13. Auflage; Georg Thieme Verlag, Stuttgart 2013 
Mattson, R (2003), Overview: Idiopathic Generalized Epilepsies. Epilepsia 44, 2-6

McCormick DA, Contreras D (2001): On the cellular and network bases of epileptic seizures. Annu Rev Physiol 63, 815-846

Mikati MA, Comair YG, Rahi A (2006): Normalization of quality of life three years after temporal lobectomy: a controlled study. Epilepsia $\underline{47}, 928-933$

O'Muircheartaigh J, Vollmar C, Barker GJ, Kumari V, Symms MR, Thompson P, Duncan JS, Koepp MJ, Richardson MP (2011): Focal structural changes and cognitive dysfunction in juvenile myoclonic epilepsy. Neurology $\underline{76}, 34-40$

Osorio I, Overman J, Giftakis J, Wilkinson SB (2007): High Frequency Thalamic Stimulation for Inoperable Mesial Temporal Epilepsy. Epilepsia 4으, 1561-1571

Pastor J, Ortega GJ, Herrera-Peco I, Navas-García M, Navarrete EG, Alonso-Cerezo C, Pulido-Rivas P, Sola RG (2010): Differential contribution of preoperatory studies to diagnosis in temporal lobe epilepsy surgery. Rev Neurol $\underline{51}$, 393-402

Reiser M, Semmler W: Magnetresonanztomographie, 3. Auflage; Springer-Verlag, Berlin Heidelberg New York 2002

Reiser M, Kuhn FP, Debus J: Duale Reihe: Radiologie, 3. Auflage; Georg Thieme Verlag, Stuttgart 2011

Rosenow F, Lüders H (2001): Presurgical evaluation of epilepsy. Brain 124, 1683-1700

Salmond CH, Ashburner J, Vargha-Khadem F, Connelly A, Gadian DJ, Friston KJ (2002):

Distributional Assumptions in Voxel-Based Morphometry. Neurolmage 17, 1027-1030

Sankar R, Rho JM: Neurophysiologic Basis of Epileptiform Activity. In: Advanced Therapy in Epilepsy; hrsg. v. Wheless JW, Willmore LJ, Brumback RA; People's Medical Publishing House, Shelton (USA) 2009, 16-22

Schulze-Bonhage A (2009): Tiefe Hirnstimulation als neuer Therapieansatz bei Epilepsien. Dtsch Arztebl 106, 407-412

Smith SM, Johansen-Berg H, Jenkinson M, Rueckert D, Nichols TE, Miller KL, Robson MD, Jones DK, Klein JC, Bartsch AJ (2007): Acquisition and voxelwise analysis of multisubject diffusion data with Tract-Based Spatial Statistics. Nat Protoc 2, $499-503$ 
Tettenborn B , Bredel-Geissler A-E, Krämer G: Die Epilepsie. In Berlit P (Hrsg.): Klinische Neurologie. 2. Auflage, Springer Medizin Verlag, Heidelberg 2006, 741-748

Vlaardingerbroek MT, den Boer JA: Magnetresonanzbildgebung: Theorie und Praxis; Springer, Berlin/Heidelberg 2004

Vulliemoz S, Vollmar C, Koepp MJ, Yogarajah M, O'Muircheartaigh J, Carmichael DW, Stretton J, Richardson MP, Symms MR, Duncan JS (2011): Connectivity of the supplementary motor area in juvenile myoclonic epilepsy and frontal lobe epilepsy. Epilepsia $\underline{52}, 507-514$

Wehner T, Lüders H (2008): Role of Neuroimaging in the Presurgical Evaluation of Epilepsy. $\mathrm{J}$ Clin Neurol $\underline{4}, 1-16$

Weishaupt D, Köchli VD, Marincek B: Wie funktioniert MRI? Eine Einführung in Physik und Funktionsweise der Magnetresonanzbildgebung. 6. Auflage; Springer Medizin Verlag, Heidelberg 2009

Wiebe S, Blume WT, Girvin JP, Eliasziw M, Effectiveness and Efficiency of Surgery for Temporal Lobe Epilepsy Study Group, (2001): A Randomized, Controlled Trial of Surgery for Temporal-Lobe Epilepsy. N Engl J Med $\underline{345}, 311-318$

Woermann FG, Free SL, Koepp MJ, Sisodiya SM, Duncan JS (1999): Abnormal cerebral structure in juvenile myoclonic epilepsy demonstrated with voxel-based analysis of MRI. Brain 122, 2101-2108

Wojtecki L, Südmeyer M, Schnitzler A (2007): Therapie des ideopathischen ParkinsonSyndroms. Dtsch Arztebl 104, 2513-2522

Wolff SD, Balaban RS (1989): Magnetization transfer contrast (MTC) and tissue water proton relaxation in vivo. Magn Reson Med $\underline{10}, 135-144$ 


\section{$7 \quad$ Abbildungsverzeichnis}

Abbildung 1: JME und IGE < Cx ( A graue Substanz, B weiße Substanz) ..........................27

Abbildung 2: JME und IGE > Cx (A graue Substanz, B weiße Substanz) ..........................27

Abbildung 3: IGE < Cx (A graue Substanz, B weiße Substanz) ......................................30

Abbildung 4: IGE > Cx (A graue Substanz, B weiße Substanz) .....................................30

Abbildung 5: JME < Cx (A graue Substanz, B weiße Substanz) .......................................34

Abbildung 6: JME > Cx (A graue Substanz, B weiße Substanz) .....................................34

Abbildung 7: JME < IGE (A graue Substanz, B weiße Substanz) .....................................37

Abbildung 8: JME > IGE (A graue Substanz, B weiße Substanz) ....................................37

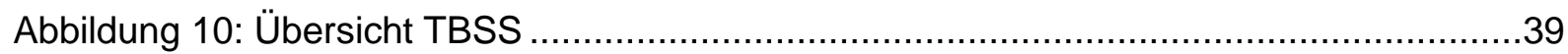




\section{Tabellenverzeichnis}

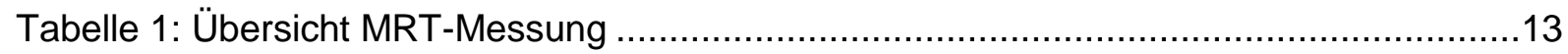

Tabelle 2: Patienten mit idiopathisch-generalisierter Epilepsie (ohne JME) ........................18

Tabelle 3: Patienten mit juveniler myoklonischer Epilepsie (JME) .....................................19

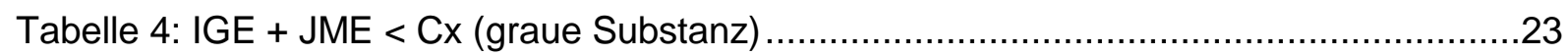

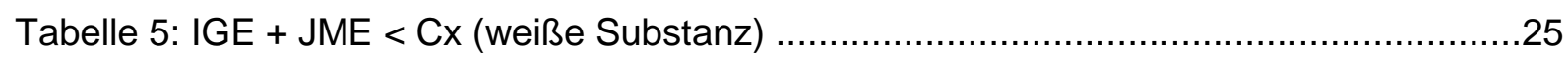

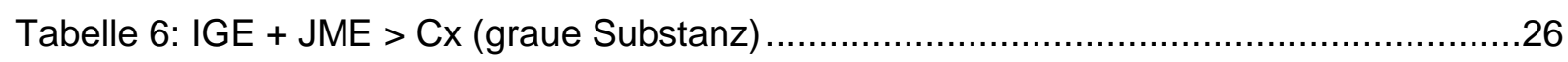

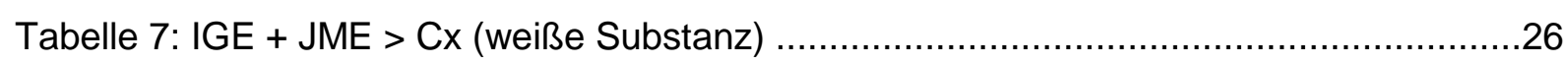

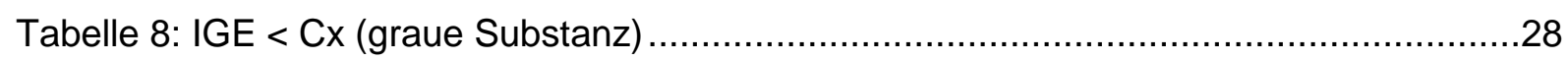

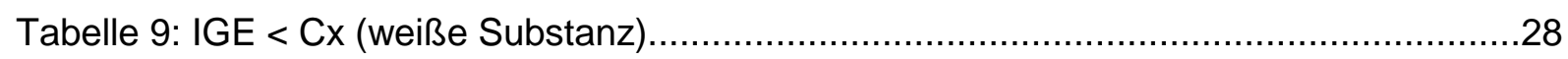

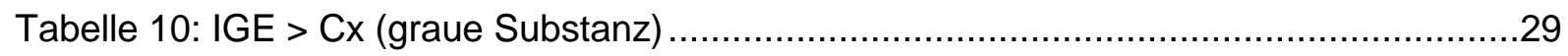

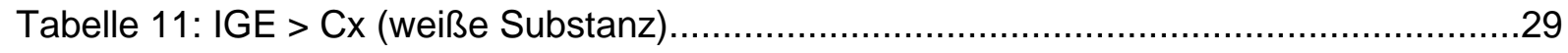

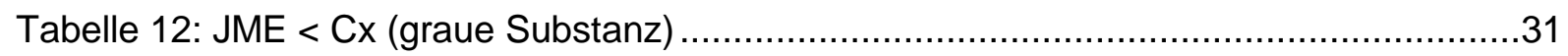

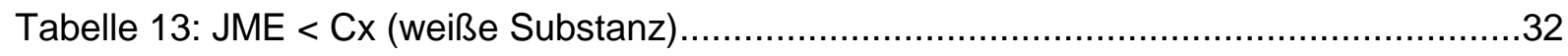

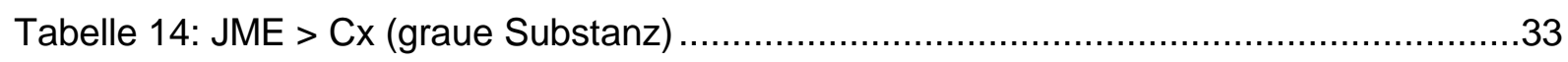

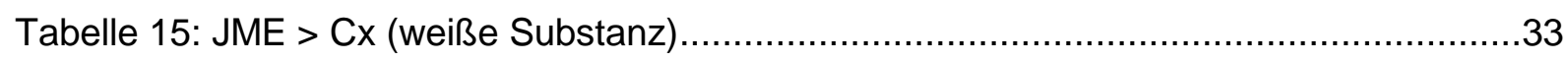

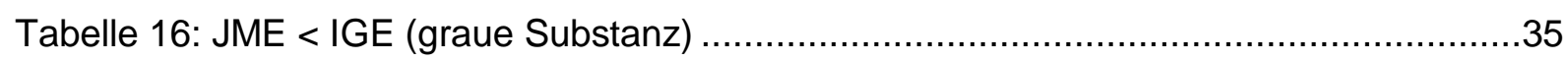

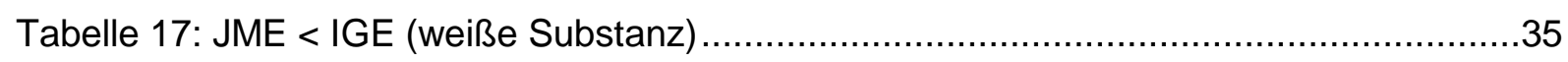

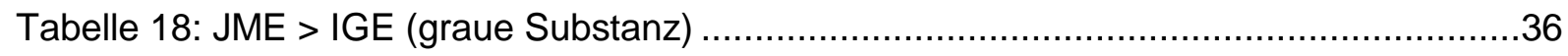

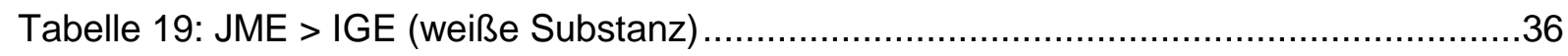

Tabelle 20: Gruppenvergleich (FA) von IGE und JME mit Cx .......................................

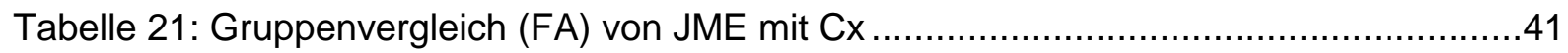

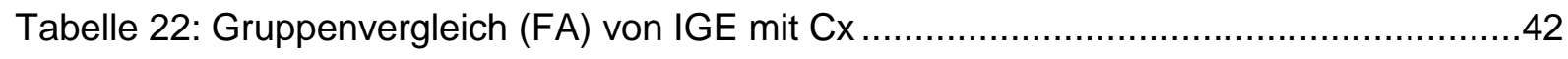

Tabelle 23: Gruppenvergleich (MD) von IGE und JME mit Cx ...................................43

Tabelle 24: Gruppenvergleich (MD) von JME mit Cx ...............................................43

Tabelle 25: Gruppenvergleich (MD) von IGE mit Cx .................................................44 


\section{$9 \quad$ Assoziierte Veröffentlichung}

Teile dieser Dissertation wurden im Rahmen des folgenden Artikels publiziert:

Focke NK, Diederich C, Helms G, Nitsche MA, Lerche H, Paulus W (2013): Idiopathicgeneralized epilepsy shows profound white matter diffusion-tensor imaging alterations. Hum Brain Mapp $\underline{35}$, 3333-3342 
10 Anhang

10.1 Fragebogen für die Untersuchung im MRT 
Fragebogen MR-Untersuchung

Fassung 3/2006

\section{Untersuchung: Magnetresonanz-Tomografie oder Spektroskopie}

MR-Forschung in der Neurologie und Psychiatrie

Bereich Humanmedizin - Georg-August-Universitat Gottingen

Name, Vorname:

Lesen Sie sich zu Ihrer eigenen Sicherheit diesen Fragebogen grundlich durch und beantworten Sie gewissenhaft alle Fragen. Wenn Sie sich nicht sicher sind oder eine Frage nicht verstehen, wenden Sie sich bitte an einen unserer Mitarbeiter. Unterschreiben Sie anschließend den Fragebogen und lassen Sie sich von einem Mitarbeiter eirweisen, bevor Sie den Magnet-Bereich betreten.

Wichtig. Aufgrund des sehr starken Magnetfeldes durfen keinerlei Gegenstande oder Gerate die aus Metall sind oder Metall enthalten könten, mit in den Untersuchungsraum genommen werden. Legen Sie solche Gegenstande und Gerate (z.B. Mobiltelefone, Manzen, Kugelschreiber, Schlüssel, Haarspangen, Uhren, Schmuck Brillen, Gurtel, Horgerate, Funkrufempfanger) unbedingt vorher abl

\section{Achtung: der Magnet ist immer an!}

Betreten Sie den Untersuchungsraum nur nach Aufforderung durch das Personal!

\section{Bitte Zutreffendes ankreuzen:}

\begin{tabular}{|c|c|c|}
\hline 1 & $\begin{array}{l}\text { Sind Sie Träger eines Herzschrittmachers, Defibrillators, Horgerats, Medikarnentenpumpe } \\
\text { (Insulin?). Neurostimulators, Implantat mit Magnetvertil (zB. kanstlicher Darmausgang)? } \\
\text { Wenn ja, welche? }\end{array}$ & 圂 Nein \\
\hline 2 & $\begin{array}{l}\text { Wurden Sie schon einmal an Kopf oder Herz operiert? } \\
\text { Wenn ja, warum? }\end{array}$ & 国 Nein \\
\hline 3 & $\begin{array}{l}\text { Befinden sich an oder auf threm Korper Metallteile oder metallhaltige Gerate (z. B. } \\
\text { Beinprothesen, Elektroden, Katheter, Langzeit-EKG, Bestrahlungsquelien, } \\
\text { Akupunkturnadeln, Plercing)? } \\
\text { Wenn ja, welche? }\end{array}$ & 四 Nein \\
\hline 4 & 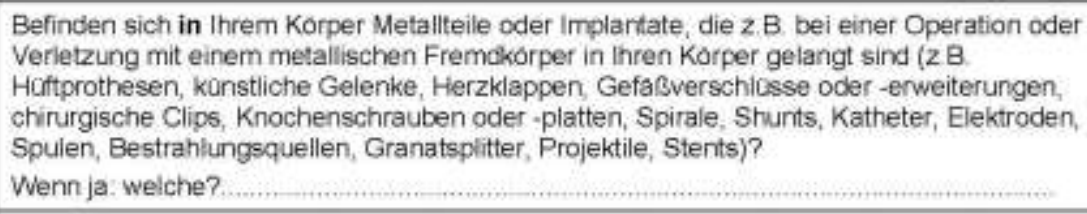 & 图 Nein \\
\hline 5 & Tragen Sie magnetisch fixierte Implantate ( $z$. B Zahnprothesen, Glasauge)? & (19) Nein \\
\hline 6 & Haben Sie beruflich oder privat mit der Verarbeitung von Metallen zu tun? & (19ain Nein \\
\hline 7 & $\begin{array}{l}\text { Tragen Sie (außer Amalgam-Fullungen) Zahnersatz, Brucken oder } \\
\text { Zahnklammern/-spangen? } \\
\text { Wenn ja: welche? }\end{array}$ & 10 Nein \\
\hline 8 & $\begin{array}{l}\text { Leiden Sie unter einer schweren Erkrankung der Atemwege, des Herz-Kreislaufsysterns } \\
\text { oder des Bewwegungssystems (z.B. Asthma, Herzschwache, Herzrhythmusstorungen, } \\
\text { Lahmungen)? } \\
\text { Wenn ja: welche? }\end{array}$ & (19) Nein \\
\hline 9 & Leiden Sie unter Diabetes oder einem Anfallsleiden (z.B. Epilepsie)? & Da Nein \\
\hline 10 & Neigen Sie zu Klaustrophobie, Schwindel- oder Panikanfallen? & 国 Neir \\
\hline 11 & Sind Sie tatowiert oder haben Sie ein permanentes Make-up? & (19) Nein \\
\hline
\end{tabular}




\begin{tabular}{|c|c|c|}
\hline 12 & Ist bei Ihnen eine Kontrastmittel-Allergie bekannt? & Na Nein \\
\hline 13 & $\begin{array}{l}\text { Leiden Sie unter anderen Allergien? } \\
\text { wenn ja, welche? }\end{array}$ & Ja Nein \\
\hline 14 & $\begin{array}{l}\text { Nehmen Sie zurzeit regeimaßig Medikamente ein? } \\
\text { wenn ja, welche? }\end{array}$ & Nein \\
\hline 15 & Haben Sie in den letzten 24 Stunden Medikamente oder Alkohol zu sich genommen? & 四 Nein \\
\hline 16 & Wurce in den letzten 3 Tagen eine MRT-Untersuchung durchgefahrt? & Ja Nein \\
\hline
\end{tabular}

Nur von Frauen auszufallen:

\begin{tabular}{|l|l|l|}
\hline 17 & Besteht die Moglichkeit, dass Sie schwanger sind? & 国 Nein \\
\hline 18 & Tragen Sie eine Kuplerspirale? & 国 Nein \\
\hline
\end{tabular}

Ich habe diese Information und alle mich betreffenden Fragen verstanden und nach bestem Wissen wahrheitsgemaß $B$ beantwortet.

Datum.

Unterschrift:

- wird vom Personal ausgefüllt -

$\square$ Untersuchung unbedenklich

$\square$ KEINE Untersuchung moglich

Bemerkungen:

Daturn:

Unterschritt 


\subsection{Aufklärung für die Studienteilnahme}




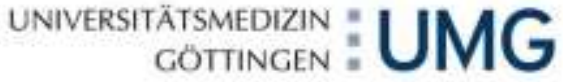

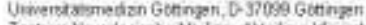

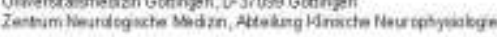

Herrn Max Mustermann

Nord-Süd-Str. 1

11111 Musterstadt

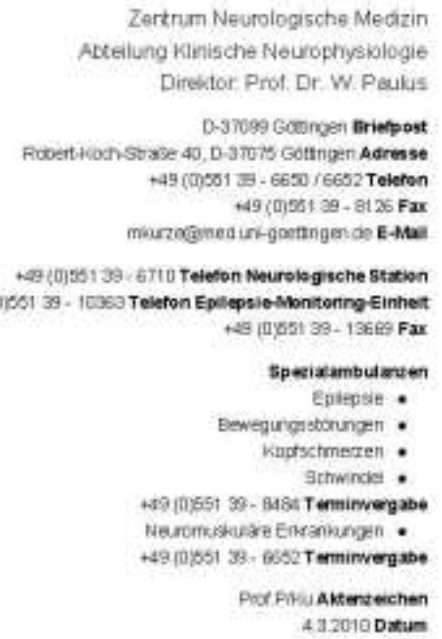

Sehr geehrter Herr Mustermann,

die Uniklinik Gottingen führt momentan eine klinische Studie zur Untersuchung von Epilepsie durch. Bei dieser Studie wird ein Kemspintomograph/ Magnetresonanztomograph, auch kurz MRT genannt, eingesetzt. Wie Sie vielleicht wissen, wird der Kemspintomograph zur Diagnostik von vielen verschiedenen Erkrankungen wie Morbus Parkinson. Epilepsie und anderen Erkrankungen eingesetzt. Mit dessen Hilfe konnen aber auch unterschiedliche Gewebe dargestellt und beurtelit werden. Es werden immer wieder neue Verfahren entwickeit mit denen man die verschiedenen Gewebe noch genauer darstelien und Erkrankungen noch schneller diagnostizieren kann. Das Zlel dieser Studie ist es, mit Hilfe neuer, innovativer Verfahren, die Diagnostik von Epilepsie zu verbessern.

Sie erfülen die Aufnahmekriterien für diese Studie und wir möchten Sie bitten, uns bei der Erforschung dieser wichtigen Erkrankung zu helfen. Eine Teilnahme an dieser Studie ist selbstverstandlich vollig freiwillig.

Die Untersuchung mit dem Kemspintomographen erfolgt ohne Röntgenstrahlung oder Radioaktivităt. Wăhrend der Untersuchung befindet sich ihr Korper in einem Magnetfeld. Radiowellenimpulse erzeugen Echosignale, die von empfindlichen Antennen aufgefangen werden Ein Computer errechnet hieraus Schnittbilder Ihres Köpers.

\section{Die Untersuchung:}

Die Untersuchung wird in einem speziellen Raum durchgefuhrt. Sie liegen auf einer Liege, die sich dann in die $65 \mathrm{~cm}$ große Offnung des Gerates bewegt. Die Untersuchung dauert ca. eine Stunde. Sie werden aber nur untersucht, wenn sie das typische Klopfen oder Pochen hören, das durch das Magnetfeld verursacht wird. Das Gerausch kann relativ laut sein, weshalb sie Kopfhörer zum Gehörschutz erhaiten. Außerdem haben Sie während der gesamten Untersuchung die Möglichkeit, 


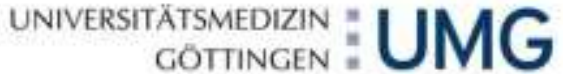

die durchfuhrende Assistentin zu kontaktieren. Von der Untersuchung selbst ist außer dem Klopfen im Normalfall nichts zu spüren.

Sie sollten während der Untersuchung ruhig und entspannt liegen und sich während der Untersuchung nicht bewegen und den Kopf ruhig halten. Falls es Ihnen nicht moglich ist, kónnen Sie jederzeit mit dem Untersucher uber eine Notfalklingel und eine Gegensprechanlage Kontakt aufnehmen.

\section{Sicherheit:}

Aufgrund des starken Magnetfeldes, das auch außerhalb der Untersuchung besteht, dürfen keine Gegenstande oder Gerate, die aus Metall sind oder Metall enthalten, mit in den Untersuchungsraum genommen werden. Bitte legen Sie Mobiltelefone, Münzen, Kugelschreiber. Haarspangen, Uhren. Schmuck, Brillen, Gurtel usw, vorher ab.

Die Untersuchung selbst ist ein völlig ungefăhrliches Verfahren. Für Personen mit Metallclips oder einem Herzschrittmacher konnen jedoch erhebliche Gefahren durch das Magnetfeld entstehen. So kőnnen durch das Magnetfeld Knochenschrauben verdreht und Gefăßclips gelöst werden oder der Herzschrittmacher außer Kraft gesetzt werden. Damit wir eine Gefährdung ausschließen können, erhalten Sie von uns einen Fragebogen, den Sie bitte grundlich durchlesen und gewissenhaft ausfülen.

Wenn Sie sich entscheiden an dieser Studie teilzunehmen, wärden wir uns bemühen einen passenden Termin für die Untersuchung im MRT zu finden z.B. wäre eine Kombination von MRTUntersuchung mit einer bereits geplanten Wiedervorstellung in der Ambulanz denkbar. Neben der MRT-Untersuchung benötigen wir auch noch einige Angaben uber litre Erkrankung, die wir aus Ihrer Krankenakte entnehmen. Alle erhobenen Daten unterliegen selbstverstandlich der arztlichen Schweigepflicht und werden im Rahmen der Studie verschlüsselt.

Durch die Teilnahme an dieser Studie entstehen Ihnen weder Kosten noch profitieren sie direkt davon, da diese Studie wissenschaftlichen Zwecken dient. Allerdings könnten Sie anderen helfen, dass ihre Krankheit früzeitig erkannt und schnellstmoglich einer entsprechenden Therapie zugefuhrt wird. Die weitere Behandlung Ihrer Erkrankung wird durch die Teilnahme an der Studie nicht beeinflusst. Als Anerkennung fur Ihren Aufwand können wir thnen eine Entschadigung in Hohe von 20 Euro anbieten.

Ich bedanke mich im Voraus fur Ihre Bereitschaft, an dieser Studie teilzunehmen. Sie stellen sich in den Dienst der Wissenschaft und helfen vielen Epilepsie-Patienten.

Mit freundichen Grußen

$\begin{array}{lll}\text { Dr. Niels Focke } & \text { Dr. Vanda Edit Toth } & \text { Christine Diederich } \\ \text { (Arzt) } & \text { (Stipendiatin) } & \text { (Studentin) }\end{array}$


10.3 Einwilligung in die Studienteilnahme 


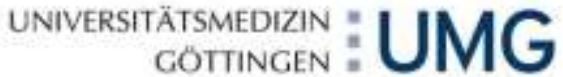

Uewerstsumadien Gutmen, a sroge Gotmzen

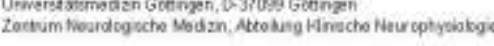

Herm Max Mustermann Nord-Sud-Str. 1

11111 Musterstadt
Zentrum Neurologische Medizin Atkeilung Klinische Neurophysiologie Direstor Prot. Dr W. Paukis

D-3 Fobert tioch swase 43, D-37075 Gottingen Adresse 49 rossisi $139-6650$ i 6062 Teleton

+48 (0) 000139 - का 26 Fax Iniurzogmetion-goetungen de E-Ma:

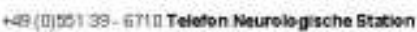

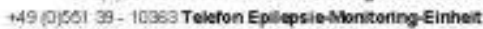

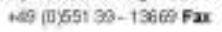

Spetixambuarten

Eplepsir -

Bewegungrationugran :

Kaproctmerzen -

Scrwinas -

49 10p51 38- BuE4 Terminvergabe

Nesumustalare Enrankungen -

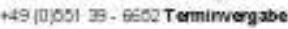

Pror Piociakswewethen

432010 Datum

Ich willige ein, an der Studie:

Einwilligung

"Multi-Parameter Mapping bel fokaler und generalisierter Epilepsie“

der Uniklinik Gottingen teilzunehmen. Den Aufklärungsbogen habe ich gelesen und verstanden.

Datenschutzerklärung:

lch bin damit einverstanden, dass meine persónlichen Daten elektronisch und auf Papier gespeichert werden. Daruber hinaus gestatte ich, den an dieser Studie beteiligten Arzten unter Wahrnehmung der ärztlichen Schweigejdlicht. Einblick in meine Krankenakte zu nehmen. Die. Daten werden von den Prufärzten zur wissenschaftlichen Auswertung der Studie verwendet

Sie können die erteilte Einwilligung in die Studienteilnahme jederzeit ohne Angabe von Gründen widerrufen:

Sollte im Rahmen der Studie ein Zufallsbefund (krankhafte Verănderung des Gehims, die zuvor nicht bekannt war) entdeckt werden, so mochte ich:

- dass ich hieruber informiert werde.

- dass mein Arzt hierüber informiert wird.

- dass weder ich noch mein behandelnder Arzt hierüber informiert wird.

पत्रापणm

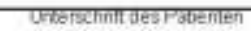




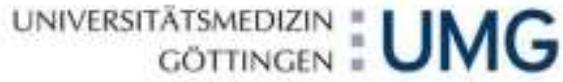

Bitte senden Sie die Einwilligung an die Uniklinik Gottingen mit beiliegendem Freiumschlag zurück.

Damit wir die MRT-Untersuchung mit ihrem nächsten Ambulanztermin verbinden können. benơtigen wir noch das Datum ihres năchsten Ambulanztermins:

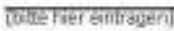

Um Ihnen eventuell kurzfristige Terminänderungen mitzuteilen, benotigen wir noch die Angabe einer Telefonnummer oder einer E-Mail-Adresse, unter der wir Sie erreichen können. Die Angabe ist selbstverstandlich freiwillig:

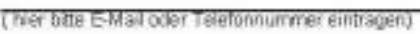

Wir bedanken uns im Voraus für Ihre Bereitschaft an der Studie teilzunehmen. Falls Sie noch Fragen haben, können Sie sich geme an Dr. Focke (Arzt, Abteilung Neurologie E-Mail: nfockeouni-goettingen de) oder an Christine Diederich (Studentin und Doktorandin: Tel:: 0163 6217821 o. E-Mail: c.diederichos stud uni-ooettingen.de) und Vanda Toth (Studentin und Doktorandin; E-Mail: vandaedit@gmail.com oder Tel: 0551-39-12310) wenden.

Mit freundlichen Grußen

Dr. N. Focke

(Arat)

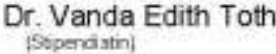

Christine Diederich \{Studentin' 


\section{Danksagung}

Mein besonderer Dank gilt Prof. Dr. Walter Paulus sowie Dr. Niels Focke für die Ermöglichung dieser Arbeit in der Klinik für Klinische Neurophysiologie. Für die engagierte und persönliche Betreuung von Dr. Niels Focke möchte ich mich herzlich bedanken. Seine praktischen Ratschläge und fachlichen Hinweise waren mir eine große Unterstützung.

Des Weiteren gilt mein Dank den Mitarbeiterinnen und Mitarbeitern der Abteilung für MRForschung in der Neurologie und Psychiatrie der UMG: Ilona Pfahlert, Britta Perl, Nina Kaeding und Dr. Gunther Helms. Sie waren mir bei der Durchführung der MR-Untersuchung sowie bei fachlichen Fragestellungen eine große und wertvolle Hilfe.

Ebenso möchte ich den Mitarbeiterinnen der Abteilung für Klinische Neurophysiologie für ihre tatkräftige Mithilfe bei der Organisation der Patientenkontakte danken. 